\title{
EXISTENCE THEOREMS FOR WEAK AND USUAL OPTIMAL SOLUTIONS IN LAGRANGE PROBLEMS WITH UNILATERAL CONSTRAINTS. I $\left({ }^{1}\right)$
}

\author{
BY \\ LAMBERTO CESARI
}

\section{Contents of Part I.}

1. The problem, p. 371

2. The space of continuous vector functions, p. 371

3. Admissible pairs $u(t), x(t)$, p. 372

4. Upper semicontinuity of variable sets, p. 372

5. Closure theorems, p. 382

6. Notations for Lagrange problems with unilateral constraints, p. 389

7. An existence theorem for Lagrange problems with unilateral constraints, p. 390

8. Example of a problem with no absolute minimum, p. 398

9. Another existence theorem for Lagrange problems with unilateral constraints, p. 400

10. Examples, p. 401

11. The free problems, p. 403

12. Lagrange problems with $f$ linear in $u$, p. 404

13. Existence theorems for Lagrange problems with $f$ linear in $u$, p. 409

\section{Contents of Part II.}

14. Weak solutions, p. 414

15. Sufficient conditions for property $(P)$, p. 417

16. Existence theorems for weak solutions, p. 427

Introduction. In the present papers (I and II) we prove existence theorems for weak and usual optimal solutions of nonparametric Lagrange problems with (or without) unilateral constraints.

We consider arbitrary pairs $x(t), u(t)$ of vector functions, $u(t)$ measurable with values in $E_{m}, x(t)$ absolutely continuous with values in $E_{n}$, and we discuss the existence of the absolute minimum of a functional

$$
I[x, u]=\int_{t_{1}}^{t_{2}} f_{0}(t, x(t), u(t)) d t,
$$

Received by the editors December 6, 1965.

(1) Research partially supported by NSF grant GP-3920 at The University of Michigan. 
with side conditions represented by a differential system

$$
d x / d t=f(t, x(t), u(t)), \quad t_{1} \leqq t \leqq t_{2},
$$

constraints

$$
(t, x(t)) \in A, u(t) \in U(t, x(t)), \quad t_{1} \leqq t \leqq t_{2},
$$

and boundary conditions

$$
\left(t_{1}, x\left(t_{1}\right), t_{2}, x\left(t_{2}\right)\right) \in B,
$$

where $A$ is a given closed subset of the $t x$-space $E_{1} \times E_{n}$, where $B$ is a given closed subset of the $t_{1} x_{1} t_{2} x_{2}$-space $E_{2 n+2}$, and where $U(t, x)$ denotes a given closed variable subset of the $u$-space $E_{m}$, depending on time $t$ and space $x$. Here $A$ may coincide with the whole space $E_{1} \times E_{n}$, and $U$ may be fixed and coincide with the whole space $E_{m}$.

In the particular situation, where the space $U$ is compact for every $(t, x)$, these problems reduce to Pontryagin's problems; in the particular situation where the space $U$ is fixed and coincides with the whole space $E_{m}$, then these problems have essentially the same generality of usual Lagrange problems. Throughout these papers we shall assume $U(t, x)$ to be any closed subset of $E_{m}$.

In $\S \S 1-5$ we prove closure theorems for usual solutions. In $\S \S 6-12$ we prove existence theorems for usual solutions. These contain as particular cases the Filippov existence theorem for Pontryagin's problems $(U(t, x)$ compact), existence theorems for usual Lagrange problems $\left(U=E_{m}\right)$, and the Nagumo-Tonelli existence theorem for free problems $(m=n, f=u)$. In Part II we prove existence theorems for weak (or generalized) solutions introduced as measurable probability distributions of usual solutions (Gamkrelidze chattering states).

In subsequent papers we shall extend some of the present results to multidimensional Lagrange problems involving partial differential equations in Sobolev spaces with unilateral constraints.

We begin with an analysis of the concept of upper semicontinuity of variable subsets in $E_{m}$. The usual concept of upper semicontinuity is replaced by two others (properties $(U)$ and $(Q), \S 4)$, which are essentially more general than upper semicontinuity, in the sense that closed sets $U(t, x)$, for which upper semicontinuity property holds, certainly satisfy property $(U)$, and closed and convex sets $Q(t, x)$, for which upper semicontinuity property holds, certainly satisfy property $(Q)$. In $(\$ 5)$ we then extend the closure theorem of A. F. Filippov in various ways, so as to include, among other things, the use of pointwise and not necessarily uniform convergence of some components of a sequence of trajectories. In $\S \S 7,9$ we prove existence theorems for optimal usual solutions by a new analysis of a minimizing sequence, and by using the above extensions of Filippov's closure theorem. In $\S \S 11,13$ we then deduce existence theorems for the case where $f$ is linear in $u$, 
and for free problems of the calculus of variations $(m=n, f=u)$. Finally, in Part II, §16, we prove existence theorems for weak solutions in the general case above, as well as for the case in which $f$ is linear, and for free problems.

1. The problem. We denote by $x$ a variable $n$-vector $x=\left(x^{1}, \cdots, x^{n}\right) \in E_{n}$, by $u$ a variable $m$-vector $u=\left(u^{1}, \cdots, u^{m}\right) \in E_{m}$, and by $t \in E_{1}$ the independent variable. We denote by $A$ an arbitrary subset of the $(t, x)$-space, $A \subset E_{1} \times E_{n}$, and, for any $(t, x) \in A$, we denote by $U=U(t, x)$ a variable subset of the $u$-space, $U(t, x) \subset E_{m}$. In the terminology of control problems, $u$ is the control variable and $U(t, x)$ the control space. We denote by $f_{i}(t, x, u), i=0,1, \cdots, n$, given real functions defined for all $(t, x) \in A$, and all $u \in U(t, x)$, and by $f$ the $n$-vector function $f=\left(f_{1}, \cdots, f_{n}\right)$. We denote by $B$ a given subset of the $(2 n+2)$-space $\left(t_{1}, x_{1}, t_{2}, x_{2}\right)$. We are interested in the determination of a measurable vector function $u(t), t_{1} \leqq t \leqq t_{2}$, (control function, or steering function, or strategy), and a corresponding absolute continuous vector function $x(t), t_{1} \leqq t \leqq t_{2}$, (trajectory), satisfying almost everywhere the differential system

$$
d x / d t=f(t, x(t), u(t)), \quad t_{1} \leqq t \leqq t_{2},
$$

satisfying the boundary conditions

$$
\left(t_{1}, x\left(t_{1}\right), t_{2}, x\left(t_{2}\right)\right) \in B,
$$

satisfying the constraints

$$
\begin{aligned}
(t, x(t)) \in A, & t_{1} \leqq t \leqq t_{2}, \\
u(t) \in U(t, x(t)), & \text { a.e. in }\left[t_{1}, t_{2}\right],
\end{aligned}
$$

and for which the integral (cost functional)

$$
I[x, u]=\int_{t_{1}}^{t_{2}} f_{0}(t, x(t), u(t)) d t
$$

has its minimum value (see $\S \S 2,3$ for details). We shall assume that $U(t, x)$ is closed for every $(t, x) \in A$.

2. The space of continuous vector functions. Let $X$ be the collection of all continuous $n$-dim vector functions $x(t)$ defined on arbitrary finite intervals of the $t$-axis:

$$
x(t)=\left(x^{1}, \cdots, x^{n}\right), \quad a \leqq t \leqq b, \quad x(t) \in E_{n},
$$

If $x(t), a \leqq t \leqq b$, and $y(t), c \leqq t \leqq d$, are any two elements of $X$, we shall define a distance $\rho(x, y)$. First, let us extend $x(t)$ and $y(t)$ outside their intervals of definition by constancy and continuity in $(-\infty,+\infty)$, and then let

$$
\rho(x, y)=|a-c|+|b-d|+\max |x(t)-y(t)|,
$$

where max is taken in $(-\infty,+\infty)$. It is known that $X$ is a complete metric 
space when equipped with the metric $\rho$. Ascoli's theorem can now be expressed by saying that any sequence of equicontinuous vector functions $x_{n}$ of $X$, whose graphs in the $t x$-space are equibounded, possesses at least one subsequence which is convergent in the $\rho$-metric toward an element $x$ of $X$.

3. Admissible pairs $u(t), x(t)$. Let $A$ be a closed subset of the $(t, x)$-space $E_{1} \times E_{n}$. For every $(t, x) \in A$ let $U(t, x)$, or control space, be a subset of the $u$-space $E_{m}$. Let $M$ be the set of all $(t, x, u)$ with $(t, x) \in A, u \in U(t, x)$. Let $f(t, x, u)=\left(f_{1}, \cdots, f_{n}\right)$ be a continuous vector function defined on $M$. We shall denote by $Q(t, x)$ the set of all values in $E_{n}$ taken by $f(t, x, u)$ when $u$ describes $U(t, x)$, or $Q(t, x)$ $=f(t, x, U(t, x))$. A vector function $u(t)=\left(u^{1}, \cdots, u^{m}\right), t_{1} \leqq t \leqq t_{2}$ (control function) and a vector function $x(t)=\left(x^{1}, \cdots, x^{n}\right), t_{1} \leqq t \leqq t_{2}$ (trajectory) are said to be an admissible pair provided (a) $u(t)$ is measurable in $\left[t_{1}, t_{2}\right]$; (b) $x(t)$ is absolutely continuous (AC) in $\left[t_{1}, t_{2}\right]$; (c) $(t, x(t)) \in A$ for every $t \in\left[t_{1}, t_{2}\right]$; (d) $u(t) \in U(t, x(t))$ a.e. in $\left[t_{1}, t_{2}\right]$; (e) $d x / d t=f(t, x(t), u(t))$ a.e. in $\left[t_{1}, t_{2}\right]$. By the expression the vector function $x(t), t_{1} \leqq t \leqq t_{2}$, is a trajectory, we shall mean below that there exists a vector function $u(t), t_{1} \leqq t \leqq t_{2}$, such that the pair $u(t), x(t)$ satisfies (a)-(e). We say also that $x(t)$ is generated by $u(t)$.

4. Upper semicontinuity of variable sets. In view of using sets $U(t, x), Q(t, x)$ which are closed but not necessarily compact, we need a concept of upper semicontinuity which is essentially more general than the usual one. We shall introduce two modifications of the usual definition of upper semicontinuity, and we shall denote them as "property $(U)$ ", and "property $(Q)$ ", since we shall usually use them for the sets $U(t, x)$ and $Q(t, x)$ above, respectively.

We shall discuss properties $(U)$ and $(Q)$ first in relation to arbitrary variable sets $U(t, x), Q(t, x)$ which are functions of $(t, x)$ in $A$. Then we shall discuss their relations when $Q(t, x)$ is assumed to be the image of $U(t, x)$ as mentioned in $\S 3$. Properties proved for $U(t, x)$ under conditions $(U)$ or $(Q)$, will be used for $Q(t, x)$ when this set satisfies conditions $(U)$ or $(Q)$.

(A) The property $(U)$. Given any set $F$ in a linear space $E$ we shall denote by $\operatorname{cl} F, \operatorname{co} F$, bd $F$, int $F$ respectively the closure of $F$, the convex hull of $F$, the boundary of $F$, the set of all interior points of $F$. Thus, clco $F$ denotes the closure of the convex hull of $F$. We know that $F, \operatorname{cl} F, \operatorname{co} F, \operatorname{cocl} F$ are all contained in clco $F$.

For every $(t, x) \in A$ and $\delta>0$ let $N_{\delta}(t, x)$ denote the closed $\delta$-neighborhood of $(t, x)$ in $A$, that is, the set of all $\left(t^{\prime}, x^{\prime}\right) \in A$ at a distance $\leqq \delta$ from $(t, x)$.

A variable subset $U(t, x),(t, x) \in A$, is said to be an upper semicontinuous function of $(t, x)$ at the point $(\bar{t}, \bar{x}) \in A$ provided, given $\varepsilon>0$, there is a number $\delta=\delta(\bar{t}, \bar{x}, \varepsilon)>0$ such that $(t, x) \in N_{\delta}(\bar{t}, \bar{x})$ implies $U(t, x) \subset[U(\bar{t}, \bar{x})]_{\varepsilon}$, where $[U]_{\varepsilon}$ denotes the closed $\varepsilon$-neighborhood of $U$ in $E_{m}$.

Again, let $U(t, x),(t, x) \in A, U(t, x) \subset E_{m}$, be a variable subset of $E_{m}$, which is a function of $(t, x)$ in $A$. For every $\delta>0$ let $U(t, x, \delta)=\bigcup U\left(t^{\prime}, x^{\prime}\right)$, where the 
union is taken for all $\left(t^{\prime}, x^{\prime}\right) \in N_{\delta}(t, x)$. We shall say that $U(t, x)$ has property $(U)$ at $(\bar{t}, \bar{x})$ in $A$, if

$$
U(\bar{t}, \bar{x})=\bigcap_{\delta>0} \operatorname{cl} U(\bar{t}, \bar{x}, \delta) .
$$

We shall say that $U(t, x)$ has property $(U)$ in $A$, if $U(t, x)$ has property $(U)$ at every $(t, x)$ of $A$.

(i) If $U(t, x)$ has property $(U)$ at $(\bar{t}, \bar{x})$, then $U(\bar{t}, \bar{x})$, being the intersection of closed sets, is closed.

(ii) If $A$ is closed, and $U(t, x)$ is any variable set $M$ which is a function of $(t, x)$ in $A$ and has property $(U)$ in $A$, then the set $M$ of all $(t, x, u) \in A \times E_{m}$ with $u \in U(t, x)$, $(t, x) \in A$, is closed.

Proof. If $(\bar{t}, \bar{x}, \bar{u}) \in \operatorname{cl} M$ and $\varepsilon>0$, then there are $\infty$-many points $(t, x, u) \in M$ with $|t-\bar{t}|<\varepsilon,|x-\bar{x}|<\varepsilon,|u-\bar{u}|<\varepsilon$. Thus, $(\bar{t}, \bar{x}) \in A$ since $A$ is closed, $(t, x) \in N_{2 \varepsilon}(\bar{t}, \bar{x}), \quad u \in U(t, x), \quad u \in U(\bar{t}, \bar{x}, 2 \varepsilon), \quad$ and $\bar{u} \in \bigcap_{\varepsilon} \operatorname{cl} U(\bar{t}, \bar{x}, 2 \varepsilon)=U(\bar{t}, \bar{x})$, $\bar{u} \in U(\bar{t}, \bar{x})$, since $U$ has property $(U)$ at $(\bar{t}, \bar{x})$. This proves that $(\bar{t}, \bar{x}, \bar{u}) \in M$, that is, $M$ is closed.

Note that the sets $U(\bar{t}, \bar{x}, \delta)$ are not necessarily closed even if $A$ is closed, all sets $U(t, x)$ are closed, and we take for $N_{\delta}(\bar{t}, \bar{x})$ the closed $\delta$-neighborhood if $(\bar{t}, \bar{x})$ is in $A$ as stated. This can be seen by the following example. Let $A=[0 \leqq t \leqq 1,0 \leqq x \leqq 1]$ a subset of $E_{2}$, and

$$
U(t, x)=\left[z=\left(z_{1}, z_{2}\right) \mid z_{2} \geqq t z_{1},-\infty<z_{1}<+\infty\right]
$$

for $0<t \leqq 1$, and $U(0, x)=\left[z_{2} \geqq 0, z_{1}=0\right]$ for $t=0$. Then $U(0, x, \delta)$ $=\left[z=\left(z_{1}, z_{2}\right) \mid z_{2} \geqq \delta z_{1}\right.$ for $-\infty<z_{1} \leqq 0$, and $z_{2}>0$ for $\left.0<z_{1}<\infty\right]$ for any $\delta>0$. The sets $U(0, x, \delta)$ are not closed. Here $U(t, x)$ does not satisfy property $(U)$ at the points $(0, x)$. Nevertheless, the statement holds:

(iii) If $A$ is closed, and $U(t, x)$ satisfies property $(U)$ in $A$, then the sets $U(t, x, \delta),(t, x) \in A, \delta>0$, are all closed.

Proof. Let $M_{\delta}$ denote the set of all points $(t, x, u)$ with $(t, x) \in N_{\delta}(\bar{t}, \bar{x}), u \in U(t, x)$. Obviously $N_{\delta}(\bar{t}, \bar{x}) \subset A \subset E_{n+1} ; M_{\delta} \subset E_{n+1} \times E_{m}$, and $N_{\delta}(\bar{t}, \bar{x})$ is compact and $M_{\delta}$ is closed by force of (ii) above. Let $\bar{u}$ be a point of accumulation of $U(t, x, \delta)$, and for any $\eta>0$ let $V_{\eta}(\bar{u})$ denote the $\eta$-neighborhood of $\bar{u}$ in $E_{m}$. Then $M_{\delta} \cap\left(V_{\eta}(\bar{u}) \times E_{n+1}\right) \subset N_{\delta}(\bar{t}, \bar{x}) \times V_{\eta}(\bar{u})$, hence $M_{\delta} \cap\left(V_{\eta}(\bar{u}) \times E_{n+1}\right)$ is bounded. Since both $M_{\delta}$ and $V_{\eta}(\bar{u}) \times E_{n+1}$ are closed sets, the set $M_{\delta} \cap\left(V_{\eta}(\bar{u}) \times E_{n+1}\right)$ is closed and bounded, and therefore a compact subset of $E_{n+1} \times E_{m}$. Now the set $U(\bar{t}, \bar{x}, \delta) \cap V_{\eta}(\bar{u})$ is the projection of $M_{\delta} \cap\left(V_{\eta}(\bar{u}) \times E_{n+1}\right)$ on the $u$-space $E_{m}$, and therefore $U(\bar{t}, \bar{x}, \delta) \cap V_{\eta}(\bar{u})$ is compact. Thus $\bar{u} \in U(\bar{t}, \bar{x}, \delta) \cap V_{\eta}(\bar{u})$, and finally $\bar{u} \in U(\bar{t}, \bar{x}, \delta)$. Thus, $U(\bar{t}, \bar{x}, \delta)$ is closed, or $\operatorname{cl} U(\bar{t}, \bar{x}, \delta)=U(\bar{t}, \bar{x}, \delta)$, and $U(\bar{t}, \bar{x})$ $=\bigcap_{\delta} \operatorname{cl} U(\bar{t}, \bar{x}, \delta)=\bigcap_{\delta} U(\bar{t}, \bar{x}, \delta)$.

(iv) If $A$ is closed and $U_{j}(t, x),(t, x) \in A, j=1, \cdots, v, v$ finite, are variable 
subsets of $E_{m}$ all satisfying property $(U)$ in $A$, then their union and their intersection $V(t, x)=\bigcup_{j} U_{j}(t, x), W(t, x)=\bigcap_{j} U_{j}(t, x),(t, x) \in A$, are subsets of $E_{m}$ satisfying property $(U)$ in $A$. The same holds for their product $V(t, x)=U_{1} \times \cdots \times U_{v}$.

The proof is straightforward.

Under the hypotheses of (ii) the set $M$ is closed but not necessarily compact as the trivial example $U(t, x)=E_{m}, M=A \times E_{m}$, shows. The set $M$ is closed but not necessarily compact even if we assume that $A$ is compact, and that every $U(t, x)$ is compact. This is proved by the following example. Let $m=n=1$, $A=\left[(t, x) \in E_{2} \mid 0 \leqq t \leqq 1, \quad 0 \leqq x \leqq 1\right], \quad U(0, x)=\left[u \in E_{1} \mid 0 \leqq u \leqq 1\right]$, and, if $t \neq 0, U(t, x)=\left[u \in E_{1} \mid 0 \leqq u \leqq 1\right.$, and $\left.u=t^{-1}\right]$. Then $M$ is the set of all $(t, x, u)$ with $0 \leqq t \leqq 1,0 \leqq x \leqq 1$, and $0 \leqq u \leqq 1$, or $u=t^{-1}$ if $t \neq 0$. Obviously, $M$ is closed but not compact. Nevertheless, the statement holds:

(v) If $A$ is compact, if the variable set $U(t, x)$ is compact and convex for every $(t, x) \in A$ and possess property $(U)$ in $A$, if for every $(t, x) \in A$ there is some $\delta=\delta(t, x)>0$ such that $U(t, x) \cap U\left(t^{\prime}, x^{\prime}\right) \neq \varnothing$ for every $\left(t^{\prime}, x^{\prime}\right) \in N_{\delta}(t, x)$, then $M$ is compact.

Proof. If $M$ is not compact, then there is some sequence of elements $\left(t_{k}, x_{k}, u_{k}\right) \in M, k=1,2, \cdots$, with $\left(t_{k}, x_{k}\right) \in A,\left|t_{k}\right|+\left|x_{k}\right|+\left|u_{k}\right| \rightarrow+\infty$. Since $A$ is compact and hence bounded, we have $\left|u_{k}\right| \rightarrow+\infty$. On the other hand, there is some subsequence, say still $\left(t_{k}, x_{k}\right)$, with $t_{k} \rightarrow \bar{t}, x_{k} \rightarrow \bar{x},(\bar{t}, \bar{x}) \in A$. Given $\varepsilon>0$, we have $u_{k} \in U(\bar{t}, \bar{x}, \varepsilon)$ for all $k$ sufficiently large, as well as $U(\bar{t}, \bar{x}) \cap U\left(t_{k}, x_{k}\right) \neq \varnothing$. Since $U(\bar{t}, \bar{x})$ is compact, there is a solid sphere $S$ containing all of $U(\bar{t}, \bar{x})$ in its interior, say $U(\bar{t}, \bar{x}) \subset$ int $S \subset E_{m}$. On the other hand, if $\bar{u}_{k} \in U(\bar{t}, \bar{x}) \cap U\left(t_{k}, x_{k}\right)$, we have $\bar{u}_{k} \in$ int $S$, and $u_{k} \in E_{m}-S$, again for $k$ large. Since both $\bar{u}_{k}$ and $u_{k}$ belong to the convex set $U\left(t_{k}, x_{k}\right)$, the segment $\bar{u}_{k} u_{k}$ is contained in $U\left(t_{k}, x_{k}\right)$. In particular, if $u_{k}^{\prime}$ is the point where the segment $\bar{u}_{k} u_{k}$ intersects bd $S$, we have $u_{k}^{\prime} \in U\left(t_{k}, x_{k}\right)$, $u_{k}^{\prime} \in U(\bar{t}, \bar{x}, \varepsilon)$, and $u_{k}^{\prime} \in \operatorname{bd} S$. If $u^{\prime}$ is any point of accumulation of $\left[u_{k}^{\prime}\right]$, then $u^{\prime} \in \operatorname{bd} S$, and $u^{\prime} \in \operatorname{cl} U(\bar{t}, \bar{x}, \varepsilon)$ for ever $y \varepsilon>0$. Hence, $u^{\prime} \in \bigcap_{\varepsilon} \operatorname{cl} U(\bar{t}, \bar{x}, \varepsilon)=U(\bar{t}, \bar{x})$, a contradiction, since $U(\bar{t}, \bar{x}) \subset$ int $S$. We have proved that $M$ is compact.

(vi) If the set $U(t, x)$ is closed for every $(t, x) \in A$ and is an upper semicontinuous function of $(t, x)$ in $A$, then $U(t, x)$ has property $(U)$ in $A$.

Proof. By hypothesis $U(t, x, \delta) \subset[U(t, x)]_{\varepsilon}$, where $U_{\varepsilon}$ is closed. Hence $\operatorname{cl} U(t, x, \delta) \subset[U(t, x)]_{\varepsilon}$ for $\delta=\delta(t, x, \varepsilon)$ and any $\varepsilon>0$. Since $U(t, x)$ is closed, then $[U(t, x)]_{\varepsilon} \rightarrow U(t, x)$ as $\varepsilon \rightarrow 0+$. Thus $\bigcap_{\delta} \operatorname{cl} U(t, x, \delta) \subset U(t, x)$. Since the opposite inclusion is trivial, we have $\bigcap_{\delta} \operatorname{cl} U(t, x, \delta)=U(t, x)$. Statement (vi) is thereby proved.

The upper semicontinuity property implies property $(U)$, but the converse is not true, that is, the upper semicontinuity property for closed sets is more restrictive than property $(U)$. This is shown by the example after statement (iv) above in which all sets are closed. Another example is as follows. Take $n=2$ and

$$
U(t, x)=\left[\left(u^{1}, u^{2}\right) \in E_{2} \mid 0 \leqq u^{1}<+\infty, 0 \leqq u^{2} \leqq t u^{1}\right]
$$


for every $(t, x) \in A=\left[(t, x) \in E_{2} \mid 0 \leqq t \leqq 1,0 \leqq x \leqq 1\right]$. Then, for $\delta>0$, we have

$$
U(t, x, \delta)=\left[\left(u^{1}, u^{2}\right) \in E_{2} \mid 0 \leqq u^{1}<+\infty, 0 \leqq u^{2} \leqq(t+\delta) u^{1}\right],
$$

hence $U(t, x)=\bigcap_{\delta} \operatorname{cl} U(t, x, \delta)$ and $U(t, x)$ has property $(U)$ in $A$. On the other hand,

$$
[U(t, x)]_{\varepsilon}=\left[\left(u^{1}, u^{2}\right) \in E_{2} \mid 0 \leqq u^{1}<+\infty,-\varepsilon \leqq u^{2} \leqq t u^{1}+\varepsilon\left(1+t^{2}\right)^{1 / 2}\right] \cup N_{1},
$$

where $N_{1}=N_{\varepsilon}(0,0)=\left[\left(u^{1}, u^{2}\right) \mid\left(u^{1}\right)^{2}+\left(u^{2}\right)^{2} \leqq \varepsilon^{2}\right]$ if $t=0$, and, if $t \neq 0$,

$$
N_{1}=N_{\varepsilon}(0,0) \cup\left[\left(u^{1}, u^{2}\right) \in E_{2} \mid u^{1} \leqq 0, u^{2} \geqq-t^{-1} u^{1},-t u^{1}+u^{2} \leqq \varepsilon\left(1+t^{2}\right)^{1 / 2}\right] .
$$

Obviously $U\left(t^{\prime}, x^{\prime}\right)-[U(t, x)]_{\varepsilon} \neq \varnothing$ for $t^{\prime}>t$, hence $U(t, x)$ is not an upper semicontinuous function of $(t, x)$.

(vii) If $A$ is compact, if $U(t, x)$ is compact for every $(t, x) \in A$ and is an upper semicontinuous function of $(t, x)$ in $A$, then $M$ is compact.

(viii) If $A$ is closed and $U_{j}(t, x),(t, x) \in A, j=1, \cdots, v, v$ finite, are variable subsets of $E_{m}$ all upper semicontinuous functions of $(t, x)$ in $A$, then their union $V(t, x)$ and their intersection $W(t, x)$ are semicontinuous functions of $(t, x)$ in $A$. The same holds for their product $V(t, x)=U_{1} \times \cdots \times U_{v}$, as well as for their convex hull $Z(t, x)$, that is, for the set $Z(t, x)$ of all $u=p_{1} u_{1}+\cdots+p_{v} u_{v}$ with $u_{j} \in U_{j}(t, x), p_{j} \geqq 0, j=1, \cdots, v, p_{1}+\cdots+p_{v}=1$.

The proof is straightforward.

(B) The property $(Q)$. Let $U(t, x),(t, x) \in A, U(t, x) \subset E_{m}$, be any variable subset of $E_{m}$, which is a function of $(t, x)$ in $A$. By using the same notations as in $(A)$, we shall say that $U(t, x)$ has property $(Q)$ at $(\bar{t}, \bar{x})$ in $A$, if

$$
U(t, x)=\bigcap_{\delta>0} \operatorname{clco} U(\bar{t}, \bar{x}, \delta) .
$$

We shall say that $U(t, x)$ has property $(Q)$ in $A$ if $U(t, x)$ has property $(Q)$ at every $(t, x)$ of $A$.

(ix) Property $(Q)$ at some $(\bar{t}, \bar{x})$ implies property $(U)$ at the same $(\bar{t}, \bar{x})$, and

$$
U(\bar{t}, \bar{x})=\bigcap_{\delta} \operatorname{clco} U(\bar{t}, \bar{x}, \delta)=\bigcap_{\delta} \operatorname{cl} U(\bar{t}, \bar{x}, \delta)=\bigcap_{\delta} U(\bar{t}, \bar{x}, \delta) .
$$

Analogously, if $U(t, x)$ has property $(U)$ at $(\bar{t}, \bar{x})$ then

$$
U(\bar{t}, \bar{x})=\bigcap_{\delta} \operatorname{cl} U(\bar{t}, \bar{x}, \delta)=\bigcap_{\delta} U(\bar{t}, \bar{x}, \delta) .
$$

Indeed

$$
U(\bar{t}, \bar{x}) \subset \bigcap_{\delta>0} U(\bar{t}, \bar{x}, \delta) \subset \bigcap_{\delta>0} \operatorname{cl} U(\bar{t}, \bar{x}, \delta) \subset \bigcap_{\delta>0} \operatorname{clco} U(\bar{t}, \bar{x}, \delta),
$$

where first and last sets coincide by property $(Q)$ at $(\bar{t}, \bar{x})$, and hence the inclusion 
signs $\subset$ can be replaced by $=$ signs. An analogous argument holds for the second part of the statement.

(x) If $A$ is closed, and $U(t, x)$ is any variable set which is a function of $(t, x)$ in $A$ and has property $(Q)$ in $A$, then the set $M$ of all $(t, x, u) \in A \times E_{m}$ with $u \in U(t, x),(t, x) \in A$, is closed.

Under the hypothesis of (i) the set $M$ is closed but not necessarily compact as the trivial example $U(t, x)=E_{m}, M=A \times E_{m}$ shows. Nevertheless, the statement holds:

(xi) If $A$ is compact, if the set $U(t, x)$ is compact for every $(t, x) \in A$ and possesses property $(Q)$ in $A$, then the set $M$ is compact.

Proof. If $M$ is not compact, then there is some sequence, $\left(t_{k}, x_{k}, u_{k}\right) \in M$, $k=1,2, \cdots$, with $\left(t_{k}, x_{k}\right) \in A,\left|t_{k}\right|+\left|x_{k}\right|+\left|u_{k}\right| \rightarrow+\infty$ as $k \rightarrow \infty$. Since $A$ is compact and hence bounded, we have $\left|u_{k}\right| \rightarrow+\infty$. On the other hand, there is some subsequence, say still $\left(t_{k}, x_{k}\right)$, with $t_{k} \rightarrow \bar{t}, \bar{x}_{k} \rightarrow \bar{x},(\bar{t}, \bar{x}) \in A$. Given $\varepsilon>0$, we have then $u_{k} \in U(\bar{t}, \bar{x}, \varepsilon)$ for all $k$ sufficiently large. Since $U(\bar{t}, \bar{x})$ is compact, there is a solid sphere $S$ containing all of $U(\bar{t}, \bar{x})$ in its interior, say $U(\bar{t}, \bar{x}) \subset$ int $S \subset E_{m}$. On the other hand, if $u \in U(\bar{t}, \bar{x})$, we have $u \in \operatorname{int} S$, and $u_{k} \in E_{m}-S$ again for $k$ large. Since both $u$ and $u_{k}$ belong to the convex set clco $U(t, x, \varepsilon)$, we have $u_{k}^{\prime} \in \operatorname{clco} U(t, x, \varepsilon)$ where $u_{k}^{\prime}$ is the point of intersection of the segment $u u_{k}$ with the boundary bd $S$ of $S$. If $u^{\prime}$ is any point of accumulation of $\left[u_{k}^{i}\right]$, then $u^{\prime} \in b d S$, and $u^{\prime} \in \operatorname{clco} U(\bar{t}, \bar{x}, \varepsilon)$ for every $\varepsilon>0$. Hence $u^{\prime} \varepsilon \bigcap_{\varepsilon} \operatorname{clco} U(\bar{t}, \bar{x}, \varepsilon)$ $=U(\bar{t}, \bar{x})$, a contradiction, since $U(\bar{t}, \bar{x}) \subset$ int $S$. We have proved that $M$ is compact.

(xii) If for every $(t, x) \in A$ the set $U(t, x)$ is closed and convex, and $U(t, x)$ is an upper semicontinuous function of $(t, x)$ in $A$, then $U(t, x)$ has property $(Q)$ in $A$.

Proof. By hypothesis $U(t, x, \delta) \subset[U(t, x)]_{\varepsilon}$, where $U_{\varepsilon}$ is closed and convex as the closed $\varepsilon$-neighborhood of a closed convex set. Hence, $\bigcap_{\delta} \operatorname{clco} U(t, x, \delta)$ $\subset[U(t, x)]_{\varepsilon}$ for every $\varepsilon>0$. Since $U(t, x)$ is closed, then $[U(t, x)]_{\varepsilon} \rightarrow U(t, x)$ as $\varepsilon \rightarrow 0+$. Thus $\bigcap_{\delta} \operatorname{clco} U(t, x, \delta) \subset U(t, x)$. Since the opposite inclusion relation $\supset$ is trivial, we have $\bigcap_{\delta} \operatorname{clco} U(t, x, \delta)=U(t, x)$.

(C) Relations between properties of $U(t, x)$ and of $Q(t, x)$. Let us now consider sets $Q(t, x)=f(t, x, U(t, x)),(t, x) \in A, Q(t, x) \subset E_{n}$, which are the images of sets $U(t, x) \subset E_{m}$ for every $(t, x) \in A$.

The hypothesis that $A$ is compact, that $f$ is continuous on $M$, that $U(t, x)$ has property $(Q)$ [or $(U)]$ in $A$, and that $Q(t, x)$ is convex for every $(t, x) \in A$, does not imply that $Q(t, x)$ has property $(Q)$ [or $(U)]$ in $A$. This can be proved by a simple example. Let $m=n=1, A=[-1 \leqq t \leqq 1,0 \leqq x \leqq 1]$, let $U(t, x)$ be the fixed interval $U=\left[u \in E_{1} \mid 0 \leqq u<+\infty\right]$, and $f=(u+1)^{-1}-t$. Then

$$
Q(t, x)=\left[z \in E_{1} \mid-t<z \leqq 1-t\right],
$$

and, if $-1+\delta<t<1-\delta$,

$$
\operatorname{clco} Q(t, x, \delta)=[-t-\delta \leqq z \leqq 1-t+\delta] .
$$


The intersection of all these sets for $\delta>0$ is the closed set

$$
\left[z \in E_{1} \mid-t \leqq z \leqq 1-t\right]
$$

which is larger than $Q(t, x)$, and thus $Q$ has not property $(Q)$ in $A$. Actually, $Q(t, x)$ is not closed, and hence $Q(t, x)$ has neither property $(Q)$, nor property $(U)$.

Even the stronger hypothesis that $A$ is compact, that $f$ is continuous on $M$, that $U(t, x)$ has property $(Q)$ in $A$, and that $Q(t, x)$ is compact and convex for every $(t, x) \in A$, does not imply that $Q(t, x)$ has property $(Q)$ in $A$. This can be proved by the following example. Let $m=1, n=1, A=\left[(t, x) \in E_{2}, 0 \leqq t \leqq 1\right.$, $0 \leqq x \leqq 1], \quad U=U(t, x)=\left[u \in E_{1} \mid 0 \leqq u<+\infty\right]$, and $f(t, x, u)=[\sin t u]^{2}$, $(t, x, u) \in A \times U$. For $t=0$ we have $f \equiv 0$, hence $Q(0, x)=[z=0]$. For $0<t \leqq 1$, we have $Q(t, x)=[0 \leqq z \leqq 1]$. All sets $Q(t, x)$ are compact and convex, but $Q(t, x)$ does not satisfy property $(Q)$ nor property $(U)$ in $A$.

(xiii) If $A$ is closed and $f$ continuous on $M$, if $U(t, x)$ is compact for every $(t, x) \in A$ and $U(t, x)$ is an upper semicontinuous function of $(t, x)$, then $Q(t, x)$ possesses the same property, and also has property $(U)$. If we know that $Q(t, x)$ is convex, then $Q(t, x)$ has also property $(Q)$.

Proof. Each set $Q(t, x)$ is a compact subset of $E_{n}$ as the continuous image of the compact set $U(t, x)$. The set $U(t, x)$ satisfies property $(U)$ because of (vi), and hence $M$ is closed because of (ii).

Let us prove that $Q(t, x)$ is an upper semicontinuous function of $(t, x)$. Given $(t, x) \in A$ and $\varepsilon>0$, let $\delta=\delta(t, x, \varepsilon)>0$ be the number relative to the definition of upper semicontinuity of $U(t, x)$, and let $M^{\prime}$ be the set of all $\left(t^{\prime}, x^{\prime}, u^{\prime}\right)$ with $\left(t^{\prime}, x^{\prime}\right) \in N_{\delta}(t, x), \quad u^{\prime} \in U\left(t^{\prime}, x^{\prime}\right)$, and $M^{\prime \prime}$ be the set of all $\left(t^{\prime}, x^{\prime}, u^{\prime}\right)$ with $\left(t^{\prime}, x^{\prime}\right) \in N_{\delta}(t, x), u^{\prime} \in[U(t, x)]_{\varepsilon}$. Since $U(t, x)$ is compact, also $[U(t, x)]_{\varepsilon}$ is compact. Let $M^{\prime \prime}=N_{\delta}(t, x) \times[U(t, x)]_{\varepsilon}$, and we have $M^{\prime}=M \cap M^{\prime \prime}$. The set $M^{\prime}$ is compact as the intersection of the closed set $M$ with the compact cylinder $M^{\prime \prime}$. The function $f$ is continuous on $M^{\prime}$ and hence bounded and uniformly continuous. Hence, there is some $\eta, 0<\eta \leqq \min [\delta, \varepsilon]$, such that $\left(t^{\prime \prime}, x^{\prime \prime}\right) \in N_{\eta}\left(t^{\prime}, x^{\prime}\right), \quad\left|u^{\prime}-u^{\prime \prime}\right| \leqq \eta$, $\left(t^{\prime}, x^{\prime}, u^{\prime}\right), \quad\left(t^{\prime \prime}, x^{\prime \prime}, u^{\prime \prime}\right) \in M^{\prime}$ implies $\left|f\left(t^{\prime}, x^{\prime}, u^{\prime}\right)-f\left(t^{\prime \prime}, x^{\prime \prime}, u^{\prime \prime}\right)\right| \leqq \varepsilon$. Also, let $\sigma$ $=\min [\eta, \delta(t, x, \eta)]$. Then, for every $\left(t^{\prime}, x^{\prime}\right) \in N_{\sigma}(t, x)$, we have $U\left(t^{\prime}, x^{\prime}\right) \in[U(t, x)]_{\eta}$, hence, if $u^{\prime} \in U\left(t^{\prime}, x^{\prime}\right)$, there is some $u^{\prime \prime} \in U(t, x)$ with $\left|u^{\prime}-u^{\prime \prime}\right| \leqq \eta$, and finally $\left|f\left(t^{\prime}, x^{\prime}, u^{\prime}\right)-f\left(t, x, u^{\prime \prime}\right)\right| \leqq \varepsilon$. Thus, $Q\left(t^{\prime}, x^{\prime}\right) \subset[Q(t, x)]_{\varepsilon}$ for every $\left(t^{\prime}, x^{\prime}\right) \in N_{\sigma}(t, x)$. This proves that $Q(t, x)$ is an upper semicontinuous function of $(t, x)$. The last part of statement (xiii) is now a consequence of statements (vi) and (xii).

REMARK. The statements and examples above show that properties $(U)$ and $(Q)$ are generalizations of the concept of upper semicontinuity for closed, or closed and convex sets, respectively.

(xiv) If $A$ is a closed subset of the $t x$-space $E_{1} \times E_{n}$, if $U(t, x),(t, x) \in A, U(t, x)$ $\subset E_{m}$, is a variable subset of $E_{m}$ satisfying property $(U)$ in $A$, if $M$ denotes the set of all $(t, x, u)$ with $(t, x) \in A, u \in U(t, x)$, if $f_{0}$ is a continuous scalar function 
from $M$ into the reals, if $\tilde{U}(t, x)$ denotes the variable subset of $E_{m+1}$ defined by $\tilde{U}(t, x)=\left[\tilde{u}=\left(u^{0}, u\right) \in E_{m+1} \mid u^{0} \geqq f_{0}(t, x, u), u \in U(t, x)\right]$, then $\tilde{U}(t, x)$ satisfies property $(U)$.

Proof. First, let us prove that each set $\tilde{U}\left(t_{0}, x_{0}, \delta\right)$ is closed. Indeed, if $\tilde{u}=\left(u^{0}, u\right)$ is a point of accumulation of $\tilde{U}\left(t_{0}, x_{0}, \delta\right)$, then there is a sequence $\tilde{u}_{k}=\left(u_{k}^{0}, u_{k}\right)$ with $u_{k}^{0} \rightarrow u^{0}, u_{k} \rightarrow u, \tilde{u}_{k} \in \tilde{U}\left(t_{0}, x_{0}, \delta\right)$. Hence, there is a corresponding sequence of points $\left(t_{k}, x_{k}\right) \in N_{\delta}\left(t_{0}, x_{0}\right)$ with $u_{k}^{0} \geqq f_{0}\left(t_{k}, x_{k}, u_{k}\right), u_{k} \in U\left(t_{k}, x_{k}\right)$. Thus $u_{k} \in U\left(t_{0}, x_{0}, \delta\right)$. Since $N_{\delta}\left(t_{0}, x_{0}\right)$ is a compact part of the closed set $A$, there is a subsequence, say still $\left(t_{k}, x_{k}\right)$, with $t_{k} \rightarrow \bar{t}, x_{k} \rightarrow \bar{x},(\bar{t}, \bar{x}) \in N_{\delta}\left(t_{0}, x_{0}\right) \subset A$. Thus $\left(t_{k}, x_{k}, u_{k}\right) \in M,\left(t_{k}, x_{k}, u_{k}\right) \rightarrow(\bar{t}, \bar{x}, u)$, and $M$ is a closed set by force of (ii). By the continuity of $f_{0}$ we have then $(\bar{t}, \bar{x}, u) \in M, u \in U(\bar{t}, \bar{x}), u^{0} \geqq f_{0}(\bar{t}, \bar{x}, u)$. Thus $\tilde{u}=\left(u^{0}, u\right) \in \tilde{U}(\tilde{t}, \bar{x})$, and $\tilde{u} \in \tilde{U}\left(t_{0}, x_{0}, \delta\right)$.

Now let $\tilde{u}=\left(u^{0}, u\right)$ be a point $\tilde{u} \in \bigcap_{\delta} \operatorname{cl} \tilde{U}\left(t_{0}, x_{0}, \delta\right)$. Thus, there is a sequence of numbers $\delta_{k}>0, \delta_{k} \rightarrow 0$, with $\tilde{u} \in \operatorname{cl} \tilde{U}\left(t_{0}, x_{0}, \delta_{k}\right)$, and hence $\tilde{u} \in \tilde{U}\left(t_{0}, x_{0}, \delta_{k}\right)$ because these last sets are closed. Thus, there is also a sequence of points $\left(t_{k}, x_{k}\right) \in N_{\delta_{k}}\left(t_{0}, x_{0}\right)$ with $\tilde{u} \in \tilde{U}\left(t_{k}, x_{k}\right)$, or $u^{0} \geqq f_{0}\left(t_{k}, x_{k}, u\right), u \in U\left(t_{k}, x_{k}\right)$. Hence, for every $\eta>0$, we have $u \in U\left(t_{0}, x_{0}, \eta\right)$ for every $k$ sufficiently large (so that $\left.\delta_{k} \leqq \eta\right)$, and, by property $(U)$ of $U(t, x)$ at $\left(t_{0}, x_{0}\right)$, also $u \in \bigcap_{\eta} \operatorname{cl} U\left(t_{0}, x_{0}, \eta\right)$ $=U\left(t_{0}, x_{0}\right)$. Thus, $u \in U\left(t_{0}, x_{0}\right),\left(t_{0}, x_{0}, u\right) \in M$, and by $u^{0} \geqq f_{0}\left(t_{k}, x_{k}, u\right)$ and the continuity of $f_{0}$, also $u^{0} \geqq f_{0}\left(t_{0}, x_{0}, u\right)$. We have proved that $\tilde{u}=\left(u^{0}, u\right) \in \tilde{U}\left(t_{0}, x_{0}\right)$, hence

$$
\bigcap_{\delta} \operatorname{cl} \tilde{U}\left(t_{0}, x_{0}, \delta\right) \subset \tilde{U}\left(t_{0}, x_{0}\right) .
$$

Since the opposite inclusion relation is trivial, equality sign holds, and $\tilde{U}(t, x)$ has property $(U)$ at $\left(t_{0}, x_{0}\right)$, and, thus, everywhere in $A$. Statement (xiv) is thereby proved.

The set $\tilde{U}(t, x)$ of statement (xiv) has not necessarily property $(Q)$ even if we assume that $U(t, x)$ has property $(Q)$ and $f_{0}(t, x, u)$ is convex in $u$ for every $(t, x) \in A$. This can be seen by a simple example. Let $A=[-1 \leqq t \leqq 1,0 \leqq x \leqq 1]$ and let $U=U(t, x)$ be the fixed set $U(t, x)=E_{1}$, that is, $U=\left[-\infty<u^{1}<+\infty\right]$. Then, each set $U(t, x)$ is closed and convex, and obviously $U(t, x)$ possesses property $(Q)$, and $M$ is the cylinder of all $(t, x, u)$ wi. $(t, x) \in A, u \in E_{1}$. Finally, let $f_{0}(t, x, u)=t u^{1}$, so that $f_{0}$ is continuous in $M$ and, for every $(t, x) \in A, f_{0}=t u^{1}$ is linear in $u^{1}$, hence certainly convex in $u^{1}$. Now we have, for $\delta \leqq 1$,

$$
\begin{gathered}
\tilde{U}(t, x)=\left[\left(u^{0}, u^{1}\right) \in E_{2} \mid-\infty<u^{1}<+\infty, t u^{1} \leqq u^{0}<+\infty\right], \\
\tilde{U}(0, x, \delta)=\left[\left(u^{0}, u^{1}\right) \in E_{2}\left|-\infty<u^{1}<+\infty,-\delta\right| u^{1} \mid \leqq u^{0}<+\infty\right] .
\end{gathered}
$$

Consequently, co $\tilde{U}(0, x, \delta)=E_{2}$, and hence

while

$$
\bigcap_{\delta} \operatorname{clco} \tilde{U}(0, x, \delta)=E_{2},
$$




$$
\tilde{U}(0, x)=\left[\left(u^{0}, u^{1}\right) \in E_{2} \mid-\infty<u^{1}<+\infty, u^{0} \geqq 0\right] .
$$

This shows that $\tilde{U}(t, x)$ does not have property $(Q)$ at the points $(0, x)$ of $A$.

A scalar function $f_{0}(t, x, u),(t, x, u) \in M$, is said to be convex in $u$ at $\left(t_{0}, x_{0}\right) \in A$ if

whenever

$$
f_{0}\left(t_{0}, x_{0}, u_{0}\right) \leqq \sum_{i=1}^{N} \lambda_{i} f_{0}\left(t_{0}, x_{0}, u_{i}\right)
$$

$$
u_{0}=\sum_{i=1}^{N} \lambda_{i} u_{i}
$$

where $u_{i} \in U\left(t_{0}, x_{0}\right), \lambda_{i} \geqq 0, i=1, \cdots, N, \lambda_{1}+\cdots+\lambda_{N}=1$.

A scalar function $f_{0}(t, x, u),(t, x, u) \in M$, is said to be quasi-normally convex in $u$ at $\left(t_{0}, x_{0}, u_{0}\right) \in M$ provided, given $\varepsilon>0$, there are a number $\delta=\delta\left(t_{0}, x_{0}, u_{0}, \varepsilon\right)$ $>0$, and a linear scalar function $z(u)=r+b \cdot u, b=\left(b_{1}, \cdots, b_{m}\right), r, b_{1}, \cdots, b_{m}$ real, such that

(a) $f_{0}(t, x, u) \geqq z(u)$ for all $(t, x) \in N_{\delta}\left(t_{0}, x_{0}\right), u \in U(t, x)$,

(b) $f_{0}(t, x, u) \leqq z(u)+\varepsilon$ for all $(t, x) \in N_{\delta}\left(t_{0}, x_{0}\right), u \in U(t, x),\left|u-u_{0}\right| \leqq \delta$.

The scalar function $f_{0}(t, x, u)$ is said to be normally convex in $u$ at $\left(t_{0}, x_{0}, u_{0}\right)$ if, given $\varepsilon>0$, there are numbers $\delta=\delta\left(t_{0}, x_{0}, u_{0}, \varepsilon\right)>0, v=v\left(t_{0}, x_{0}, u_{0}, \varepsilon\right)>0$, and a linear scalar function $z(u)=r+b \cdot u$ as above such that (b) holds and

(a') $f_{0}(t, x, u) \geqq z(u)+v\left|u-u_{0}\right|$ for all $(t, x) \in N_{\delta}\left(t_{0}, x_{0}\right), u \in U(t, x)$.

The scalar function $f_{0}(t, x, u)$ is said to be quasi-normally convex in $u$, or normally convex in $u$, if it has these properties at every $\left(t_{0}, x_{0}, u_{0}\right) \in M$.

For the case where $U=U(t, x)$ is the fixed set $U=E_{m}$, the following statement gives a useful characterization of the functions $f_{0}$ which are normally convex in $u$.

(xv) If $A$ is closed, and $f_{0}(t, x, u)$ is continuous on $M=A \times E_{m}$, then $f_{0}$ is normally convex in $u$ if and only if $f_{0}$ is convex in $u$ at every $\left(t_{0}, x_{0}\right) \in A$, and for no points $\left(t_{0}, x_{0}\right) \in A, u_{0}, u_{1} \in E_{m}, u_{1} \neq 0$, the relation holds $f_{0}\left(t_{0}, x_{0}, u_{0}\right)$ $=2^{-1}\left[f_{0}\left(t_{0}, x_{0}, u_{0}+\lambda u_{1}\right)+f_{0}\left(t_{0}, x_{0}, u_{0}-\lambda u_{1}\right)\right]$ for all $\lambda \geqq 0$.

This statement was proved in [9a] and [10]. In particular, if for every $(t, x) \in A, f_{0}(t, x, u)$ is convex in $u$ and $f_{0}(t, x, u)|| u \mid \rightarrow+\infty$, as $|u| \rightarrow+\infty$, then certainly $f_{0}(t, x, u)$ is normally convex in $u$.

(xvi) If $A$ is a closed subset of the $t x$-space $E_{1} \times E_{n}$, if $U(t, x),(t, x) \in A$, $U(t, x) \subset E_{m}$, is a variable subset of $E_{m}$ satisfying property $(Q)$ in $A$, if $M$ denotes the set of all $(t, x, u)$ with $(t, x) \in A, u \in U(t, x)$, if $f_{0}$ is a continuous function from $M$ into the reals, which is convex in $u$ for every $(t, x) \in A$, if either $(\alpha)$ the sets $U(t, x)$ are all contained in a fixed solid sphere $S$ of $E_{m}$, or $(\beta)$ the function $f_{0}(t, x, u)$ is quasi-normally convex in $u$ at every $\left(t_{0}, x_{0}, u_{0}\right)$ of $M$, then the set $\tilde{\sigma}(t, x)$ of statement (xiv) has property $(Q)$ in $A$.

Proof. Let $\tilde{u}=\left(u^{0}, u\right)$ be a point $\tilde{u}=\bigcap_{\delta} \operatorname{clco} \tilde{U}\left(t_{0}, x_{0}, \delta\right)$. Then there is a sequence $\left[\delta_{k}\right]$ of numbers $\delta_{k}>0, \delta_{k} \rightarrow 0$, with $\tilde{u} \in \operatorname{clco} \tilde{U}\left(t_{0}, x_{0}, \delta_{k}\right)$. Hence, 
there is a sequence of pairs of points $\tilde{u}_{k 1}, \tilde{u}_{k 2} \in E_{m+1}$ and of points $\tilde{v}_{k}$ of the segment $\left(\tilde{u}_{k 1} \tilde{u}_{k 2}\right) \in E_{m+1}$, such that

$$
\begin{gathered}
\tilde{v}_{k} \rightarrow \tilde{u}, \quad \tilde{u}_{k 1}, \tilde{u}_{k 2} \in \tilde{U}\left(t_{0}, x_{0}, \delta_{k}\right), \\
\tilde{v}_{k}=\alpha_{k} \tilde{u}_{k 1}+\left(1-\alpha_{k}\right) \tilde{u}_{k 2}, \quad 0 \leqq \alpha_{k} \leqq 1, \quad k=1,2, \cdots .
\end{gathered}
$$

We shall use the notation $\tilde{v}_{k}=\left(v_{k}^{0}, v_{k}\right), \tilde{u}=\left(u^{0}, u\right), \tilde{u}_{k j}=\left(u_{k j}^{0}, u_{k j}\right), j=1,2$. Then we have

$$
\begin{array}{cl}
v_{k}^{0} \rightarrow u^{0}, v_{k} \rightarrow u, & u_{k 1}, u_{k 2} \in U\left(t_{0}, x_{0}, \delta_{k}\right), \\
v_{k}^{0}=\alpha_{k} u_{k 1}^{0}+\left(1-\alpha_{k}\right) u_{k 2}^{0}, & v_{k}=\alpha_{k} u_{k 1}+\left(1-\alpha_{k}\right) u_{k 2} .
\end{array}
$$

Consequently, there are points such that

$$
\begin{gathered}
\left(t_{k 1}, x_{k 1}\right),\left(t_{k 2}, x_{k 2}\right) \in N_{\delta_{k}}\left(t_{0}, x_{0}\right) \subset A, \\
u_{k 1} \in U\left(t_{k 1}, x_{k 1}\right), \quad u_{k 2} \in U\left(t_{k 2}, x_{k 2}\right) .
\end{gathered}
$$

The sequence $\left[\alpha_{k}\right]$ is bounded, hence there is a convergent subsequence, say still $\alpha_{k}$, so that $\alpha_{k} \rightarrow \alpha$ for some $0 \leqq \alpha \leqq 1$.

For every $\eta>0$ and $k$ sufficiently large (so that $\delta_{k} \leqq \eta$ ), we have $u_{k 1}, u_{k 2} \in U\left(t_{0}, x_{0}, \eta\right)$, hence

$$
u_{k 1}, u_{k 2} \in \operatorname{cl} \operatorname{co} U\left(t_{0}, x_{0}, \eta\right)
$$

As a consequence

$$
v_{k}=\alpha_{k} u_{k 1}+\left(1-\alpha_{k}\right) u_{k 2} \in \operatorname{clco} U\left(t_{0}, x_{0}, \eta\right)
$$

for all $k$ sufficiently large. As $k \rightarrow \infty$, we obtain $u \in \operatorname{clco} U\left(t_{0}, x_{0}, \eta\right)$. By the property $(Q)$, finally

$$
u \in \bigcap_{\eta} \operatorname{clco} U\left(t_{0}, x_{0}, \eta\right)=U\left(t_{0}, x_{0}\right) \text {. }
$$

Assume first that condition $(\alpha)$ holds. Then both sequences $\left[u_{k 1}\right],\left[u_{k 2}\right]$ are bounded, and hence there is a subsequence, say still $\left[u_{k 1}\right],\left[u_{k 2}\right]$, for which both $u_{k 1}$ and $u_{k 2}$ are convergent in $E_{m}$, say $u_{k 1} \rightarrow u_{1}, u_{k 2} \rightarrow u_{2}, u_{1}, u_{2} \in E_{m}$. For such a subsequence, we have

$$
\begin{gathered}
v_{k}^{0}=\alpha_{k} u_{k 1}^{0}+\left(1-\alpha_{k}\right) u_{k 2}^{0} \geqq \alpha_{k} f_{0}\left(t_{k 1}, x_{k 1}, u_{k 1}\right)+\left(1-\alpha_{k}\right) f_{0}\left(t_{k 2}, x_{k 2}, u_{k 2}\right), \\
v_{k}=\alpha_{k} u_{k 1}+\left(1-\alpha_{k}\right) u_{k 2}, \\
\left(t_{k 1}, x_{k 1}, u_{k 1}\right),\left(t_{k 2}, x_{k 2}, u_{k 2}\right) \in M,
\end{gathered}
$$

where $M$ is closed. By taking limits as $k \rightarrow \infty$, we have

$$
\begin{gathered}
u^{0} \geqq \alpha f_{0}\left(t_{0}, x_{0}, u_{1}\right)+(1-\alpha) f_{0}\left(t_{0}, x_{0}, u_{2}\right), \\
u=\alpha u_{1}+(1-\alpha) u_{2}, \\
\left(t_{0}, x_{0}, u_{1}\right),\left(t_{0}, x_{0}, u_{2}\right) \in M .
\end{gathered}
$$


By the convexity of $f_{0}$ in $u$ at $\left(t_{0}, x_{0}\right)$ we have now

$$
u^{0} \geqq f_{0}\left(t_{0}, x_{0}, \alpha u_{1}+(1-\alpha) u_{2}\right)=f_{0}\left(t_{0}, x_{0}, u\right) .
$$

This proves that $\tilde{u}=\left(u^{0}, u\right) \in \tilde{U}\left(t_{0}, x_{0}\right)$, hence

$$
\bigcap_{\delta} \operatorname{clco} \tilde{U}\left(t_{0}, x_{0}, \delta\right) \subset \tilde{U}\left(t_{0}, x_{0}\right) \text {. }
$$

Since the opposite inclusion is trivial, $=$ sign holds in this relation, and $\tilde{U}(t, x)$ has property $(Q)$ at $\left(t_{0}, x_{0}\right)$. Since $\left(t_{0}, x_{0}\right) \in A$ is arbitrary, $\tilde{U}(t, x)$ has property (Q) in $A$.

Assume now that condition $(\beta)$ holds. As stated by relation (1) above, $u \in U\left(t_{0}, x_{0}\right)$, hence $\left(t_{0}, x_{0}, u\right) \in M$. By the quasi-normal convexity of $f_{0}$ in $u$ at $\left(t_{0}, x_{0}, u\right)$ we deduce the existence of a number $\delta>0$ and of a linear scalar function $z(v)=r+b \cdot v$ such that (a) $f_{0}(t, x, v) \geqq z(v)$ for all $(t, x) \in N_{\delta}\left(t_{0}, x_{0}\right), v \in U(t, x)$ and (b) $f_{0}(t, x, v) \leqq z(v)+\varepsilon$ for all $(t, x) \in N_{\delta}\left(t_{0}, x_{0}\right), v \in U(t, x),|u-v| \leqq \delta$. By combining (a) and (b) we have then (c) $z(u) \leqq f_{0}\left(t_{0}, x_{0}, u\right) \leqq z(u)+\varepsilon$.

Now we have $v_{k}=\alpha_{k} u_{k 1}+\left(1-\alpha_{k}\right) u_{k 2}$ for some $0 \leqq \alpha_{k} \leqq 1$, and $v_{k} \rightarrow u$, $\left(t_{k j}, x_{k j}\right) \rightarrow\left(t_{0}, x_{0}\right), j=1,2$. Thus, for $k$ sufficiently large, $\left(t_{k j}, x_{k j}\right) \in N_{\delta}\left(t_{0}, x_{0}\right)$, $j=1,2$, and, by property (a),

$$
\begin{aligned}
v_{k}^{0} & \geqq \alpha_{k} f_{0}\left(t_{k 1}, x_{k 1}, u_{k 1}\right)+\left(1-\alpha_{k}\right) f_{0}\left(t_{k 2}, x_{k 2}, u_{k 2}\right) \\
& \geqq \alpha_{k} z\left(u_{k 1}\right)+\left(1-\alpha_{k}\right) z\left(u_{k 2}\right) \\
& \geqq z\left(\alpha_{k} u_{k 1}+\left(1-\alpha_{k}\right) u_{k 2}\right)=z\left(v_{k}\right) .
\end{aligned}
$$

As $k \rightarrow+\infty$, we have then $u^{0} \geqq z(u)$, and finally by (b) above, $u^{0} \geqq f_{0}\left(t_{0}, x_{0}, u\right)$ $-\varepsilon$, where $\varepsilon>0$ is arbitrary. We conclude that $u^{0} \geqq f_{0}\left(t_{0}, x_{0}, u\right)$, with $u \in U\left(t_{0}, x_{0}\right)$. Thus $\tilde{u}=\left(u^{0}, u\right) \in \tilde{U}\left(t_{0}, x_{0}\right)$, and again we have proved inclusion (2). The same reasoning above yields that $\tilde{U}(t, x)$ has property $(Q)$ in $A$.

(xvii) If $A$ is a closed subset of the $t x$-space $E_{1} \times E_{n}$, if $U(t, x),(t, x) \in A$, is a variable subset of $E_{m}$ satisfying property $(U)$ in $A$, if $M$ denotes the set of all $(t, x, u)$ with $(t, x) \in A, u \in U(t, x)$, if $\tilde{f}=\left(f_{0}, f\right)$ is a continuous function from $M$ into the $\tilde{z}$-space $E_{n+1}, \tilde{z}=\left(z^{0}, z\right)$, if $Q(t, x) \subset E_{n}, \tilde{\tilde{Q}}(t, x) \subset E_{n+1}$ are the sets

$$
\begin{aligned}
& Q(t, x)=f(t, x, U(t, x))=\left[z \in E_{n} \mid z=f(t, x, u), u \in U(t, x)\right], \\
& \tilde{\tilde{Q}}(t, x)=\left[\tilde{z}=\left(z^{0}, z\right) \in E_{n+1} \mid z^{0} \geqq f_{0}(t, x, u), z=f(t, x, u), u \in U(t, x)\right],
\end{aligned}
$$

and (a) for every $(t, x) \in A, Q(t, x)$ is a convex subset of $E_{n}$; (b) $Q(t, x)$ has property $(Q)$ in $A$; (c) for every $(t, x) \in A, z=f(t, x, u)$ is a 1-1 map from $U(t, x)$ onto $Q(t, x)$ with a continuous inverse $u=f^{-1}(t, x, z), z \in Q(t, x)$; (d) the real valued function $F_{0}(t, x, z)=f_{0}\left(t, x, f^{-1}(t, x, z)\right),(t, x) \in A, z \in Q(t, x)$, is continu-

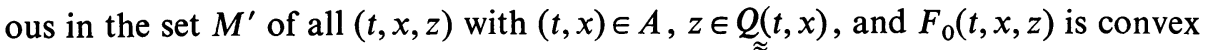
in $z$ and also quasi-normally convex, then the set $\tilde{Q}(t, x)$ is convex and has property $(Q)$ in $A$. 
Proof. Indeed, under the specific hypotheses above, the set $\tilde{\tilde{Q}}(t, x)$ can be represented as

$$
\tilde{Q}(t, x)=\left[\tilde{z}=\left(z^{0}, z\right) \in E_{n+1} \mid z^{0} \geqq F_{0}(t, x, z), z \in Q(t, x)\right],
$$

and thus $\tilde{\tilde{Q}}$ is generated from $Q(t, x)$ exactly as $\tilde{U}$ is generated from $U(t, x)$. By statement (xvi) above we conclude that $\tilde{\tilde{Q}}(t, x)$ has property $(Q)$ in $A$.

REMARK. The condition that $f$ is a homeomorphism between $U$ and $Q$ is certainly verified in all free problems, where $m=n, f=u$, that is, $f_{i}=u_{i}$, $i=1,2, \cdots, n$ (see $\$ 11$ below). In this situation then we have $F_{0}(t, x, u)=f_{0}(t, x, u)$, and the convexity of $f_{0}$ in $u$ implies the convexity of $F_{0}$ in $u$. We shall need this remark, and the more general statement (xvi) in $\$ 11$.

5. Closure theorems. We shall use here the notations of $\S \S 2$ and 3. In particular, a trajectory $x(t)$ is defined as in $\S 3$.

Closure Theorem I. Let $A$ be a closed subset of $E_{1} \times E_{n}$, let $U(t, x)$ be a closed subset of $E_{m}$ for every $(t, x) \in A$, let $f(t, x, u)=\left(f_{1}, \cdots, f_{n}\right)$ be a continuous vector function on $M$ into $E_{n}$, and let $Q(t, x)=f(t, x, U(t, x))$ be a closed convex subset of $E_{n}$ for every $(t, x) \in A$. Assume that $U(t, x)$ has property $(U)$ in $A$, and that $Q(t, x)$ has property $(Q)$ in $A$. Let $x_{k}(t), t_{1 k} \leqq t \leqq t_{2 k}, k=1,2, \cdots$, be a sequence of trajectories, which is convergent in the metric $\rho$ toward an absolutely continuous function $x(t), t_{1} \leqq t \leqq t_{2}$. Then $x(t)$ is a trajectory.

REMARK. If we assume that $U(t, x)$ is compact for every $(t, x) \in A$, and that $U(t, x)$ is an upper semicontinuous function of $(t, x)$ in $A$, then by statement (xiii), the set $Q(t, x)$ has the same property, $U(t, x)$ has property $(U), Q(t, x)$ has property $(Q)$, and Closure Theorem I reduces to one of A. F. Filippov [2] (not explicitly stated in [2] but contained in the proof of his existence theorem for the Pontryagin problem with $U(t, x)$ always compact).

Proof of Closure Theorem I. The vector functions

$$
\begin{gathered}
\phi(t)=x^{\prime}(t), \quad t_{1} \leqq t \leqq t_{2}, \\
\phi_{k}(t)=x_{k}^{\prime}(t)=f\left(t, x_{k}(t), u_{k}(t)\right), \quad t_{1 k} \leqq t \leqq t_{2 k}, \quad k=1,2, \cdots,
\end{gathered}
$$

are defined almost everywhere and are $L$-integrable. We have to prove that $(t, x(t)) \in A$ for every $t_{1} \leqq t \leqq t_{2}$, and that there is a measurable control function $u(t), t_{1} \leqq t \leqq t_{2}$, such that

$$
\phi(t)=x^{\prime}(t)=f(t, x(t), u(t)), \quad u(t) \in U(t, x(t)),
$$

for almost all $t \in\left[t_{1}, t_{2}\right]$.

First, $\rho\left(x_{k}, x\right) \rightarrow 0$ as $k \rightarrow \infty$; hence, $t_{1 k} \rightarrow t_{1}, t_{2 k} \rightarrow t_{2}$. If $t \in\left(t_{1}, t_{2}\right)$, or $t_{1}<t<t_{2}$, then $t_{1 k}<t<t_{2 k}$ for all $k$ sufficiently large and $\left(t, x_{k}(t)\right) \in A$. Since $x_{k}(t) \rightarrow x(t)$ as $k \rightarrow \infty$ and $A$ is closed, we conclude that $(t, x(t)) \in A$ for every $t_{1}<t<t_{2}$. 
Since $x(t)$ is continuous, and hence continuous at $t_{1}$ and $t_{2}$, we conclude that $(t, x(t)) \in A$ for every $t_{1} \leqq t \leqq t_{2}$.

For almost all $t \in\left[t_{1}, t_{2}\right]$ the derivative $x^{\prime}(t)$ exists and is finite. Let $t_{0}$ be such a point with $t_{1}<t_{0}<t_{2}$. Then there is a $\sigma>0$ with $t_{1}<t_{0}-\sigma<t_{0}+\sigma<t_{2}$, and, for some $k_{0}$ and all $k \geqq k_{0}$, also $t_{1 k}<t_{0}-\sigma<t_{0}+\sigma<t_{2 k}$. Let $x_{0}=x\left(t_{0}\right)$.

We have $x_{k}(t) \rightarrow x(t)$ uniformly in $\left[t_{0}-\sigma, t_{0}+\sigma\right]$ and all functions $x(t), x_{k}(t)$ are continuous in the same interval. Thus, they are equicontinuous in $\left[t_{0}-\sigma, t_{0}+\sigma\right]$. Given $\varepsilon>0$, there is a $\delta>0$ such that $t, t^{\prime} \in\left[t_{0}-\sigma, t_{0}+\sigma\right]$, $\left|t-t^{\prime}\right| \leqq \delta, k \geqq k_{0}$, implies

$$
\left|x(t)-x\left(t^{\prime}\right)\right| \leqq \varepsilon / 2, \quad\left|x_{k}(t)-x_{k}\left(t^{\prime}\right)\right| \leqq \varepsilon / 2 .
$$

We can assume $0<\delta<\sigma, \delta \leqq \varepsilon$. For any $h, 0<h \leqq \delta$, let us consider the averages

$$
m_{h}=h^{-1} \int_{0}^{h} \phi\left(t_{0}+s\right) d s=h^{-1}\left[x\left(t_{0}+h\right)-x\left(t_{0}\right)\right]
$$

$$
m_{h k}=h^{-1} \int_{0}^{h} \phi_{k}\left(t_{0}+s\right) d s=h^{-1}\left[x_{k}\left(t_{0}+h\right)-x_{k}\left(t_{0}\right)\right] .
$$

Given $\eta>0$ arbitrary, we can fix $h, 0<h \leqq \delta<\sigma$, so small that

$$
\left|m_{h}-\phi\left(t_{0}\right)\right| \leqq \eta \text {. }
$$

Having so fixed $h$, let us take $k_{1} \geqq k_{0}$ so large that

$$
\left|m_{h k}-m_{h}\right| \leqq \eta,\left|x_{k}\left(t_{0}\right)-x\left(t_{0}\right)\right| \leqq \varepsilon / 2
$$

for all $k \geqq k_{1}$. This is possible since $x_{k}(t) \rightarrow x(t)$ as $k \rightarrow \infty$ both at $t=t_{0}$ and $t=t_{0}+h$. Finally, for $0 \leqq s \leqq h$,

$$
\begin{aligned}
&\left|x_{k}\left(t_{0}+s\right)-x\left(t_{0}\right)\right| \leqq\left|x_{k}\left(t_{0}+s\right)-x_{k}\left(t_{0}\right)\right|+\left|x_{k}\left(t_{0}\right)-x\left(t_{0}\right)\right| \\
& \leqq \varepsilon / 2+\varepsilon / 2=\varepsilon \\
&\left|\left(t_{0}+s\right)-t_{0}\right| \leqq h \leqq \delta \leqq \varepsilon
\end{aligned}
$$

Hence, by the definition of $Q\left(t_{0}, x_{0}, 2 \varepsilon\right)$, also

$$
\phi_{k}\left(t_{0}+s\right)=f\left(t_{0}+s, x_{k}\left(t_{0}+s\right), u_{k}\left(t_{0}+s\right)\right) \in Q\left(t_{0}, x_{0}, 2 \varepsilon\right) .
$$

The second integral relation (3) shows that we have also

$$
m_{h k} \in \operatorname{cl} \text { co } Q\left(t_{0}, x_{0}, 2 \varepsilon\right),
$$


since the latter is a closed convex set. Finally, by relations (4) and (5), we deduce

$$
\left|\phi\left(t_{0}\right)-m_{h k}\right| \leqq\left|\phi\left(t_{0}\right)-m_{h}\right|+\left|m_{h}-m_{h k}\right| \leqq 2 \eta
$$

and hence

$$
\phi\left(t_{0}\right) \in\left[\operatorname{clco} Q\left(t_{0}, x_{0}, 2 \varepsilon\right)\right]_{2 \eta} .
$$

Here $\eta>0$ is an arbitrary number, and the set in brackets is closed. Hence,

$$
\phi\left(t_{0}\right) \in \operatorname{clco} Q\left(t_{0}, x_{0}, 2 \varepsilon\right),
$$

and this relation holds for every $\varepsilon>0$. By property $(Q)$ we have

$$
\phi\left(t_{0}\right) \in \bigcap_{\varepsilon} \operatorname{clco} Q\left(t_{0}, x_{0}, 2 \varepsilon\right)=Q\left(t_{0}, x_{0}\right),
$$

where $x_{0}=x\left(t_{0}\right)$, and $Q\left(t_{0}, x_{0}\right)=f\left(t_{0}, x_{0}, U\left(t_{0}, x_{0}\right)\right)$. This relation implies that there are points $\bar{u}=\bar{u}\left(t_{0}\right) \in U\left(t_{0}, x_{0}\right)$ such that

$$
\phi\left(t_{0}\right)=f\left(t_{0}, x\left(t_{0}\right), \bar{u}\left(t_{0}\right)\right) .
$$

This holds for almost all $t_{0} \in\left[t_{1}, t_{2}\right]$, that is, for all $t$ of a measurable set $I$ $\subset\left[t_{1}, t_{2}\right]$ with meas $I=t_{2}-t_{1}$. If we take $I_{0}=\left[t_{1}, t_{2}\right]-I$, then meas $I_{0}=0$. Hence, there is at least one function $\bar{u}(t)$, defined almost everywhere in $\left[t_{1}, t_{2}\right]$, for which relation (6) holds a.e. in $\left[t_{1}, t_{2}\right]$. We have to prove that there is at least one such function which is measurable. For every $t \in I$, let $P(t)$ denote the set

$$
P(t)=[u \mid u \in U(t, x(t)), \phi(t)=f(t, x(t), u)] \subset U(t, x(t)) \subset E_{m} .
$$

We have proved that $P(t)$ is not empty.

For every integer $\lambda=1,2, \cdots$, there is a closed subset $C_{\lambda}$ of $I, C_{\lambda} \subset I \subset\left[t_{1}, t_{2}\right]$, with meas $C_{\lambda}>\max \left[0, t_{2}-t_{1}-1 / \lambda\right]$, such that $\phi(t)$ is continuous on $\lambda$. Let $W_{\lambda}$ be the set

$$
W_{\lambda}=\left[(t, u) \mid t \in C_{\lambda}, u \in P(t)\right] \subset E_{1} \times E_{m} .
$$

Let us prove that the set $W_{\lambda}$ is closed. Indeed, if $(\bar{t}, \bar{u})$ is a point of accumulation of $W_{\lambda}$, then there is a sequence $\left(t_{s}, u_{s}\right), s=1,2, \cdots$, with $\left(t_{s}, u_{s}\right) \in W_{\lambda}$, $t_{s} \rightarrow \bar{t}, u_{s} \rightarrow \bar{u}$. Then $t_{s} \in C_{\lambda}$ and $\bar{t} \in C_{\lambda}$ since $C_{\lambda}$ is closed. Also $x\left(t_{s}\right) \rightarrow x(\bar{t})$, $\phi\left(t_{s}\right) \rightarrow \phi(\bar{t})$, and since $\left(t_{s}, x\left(t_{s}\right)\right) \in A, \phi\left(t_{s}\right)=f\left(t_{s}, x\left(t_{s}\right), u_{s}\right), \quad\left(t_{s}, x\left(t_{s}\right), u_{s}\right) \in M$, we have also $(\bar{t}, x(\bar{t})) \in A,(t, x(\bar{t}), \bar{u}) \in M$, because $A$ and $M$ are closed, and $\phi(\bar{t})=f(\bar{t}, x(\bar{t}), \bar{u})$ because $f$ is continuous. Thus, $\bar{u} \in P(\bar{t})$, and $(\bar{t}, x) \in W_{\lambda}$.

For every integer $l$ let $W_{\lambda l}, P_{l}(t)$, be the sets

$$
\begin{aligned}
& W_{\lambda l}=\left[(t, u)\left|(t, u) \in W_{\lambda},\right| u \mid \leqq l\right] \subset W_{\lambda} \subset E_{1} \times E_{m}, \\
& P_{l}(t)=[u|u \in P(t),| u \mid \leqq l] \subset P(t) \subset U(t, x(t)) \subset E_{m}, \\
& C_{\lambda l}=\left[t \mid(t, u) \in W_{\lambda l} \text { for some } u\right] \subset C_{\lambda} \subset I \subset\left[t_{1}, t_{2}\right] .
\end{aligned}
$$


Obviously, $W_{\lambda l}$ is compact, and so is $C_{\lambda l}$ as its projection on the $t$-axis. Also, $\bigcup_{l} C_{\lambda l}=C_{\lambda}$, and $W_{\lambda l}$ is the set of all $(t, u)$ with $t \in C_{\lambda l}, u \in P_{l}(t)$. Thus, for $t \in C_{\lambda l}, P_{l}(t)$ is a compact subset of $U(t, x(t))$.

For $t \in C_{\lambda l}$, the set $P_{l}(t)$ is the nonempty compact subset of all

$$
u=\left(u^{1}, \cdots, u^{m}\right) \in U(t, x(t))
$$

with $f(t, x(t), u)=\phi(t)$, and $|u| \leqq l$. As in Filippov's argument let $P_{1}$ be the subset of $P_{l}(t)$ with $u^{1}$ minimum, let $P_{2}$ be the subset of $P_{1}$ with $u^{2}$ minimum, $\cdots$, let $P_{m}$ be the subset of $P_{m-1}$ with $u^{m}$ minimum. Then $P_{m}$ is a single point $u=u(t) \in U(t, x(t))$ with $u(t)=\left(u^{1}, \cdots, u^{m}\right), t \in C_{\lambda l},|u(t)| \leqq l$, and $f(t, x(t), u(t))$ $=\phi(t)$. Let us prove that $u(t), t \in C_{\lambda l}$, is measurable. We shall prove this by induction on the coordinates. Let us assume that $u^{1}(t), \cdots, u^{s-1}(t)$ have been proved to be measurable on $C_{\lambda l}$ and let us prove that $u^{s}(t)$ is measurable. For $s=1$ nothing is assumed, and the argument below proves that $u^{1}(t)$ is measurable. For every integer $j$ there are closed subsets $C_{\lambda l j}$ of $C_{\lambda l}$ with $C_{\lambda l j} \subset C_{\lambda l}, C_{\lambda l j} \subset C_{\lambda l, j+1}$, meas $C_{\lambda l j} 0>\max \left[0\right.$, meas $\left.C_{\lambda l}-1 / j\right]$, such that $u^{1}(t), \cdots, u^{s-1}(t)$ are continuous on $C_{\lambda l j}$. The function $\phi(t)$ is already continuous on $C_{\lambda}$ and hence $\phi(t)$ is continuous on every set $C_{\lambda l}$ and $C_{\lambda l j}$. Let us prove that $u^{s}(t)$ is measurable on $C_{\lambda l j}$. We have only to prove that, for every real $a$, the set of all $t \in C_{\lambda l j}$ with $u^{s}(t) \leqq a$ is closed. Suppose that this is not the case. Then there is a sequence of points $t_{k} \in C_{\lambda l j}$ with $u^{s}\left(t_{k}\right) \leqq a, t_{k} \rightarrow \bar{t} \in C_{\lambda l j}, u^{s}(\bar{t})>a$. Then $\phi\left(t_{k}\right) \rightarrow \phi(\bar{t}), u^{\alpha}\left(t_{k}\right) \rightarrow u^{\alpha}(\bar{t})$ as $k \rightarrow \infty$, $\alpha=1, \cdots, s-1$. Since $\left|u^{\beta}\left(t_{k}\right)\right| \leqq l$ for all $k$ and $\beta=s, s+1, \cdots, m$, we can select a subsequence, say still $\left[t_{k}\right]$ such that $u^{\beta}\left(t_{k}\right) \rightarrow \tilde{u}^{\beta}$ as $k \rightarrow \infty, \beta=s, s+1, \cdots, m$, for some real numbers $\tilde{u}^{\beta}$. Then $t_{k} \rightarrow \bar{t}, x\left(t_{k}\right) \rightarrow x(\bar{t}), u\left(t_{k}\right) \rightarrow \tilde{u}$, where

$$
\tilde{u}=\left(u^{1}(\bar{t}), \cdots, u^{s-1}(\bar{t}), \tilde{u}^{s}, \cdots, \tilde{u}^{m}\right) .
$$

Then, given any number $\eta>0$, we have

$$
u\left(t_{k}\right) \in U\left(t_{k}, x\left(t_{k}\right)\right) \subset \operatorname{cl} U(\bar{t}, x(\bar{t}), \eta)
$$

for all $k$ sufficiently large, and, as $k \rightarrow \infty$, also

$$
\tilde{u} \in \operatorname{cl} U(\bar{t}, x(\bar{t}), \eta)
$$

By property $(U)$ we have

$$
\tilde{u} \in \bigcap_{\eta} \operatorname{cl} U(\bar{t}, x(\bar{t}), \eta)=U(\bar{t}, x(\bar{t})) .
$$

On the other hand $\phi\left(t_{k}\right)=f\left(t_{k}, x\left(t_{k}\right), u\left(t_{k}\right)\right), u^{s}\left(t_{k}\right) \leqq a$, yield as $k \rightarrow \infty$,

$$
\phi(\bar{t})=f(\bar{t}, x(\bar{t}), \tilde{u}), \quad \tilde{u}^{s} \leqq a,
$$

while $\bar{t} \in C_{\lambda l}$ implies

$$
\phi(\bar{t})=f(\bar{t}, x(\bar{t}), u(\bar{t})), \quad u^{s}(\bar{t})>a .
$$


Relations (7) and (8) are contradictory because of the property of minimum with which $u^{s}(\bar{t})$ has been chosen. Thus $u^{s}(t)$ is measurable on $C_{\lambda l j}$ for every $j$, and then $u^{s}(t)$ is also measurable on $C_{\lambda l}$. By induction argument, all components $u^{1}(t), \cdots, u^{m}(t)$ of $u(t)$ are measurable on $C_{\lambda l}$, hence $u(t)$ is measurable on $C_{\lambda l}$. Since $\bigcup_{l} C_{\lambda l}=C_{\lambda}$, meas $C_{\lambda}>$ meas $I-1 / \lambda$, we conclude that there exists a function $u(t)$ which is measurable on every set $C_{\lambda}$ and hence on $I$, with meas $I=t_{2}-t_{1}$. Thus, $u(t)$ is defined a.e. on $\left[t_{1}, t_{2}\right], u(t) \in U(t, x(t))$, and $f(t, x(t), u(t))=\phi(t)$ a.e. on $\left[t_{1}, t_{2}\right]$. Closure Theorem $I$ is thereby proved.

Let us denote by $y=\left(x^{1}, \cdots, x^{s}\right)$ the $s$-vector made up of certain components, say $x^{1}, \cdots, x^{s}, 0 \leqq s \leqq n$, of $x=\left(x^{1}, \cdots, x^{n}\right)$, and by $z$ the complementary $(n-s)$ vectors $z=\left(x^{s+1}, \cdots, x^{n}\right)$ of $x$, so that $x=(y, z)$. Let us assume that $f(t, y, u)$ depends only on the coordinates $x^{1}, \cdots, x^{s}$ of $x$. If $x(t), t_{1} \leqq t \leqq t_{2}$, is any vector function, we shall denote by $x(t)=[y(t), z(t)]$ the corresponding decomposition of $x(t)$ in its coordinates $y(t)=\left(x^{1}, \cdots, x^{s}\right)$ and $z(t)=\left(x^{s+1}, \cdots, x^{n}\right)$.

We shall denote by $A_{0}$ a closed subset of points $\left(t, x^{1}, \cdots, x^{s}\right)$, that is, a closed subset of the $t y$-space $E_{1} \times E_{s}$, and let $A=A_{0} \times E_{n-s}$. Thus, $A$ is a closed subset of the $t x$-space $E_{1} \times E_{n}$.

Closure Theorem II. Let $A_{0}$ be a closed subset of the ty-space $E_{1} \times E_{s}$, and then $A=A_{0} \times E_{n-s}$ is a closed subset of the tx-space $E_{1} \times E_{n}$. Let $U(t, y)$ denote a closed subset of $E_{m}$ for every $(t, y) \in A_{0}$, let $M_{0}$ be the set of all $(t, y, u) \in E_{1+s+m}$ with $(t, y) \in A_{0}, u \in U(t, y)$, and let $f(t, y, u)=\left(f_{1}, \cdots, f_{n}\right)$ be a continuous: vector function from $M_{0}$ into $E_{n}$. Let $Q(t, y)=f(t, y, U(t, y))$ be a closed convex subset of $E_{n}$ for every $(t, y) \in A_{0}$. Assume that $U(t, y)$ has property $(U)$ in $A_{0}$ and that $Q(t, y)$ has property $(Q)$ in $A_{0}$. Let $x_{k}(t), t_{1 k} \leqq t \leqq t_{2 k}, k=1,2, \cdots$, be a sequence of trajectories, $x_{k}(t)=\left(y_{k}(t), z_{k}(t)\right)$, for which we assume that the s-vector $y_{k}(t)$ converges in the $\rho$-metric toward an $A C$ vector function $y(t), t_{1} \leqq t \leqq t_{2}$, and that the $(n-s)$-vector $z_{k}(t)$ converges pointwise for almost all $t_{1}<t<t_{2}$, toward a vector $z(t)$ which admits of a decomposition $z(t)=Z(t)+S(t)$ where $Z(t)$ is an $A C$ vector function in $\left[t_{1}, t_{2}\right]$, and $S^{\prime}(t)=0$ a.e. in $\left[t_{1}, t_{2}\right]$ (that is, $S(t)$ is a singularfunction). Then, the $A C$ vector $X(t)=[y(t), Z(t)], t_{1} \leqq t \leqq t_{2}$, is a trajectory.

Remark. For $s=n$, this theorem reduces to Closure Theorem I.

Proof of Closure Theorem II. The vector functions

$$
\begin{gathered}
\phi(t)=X^{\prime}(t)=\left(y^{\prime}(t), Z^{\prime}(t)\right), \quad t_{1} \leqq t \leqq t_{2}, \\
\phi_{k}(t)=x_{k}^{\prime}(t)=\left(y_{k}^{\prime}(t), z_{k}^{\prime}(t)\right)=f\left(t, y_{k}(t), u_{k}(t)\right), \quad t_{1 k} \leqq t \leqq t_{2 k}, \quad k=1,2, \cdots,
\end{gathered}
$$

are defined almost everywhere and are $L$-integrable. We have to prove that $[t, y(t), Z(t)] \in A$ for every $t_{1} \leqq t \leqq t_{2}$, and that there is a measurable control function $u(t), t_{1} \leqq t \leqq t_{2}$, such that 


$$
\begin{gathered}
\phi(t)=X^{\prime}(t)=\left(y^{\prime}(t), Z^{\prime}(t)\right)=f(t, y(t), u(t)), \\
u(t) \in U(t, y(t)),
\end{gathered}
$$

for almost all $t \in\left[t_{1}, t_{2}\right]$.

First, $\rho\left(y_{k}, y\right) \rightarrow 0$ as $k \rightarrow 0$; hence $t_{1 k} \rightarrow t_{1}, t_{2 k} \rightarrow t_{2}$. If $t \in\left(t_{1}, t_{2}\right)$, or $t_{1}<t<t_{2}$, then $t_{1 k}<t<t_{2 k}$ for all $k$ sufficiently large, and $\left(t, y_{k}(t)\right) \in A_{0}$. Since $y_{k}(t) \rightarrow y(t)$ as $k \rightarrow \infty$ and $A_{0}$ is closed, we conclude that $(t, y(t)) \in A_{0}$ for every $t_{1}<t<t_{2}$, and finally $(t, y(t), Z(t)) \in A_{0} \times E_{n-s}$, or $(t, X(t)) \in A, t_{1} \leqq t \leqq t_{2}$.

For almost all $t \in\left[t_{1}, t_{2}\right]$ the derivative $X^{\prime}(t)=\left[y^{\prime}(t), Z^{\prime}(t)\right]$ exists and is finite, $S^{\prime}(t)$ exists and $S^{\prime}(t)=0$, and $z_{k}(t) \rightarrow z(t)$. Let $t_{0}$ be such a point with $t_{1}<t_{0}<t_{2}$. Then there is a $\sigma>0$ with $t_{1}<t_{0}-\sigma<t_{0}+\sigma<t_{2}$, and, for some $k_{0}$ and all $k \geqq k_{0}$, also $t_{1 k}<t_{0}-\sigma<t_{0}+\sigma<t_{2 k}$. Let $x_{0}=X\left(t_{0}\right)=\left(y_{0}, Z_{0}\right)$, or $y_{0}=y\left(t_{0}\right)$, $Z_{0}=Z\left(t_{0}\right)$. Let $z_{0}=z\left(t_{0}\right), S_{0}=S\left(t_{0}\right)$. We have $S^{\prime}\left(t_{0}\right)=0$, hence $z^{\prime}\left(t_{0}\right)$ exists and $z^{\prime}\left(t_{0}\right)=Z^{\prime}\left(t_{0}\right)$. Also, we have $z_{k}\left(t_{0}\right) \rightarrow z\left(t_{0}\right)$.

We have $y_{k}(t) \rightarrow y(t)$ uniformly in $\left[t_{0}-\sigma, t_{0}+\sigma\right]$, and all functions $y(t), y_{k}(t)$ are continuous in the same interval. Thus, they are equicontinuous in $\left[t_{0}-\sigma, t_{0}+\sigma\right]$. Given $\varepsilon>0$, there is a $\delta>0$ such that $t, t^{\prime} \in\left[t_{0}-\sigma, t_{0}+\sigma\right]$, $\left|t-t^{\prime}\right| \leqq \delta, k \geqq k_{0}$, implies

$$
\left|y(t)-y\left(t^{\prime}\right)\right| \leqq \varepsilon / 2, \quad\left|y_{k}(t)-y_{k}\left(t^{\prime}\right)\right| \leqq \varepsilon / 2 .
$$

We can assume $0<\delta<\sigma, \delta \leqq \varepsilon$. For any $h, 0<h \leqq \delta$, let us consider the averages

$$
\begin{aligned}
& m_{h}=h^{-1} \int_{0}^{h} \phi\left(t_{0}+s\right) d s=h^{-1}\left[X\left(t_{0}+h\right)-X\left(t_{0}\right)\right], \\
& m_{h k}=h^{-1} \int_{0}^{h} \phi_{k}\left(t_{0}+s\right) d s=h^{-1}\left[x_{k}\left(t_{0}+h\right)-x_{k}\left(t_{0}\right)\right],
\end{aligned}
$$

where $X=(y, Z), x_{k}=\left(y_{k}, z_{k}\right)$.

Given $\eta>0$ arbitrary, we can fix $h, 0<h \leqq \delta<\sigma$, so small that

$$
\begin{gathered}
\left|m_{h}-\phi\left(t_{0}\right)\right| \leqq \eta, \\
\left|S\left(t_{0}+h\right)-S\left(t_{0}\right)\right|<\eta h / 4 .
\end{gathered}
$$

This is possible since $h^{-1} \int_{0}^{h} \phi\left(t_{0}+s\right) d s \rightarrow \phi\left(t_{0}\right)$ and $\left[S\left(t_{0}+h\right)-S\left(t_{0}\right)\right] h^{-1}$ $\rightarrow 0$ as $h \rightarrow 0+$. Also, we can choose $h$ in such a way that $z_{k}\left(t_{0}+h\right) \rightarrow z\left(t_{0}+h\right)$ as $k \rightarrow+\infty$. This is possible since $z_{k}(t) \rightarrow z(t)$ for almost all $t_{1}<t<t_{2}$.

Having so fixed $h$, let us take $k_{1} \geqq k_{0}$ so large that

$$
\begin{aligned}
& \left|y_{k}\left(t_{0}\right)-y\left(t_{0}\right)\right|,\left|y_{k}\left(t_{0}+h\right)-y\left(t_{0}+h\right)\right| \leqq \min [\eta h / 4, \varepsilon / 2], \\
& \left|z_{k}\left(t_{0}\right)-z\left(t_{0}\right)\right|,\left|z_{k}\left(t_{0}+h\right)-z\left(t_{0}+h\right)\right| \leqq \eta h / 8 .
\end{aligned}
$$

This is possible since $y_{k}(t) \rightarrow y(t), z_{k}(t) \rightarrow z(t)$ both at $t=t_{0}$ and $t=t_{0}+h$. Then we have 


$$
\begin{aligned}
\mid h^{-1}\left[y _ { k } \left(t_{0}\right.\right. & \left.+h)-y_{k}\left(t_{0}\right)\right]-h^{-1}\left[y\left(t_{0}+h\right)-y\left(t_{0}\right)\right] \mid \\
& \leqq\left|h^{-1}\left[y_{k}\left(t_{0}+h\right)-y\left(t_{0}+h\right)\right]\right|+\left|h^{-1}\left[y_{k}\left(t_{0}\right)-y\left(t_{0}\right)\right]\right| \\
& \leqq h^{-1}(\eta h / 4)+h^{-1}(\eta h / 4)=\eta / 2 .
\end{aligned}
$$

Analogously, since $z=Z+S$, we have

$$
\begin{aligned}
& \left|h^{-1}\left[z_{k}\left(t_{0}+h\right)-z_{k}\left(t_{0}\right)\right]-h^{-1}\left[Z\left(t_{0}+h\right)-Z\left(t_{0}\right)\right]\right| \\
& \left.=\mid h^{-1}\left[z_{k}\left(t_{0}+h\right)-z_{k}\left(t_{0}\right)\right]-h^{-1}\left[z\left(t_{0}+h\right)-z\left(t_{0}\right)\right]+h^{-1}\left[S\left(t_{0}+h\right)-S\left(t_{0}\right)\right)\right] \mid \\
& \leqq\left|h^{-1}\left[z_{k}\left(t_{0}+h\right)-z\left(t_{0}+h\right)\right]\right|+\left|h^{-1}\left[z_{k}\left(t_{0}\right)-z\left(t_{0}\right)\right]\right|+\left|h^{-1}\left[S\left(t_{0}+h\right)-S\left(t_{0}\right)\right]\right| \\
& \leqq h^{-1}(\eta h / 8)+h^{-1}(\eta h / 8)+h^{-1}(\eta h / 4)=\eta / 2 .
\end{aligned}
$$

Finally, we have

$$
\begin{aligned}
\left|m_{h k}-m_{h}\right|= & \left|h^{-1}\left[x_{k}\left(t_{0}+h\right)-x_{k}\left(t_{0}\right)\right]-h^{-1}\left[X\left(t_{0}+h\right)-X\left(t_{0}\right)\right]\right| \\
\leqq & \left|h^{-1}\left[y_{k}\left(t_{0}+h\right)-y_{k}\left(t_{0}\right)\right]-h^{-1}\left[y\left(t_{0}+h\right)-y\left(t_{0}\right)\right]\right| \\
& +\left|h^{-1}\left[z_{k}\left(t_{0}+h\right)-z_{k}\left(t_{0}\right)\right]-h^{-1}\left[Z\left(t_{0}+h\right)-Z\left(t_{0}\right)\right]\right| \\
\leqq & \eta / 2+\eta / 2=\eta .
\end{aligned}
$$

We conclude that, for the chosen value of $h, 0<h \leqq \delta<\sigma$, and every $k \geqq k_{1}$, we have

$$
\left|m_{h}-\phi\left(t_{0}\right)\right| \leqq \eta,\left|m_{h k}-m_{h}\right| \leqq \eta,\left|y_{k}\left(t_{0}\right)-y\left(t_{0}\right)\right| \leqq \varepsilon / 2 .
$$

For $0 \leqq s \leqq h$ we have now

$$
\begin{gathered}
\left|y_{k}\left(t_{0}+s\right)-y\left(t_{0}\right)\right| \leqq\left|y_{k}\left(t_{0}+s\right)-y_{k}\left(t_{0}\right)\right|+\left|y_{k}\left(t_{0}\right)-y\left(t_{0}\right)\right| \leqq \varepsilon / 2+\varepsilon / 2=\varepsilon, \\
\left|\left(t_{0}+s\right)-t_{0}\right| \leqq h \leqq \delta \leqq \varepsilon, \\
f\left(t_{0}+s, y_{k}\left(t_{0}+s\right), u_{k}\left(t_{0}+s\right)\right) \in Q\left(t_{0}+s, y_{k}\left(t_{0}+s\right)\right) .
\end{gathered}
$$

Hence, by definition of $Q\left(t_{0}, y_{0}, 2 \varepsilon\right)$, also

$$
\phi_{k}\left(t_{0}+s\right)=f\left(t_{0}+s, y_{k}\left(t_{0}+s\right), u_{k}\left(t_{0}+s\right)\right) \in Q\left(t_{0}, y_{0}, 2 \varepsilon\right) .
$$

The second integral relation (11) shows that we have also

$$
m_{h k} \in \operatorname{clco} Q\left(t_{0}, y_{0}, 2 \varepsilon\right)
$$

since the latter is a closed convex set. Finally, by relations (12), we deduce

$$
\left|\phi\left(t_{0}\right)-m_{h k}\right| \leqq\left|\phi\left(t_{0}\right)-m_{h}\right|+\left|m_{h}-m_{h k}\right| \leqq 2 \eta,
$$

and hence 


$$
\phi\left(t_{0}\right) \in\left[\operatorname{clco} Q\left(t_{0}, y_{0}, 2 \varepsilon\right)\right]_{2 \eta} .
$$

Here $\eta>0$ is an arbitrary number, and the set in brackets is closed. Hence

$$
\phi\left(t_{0}\right) \in \operatorname{cl} \text { co } Q\left(t_{0}, y_{0}, 2 \varepsilon\right),
$$

and this relation holds for every $\varepsilon>0$. By property $(Q)$ we have

$$
\phi\left(t_{0}\right) \in \bigcap_{\varepsilon} \operatorname{clco} Q\left(t_{0}, y_{0}, 2 \varepsilon\right)=Q\left(t_{0}, y_{0}\right),
$$

where $y_{0}=y\left(t_{0}\right)$, and $Q\left(t_{0}, y_{0}\right)=f\left(t_{0}, y_{0}, U\left(t_{0}, y_{0}\right)\right)$. This relation implies that there are points $\bar{u}=\bar{u}\left(t_{0}\right) \in U\left(t_{0}, y_{0}\right)$ such that

$$
\phi\left(t_{0}\right)=f\left(t_{0}, y\left(t_{0}\right), \bar{u}\left(t_{0}\right)\right) .
$$

This holds for almost all $t_{0} \in\left[t_{1}, t_{2}\right]$. Hence, there is at least one function $\bar{u}(t)$, defined a.e. in $\left[t_{1}, t_{2}\right]$, for which relation (10) holds a.e. in $\left[t_{1}, t_{2}\right]$. We have to prove that there is at least one such function which is measurable. The proof is exactly as the one for Closure Theorem $\mathrm{I}$, where we write $y, y_{k}$ instead of $x, x_{k}$, and will not be repeated here. Closure Theorem II is thereby proved.

6. Notations for Lagrange problems with unilateral constraints. Let $A$ be a closed set of the $(t, x)$-space $E_{1} \times E_{n}$, and, for every $(t, x) \in A$, let $U(t, x)$ be a given subset of $E_{m}$. Let $f_{i}(t, x, u), i=0,1, \cdots, n$, be real-valued continuous functions in the set $M \subset E_{1} \times E_{n} \times E_{m}$ of all $(t, x, u)$ with $u \in U(t, x),(t, x) \in A$. Let $f$ and $\bar{f}$ be the $n$-dim and $(n+1)$-dim vector functions

$$
f=\left(f_{1}, \cdots, f_{n}\right), \quad \tilde{f}=\left(f_{0}, f_{1}, \cdots, f_{n}\right) .
$$

As usual we say that $u(t)=\left(u^{1}, \cdots, u^{m}\right), x(t)=\left(x^{1}, \cdots, x^{n}\right), t_{1}<t<t_{2}$, is an admissible pair provided (a) $u(t)$ is measurable in $\left[t_{1}, t_{2}\right]$; (b) $x(t)$ is AC in $\left[t_{1}, t_{2}\right]$, (c) $[t, x(t)] \in A$ for every $t \in\left[t_{1}, t_{2}\right] ;$ (d) $u(t) \in U(t, x(t))$ a.e. in $\left[t_{1}, t_{2}\right]$; (e) $f_{i}(t, x(t), u(t))$ is $L$-integrable in $\left[t_{1}, t_{2}\right], i=0,1, \cdots, n$, and $d x^{i} / d t=f_{i}(t, x(t), u(t)$, $i=1, \cdots, n$, a.e. in $\left[t_{1}, t_{2}\right]$. Thus, by introducing the auxiliary variable $x^{0}$, the differential equation $d x^{0} / d t=f_{0}(t, x(t), u(t))$, the boundary condition $x^{0}\left(t_{1}\right)=0$, the vector $\tilde{x}=\left(x^{0}, x^{1}, \cdots, x^{n}\right)$, and the set $\left(\tilde{A}=A \times E_{1} \subset E_{n+2}\right)$, the pair $[u(t), x(t)]$ is admissible if and only if the pair $[u(t), \tilde{x}(t)]$ is admissible according to the definitions of no. 2 for the set $\bar{A}$ of the $t \tilde{x}$-space $E_{1} \times E_{n+1}$, the sets $U(t, x) \subset E_{m}$, and the vector function $\tilde{f}(t, x, u)$.

If $[u(t), x(t)]$ is admissible, then $u(t)$ is said to be an admissible control function, $x(t)$ a trajectory, and

$$
x^{0}\left(t_{2}\right)=I[x, u]=\int_{t_{1}}^{t_{2}} f_{0}(t, x(t), u(t)) d t
$$

the cost functional.

A class $\Omega$ of admissible pairs $x(t), u(t)$ is said to be complete if for every sequence $x_{k}(t), u_{k}(t), t_{1 k} \leqq t \leqq t_{2 k}, k=1,2, \cdots$, of admissible pairs all in $\Omega$, with the sequence 
$\left[x_{k}(t)\right]$ converging in the metric $\rho$ toward a vector function $x(t)$ which is known to be a trajectory generated by some admissible control function $u(t)$, then $[x(t), u(t)]$ belongs to $\Omega$.

Complete classes $\Omega$ are often defined in terms of boundary conditions. For instance, if $B$ is a given closed set of points $\left(t_{1}, x_{1}, t_{2}, x_{2}\right)$ of the $(2 n+2)$-dim Euclidean space $E_{2 n+2}$, we may define $\Omega$ as the class of all admissible pairs $x(t)$, $u(t)$ satisfying

$$
\left(t_{1}, x\left(t_{1}\right), t_{2}, x\left(t_{2}\right)\right) \in B
$$

Then $\Omega$ is a complete class in the sense mentioned above, since $B$ is, by hypothesis, a closed set.

We shall denote by $B_{1}$ the projection of $B$ on the $\left(t, x_{1}\right)$-space $E_{n+1}$, that is, $B_{1}$ is the set of all points $\left(t_{1}, x_{1}\right) \in E_{n+1}$ for $\left(t_{1}, x_{1}, t_{2}, x_{2}\right) \in B$. Analogously, we denote by $B_{2}$ the projection of $B$ on the $\left(t_{2}, x_{2}\right)$-space $E_{n+1}$. Obviously, $B \subset B_{1}$ $\times B_{2}$, and $B_{1} \times B_{2}$ may be larger than $B$.

It is often requested that each trajectory $x(t)$ of a class $\Omega$ as above possesses at least one point $\left(t^{*}, x\left(t^{*}\right)\right)$ on a given compact subset $P$ of $A$. Such a condition is certainly satisfied if $B$ is compact, or at least if $B$ is closed and $B_{1}$, or $B_{2}$, is compact.

For the analysis of problems of Lagrange with unilateral constraints certain variable sets have to be taken into consideration, namely, the set $U(t, x)$ above and the sets

$$
\begin{aligned}
Q(t, x) & =[z \mid z=f(t, x, u), u \in U(t, x)]=f[t, x, U(t, x)] \subset E_{n}, \\
\tilde{Q}(t, x) & =[\tilde{z} \mid \tilde{z}=\tilde{f}(t, x, u), u \in U(t, x)]=\tilde{f}[t, x, U(t, x)] \\
& =\left[\tilde{z}=\left(z^{0}, z\right) \mid z^{0}=f_{0}(t, x, u), z=f(t, x, u), u \in U(t, x)\right] \subset E_{n+1}, \\
\tilde{Q}(t, x) & =\left[\tilde{z}=\left(z^{0}, z\right) \mid z^{0} \geqq f_{0}(t, x, u), z=f(t, x, u), u \in U(t, x)\right] \subset E_{n+1} .
\end{aligned}
$$

The sets $Q$ and $\widetilde{Q}$ are well known and have been considered by a number of authors (for instance, A. F. Filippov [2]). The set $\widetilde{\tilde{Q}}(t, x)$ is being considered here and in [1c] for the first time. By considering this set, instead of $Q$ or $\tilde{Q}$, we prove in $\S \S 7,9$ Theorems I and II which include a number of existence theorems for both problems of optimal control and the calculus of variations.

\section{An existence theorem for Lagrange problems with unilateral constraints.}

EXISTENCE THEOREM I. Let $A$ be a compact subset of the tx-space $E_{1} \times E_{n}$, and for every $(t, x) \in A$ let $U(t, x)$ be a closed subset of the $u$-space $E_{m}$. Let $\tilde{f}(t, x, u)$ $=\left(f_{0}, f_{1}, \cdots, f_{n}\right)=\left(f_{0}, f\right)$ be a continuous vector function on the set $M$ of all $(t, x, u)$ with $(t, x) \in A, u \in U(t, x)$. Assume that, for every $(t, x) \in A$ the set

$$
\tilde{\tilde{Q}}(t, x)=\left[\tilde{z}=\left(z^{0}, z\right) \mid z^{0} \geqq f_{0}(t, x, u), z=f(t, x, u), u \in U(t, x)\right] \subset E_{n+1}
$$

is convex. Assume that $U(t, x)$ satisfies property $(U)$ in $A$, and $\tilde{Q}(t, x)$ satisfies 
property $(Q)$ in $A$. Assume that there is a continuous scalar function $\Phi(\zeta), 0 \leqq \zeta$ $<+\infty$, with $\Phi(\zeta) / \zeta \rightarrow+\infty$ as $\zeta \rightarrow+\infty$, such that $f_{0}(t, x, u) \geqq \Phi(|u|)$ for all $(t, x, u) \in M$, and that there are constants $C, D \geqq 0$ such that $|f(t, x, u)| \leqq C+D|u|$ for all $(t, x, u) \in M$. Then the cost functional $I[x, u]=\int_{t_{1}}^{t_{2}} f_{0}(t, x, u) d t$ has an absolute minimum in any nonempty complete class $\Omega$ of admissible pairs $x(t), u(t)$.

If $A$ is not compact, but closed and contained in a slab $\left[t_{0} \leqq t \leqq T, x \in E_{n}\right], t_{0}, T$ finite, then Theorem I still holds if, in addition, we know that (a)

$$
x^{1} f_{1}+\cdots+x^{n} f_{n} \leqq F\left[|x|^{2}+1\right]
$$

for all $(t, x, u) \in M$ and some constant $F \geqq 0$, and (b) every trajectory in $\Omega$ contains at least one point $\left(t^{*}, x\left(t^{*}\right)\right)$ on a given compact subset $P$ of $A$ ( $t^{*}$ may depend on $x(t)$ ). If $A$ is not compact, nor contained in a slab as above, but $A$ is closed, then Theorem I still holds if hypotheses (a), (b) are satisfied, and (c) $f_{0}(t, x, u)$ $\geqq \mu>0$ for all $(t, x, u) \in M$ with $|t| \geqq R$, for convenient constants $\mu>0, R \geqq 0$. Finally condition (a) can be replaced in either case by the hypotheses: ( $a^{\prime}$ ) There are constants $G>0, H \geqq 0$ such that $f_{0}(t, x, u) \geqq G|f(t, x, u)|$ for all $(t, x, u) \in M$ with $|x| \geqq H$. Furthermore, when $A$ is not compact but closed, both conditions $f_{0} \geqq \Phi(|u|),|f| \leqq C+D|u|$ can be replaced by the following condition: $(\gamma)$ for every compact subset $A_{0}$ of $A$ there is a function $\Phi_{0}$ as above and constants $C_{0}, D_{0} \geqq 0$ such that $f_{0} \geqq \Phi_{0}(|u|),|f| \leqq C_{0}+D_{0}|u|$ for all $(t, x, u) \in M$ with $(t, x) \in A_{0}$ (where $\Phi_{0}, C_{0}, D_{0}$ may depend on $A_{0}$ ).

Proof of Existence Theorem I. We have $\Phi(\zeta) \geqq-M_{0}$ for some number $M_{0} \geqq 0$, hence $\Phi(\zeta)+M_{0} \geqq 0$ for all $\zeta \geqq 0$, and $f_{0}(t, x, u)+M_{0} \geqq 0$ for all $(t, x, u) \in M$. Let $D$ be the diameter of $A$. Then for every pair $x(t), u(t), t_{1} \leqq t \leqq t_{2}$, of $\Omega$ we have

$$
I[x, u]=\int_{t_{1}}^{t_{2}} f_{0} d t \geqq \int_{t_{1}}^{t_{2}} \Phi(|u|) d t \geqq-D M_{0}>-\infty .
$$

Let $i=\operatorname{Inf} I[x, u]$, where $\operatorname{Inf}$ is taken for all pairs $(x, u) \in \Omega$. Then $i$ is finite.

Let $x_{k}(t), u_{k}(t), t_{1 k} \leqq t \leqq t_{2 k}, k=1,2, \cdots$, be a sequence of admissible pairs all in $\Omega$, such that $I\left[x_{k}, u_{k}\right] \rightarrow i$ as $k \rightarrow \infty$. We may assume

$$
i \leqq I\left[x_{k}, u_{k}\right]=\int_{t_{1 k}}^{t_{2 k}} f_{0}\left(t, x_{k}(t), u_{k}(t)\right) d t \leqq i+k^{-1} \leqq i+1, \quad k=1,2, \cdots
$$

Since $A$ is compact, the sequence $\left[x_{k}(t)\right]$ is equibounded.

Let us prove that the AC vector functions $x_{k}(t), t_{1 k} \leqq t \leqq t_{2 k}, k=1,2, \cdots$, are equiabsolutely continuous. Let $\varepsilon>0$ be any given number, and let $\sigma=2^{-1} \varepsilon\left(D M_{0}+|i|+1\right)^{-1}$. Let $N>0$ be a number such that $\Phi(z) / z>1 / \sigma$ for $z \geqq N$. Let $E$ be any measurable subset of $\left[t_{1 k}, t_{2 k}\right]$ with meas $E<\eta=\varepsilon / 2 N$. Let $E_{1}$ be the subset of all $t \in E$ where $u_{k}(t)$ is finite and $\left|u_{k}(t)\right| \leqq N$, and let 
$E_{2}=E-E_{1}$. Then $\left|u_{k}(t)\right| \leqq N$ in $E_{1}$, and $\Phi\left(\left|u_{k}\right|\right) /\left|u_{k}\right| \geqq 1 / \sigma$, or $u_{k} \leqq \sigma \Phi\left(\left|u_{k}\right|\right)$, a.e. in $E_{2}$. Hence

$$
\begin{aligned}
\int_{E}\left|u_{k}(t)\right| d t & =\left(\int_{E_{1}}+\int_{E_{2}}\right)\left|u_{k}(t)\right| d t \\
& \leqq N \text { meas } E_{1}+\sigma \int_{E_{2}} \Phi\left(\left|u_{k}(t)\right|\right) d t \\
& \leqq N \text { meas } E+\sigma \int_{E_{2}}\left[\Phi\left(\left|u_{k}(t)\right|\right)+M_{0}\right] d t \\
& \leqq N \eta+\sigma \int_{t_{1 k}}^{t_{2 k}}\left[\Phi\left(\left|u_{k}(t)\right|\right)+M_{0}\right] d t \\
& \leqq N \eta+\sigma \int_{t_{1 k}}^{t_{2 k}}\left[f_{0}\left(t, x_{k}(t), u_{k}(t)\right)+M_{0}\right] d t \\
& \leqq N \eta+\sigma\left(D M_{0}+|i|+1\right) \\
& \leqq \varepsilon / 2+\varepsilon / 2=\varepsilon .
\end{aligned}
$$

This proves that the vector functions $u_{k}(t), t_{1 k} \leqq t \leqq t_{2 k}, k=1,2, \cdots$, are equiabsolutely integrable. From here we deduce

$$
\begin{aligned}
\int_{E}\left(\mid x_{k}^{\prime} t\right) \mid d t & =\int_{E}\left|f\left(t, x_{k}(t), u_{k}(t)\right)\right| d t \leqq \int_{E}\left[A+B\left|u_{k}(t)\right|\right] d t \\
& \leqq A \text { meas } E+B \int_{E}\left|u_{k}(t)\right| d t
\end{aligned}
$$

and this proves the equiabsolute continuity of the vector functions $x_{k}(t)$, $t_{1 k} \leqq t \leqq t_{2 k}, k=1,2, \cdots$.

Now let us consider the sequence of AC scalar functions $x_{k}^{0}(t)$ defined by

$$
x_{k}^{0}(t)=\int_{t_{1 k}}^{t} f_{0}\left(\tau, x_{k}(\tau), u_{k}(\tau)\right) d t, \quad t_{1 k} \leqq t \leqq t_{2 k},
$$

with

$$
\begin{gathered}
x_{k}^{0}\left(t_{1 k}\right)=0, x_{k}^{0}\left(t_{2 k}\right)=I\left[x_{k}, u_{k}\right] \rightarrow i \text { as } k \rightarrow+\infty, \\
i \leqq x_{k}^{0}\left(t_{2 k}\right) \leqq i+k^{-1} \leqq i+1
\end{gathered}
$$

If $u_{k}^{0}(t)=f_{0}\left(t, x_{k}(t), u_{k}(t)\right), t_{1 k} \leqq t \leqq t_{2 k}$, then we define the functions $u_{k}^{-}(t), u_{k}^{+}(t)$ as follows:

$$
u_{k}^{-}(t)=-M_{0}, \quad u_{k}^{+}(t)=u_{k}^{0}(t)+M_{0}, \quad t_{1 k} \leqq t \leqq t_{2 k} .
$$

Then $u_{k}^{-}(t) \leqq 0, u_{k}^{+}(t) \geqq 0$ a.e. in $\left[t_{1 k}, t_{2 k}\right]$, and we define 


$$
y_{k}^{-}(t)=\int_{t_{1 k}}^{t} u^{-}(t) d t, \quad y_{k}^{+}(t)=\int_{t_{1 k}}^{t} u^{+}(t) d t, \quad t_{1 k} \leqq t \leqq t_{2 k}, k=1,2, \ldots
$$

Since $-M_{0}=u_{k}^{-}(t) \leqq 0$, we have $-M_{0}\left(t-t_{1 k}\right)=y_{k}^{-}(t) \leqq 0$, and the functions $y_{k}^{-}(t)$ are monotone nonincreasing and uniformly Lipschitzian with constant $M_{0}$. On the other hand, the functions $y_{k}^{+}(t)$ are nonnegative, monotone nondecreasing, and uniformly bounded since

$$
\begin{aligned}
0 \leqq y_{k}^{+}\left(t_{2 k}\right) & =\left(y_{k}^{+}\left(t_{2 k}\right)+y_{k}^{-}\left(t_{2 k}\right)\right)-y_{k}^{-}\left(t_{2 k}\right)=x_{k}^{0}\left(t_{2 k}\right)-y_{k}^{-}\left(t_{2 k}\right) \\
& \leqq i+1+M_{0}\left(t_{2 k}-t_{1 k}\right) \leqq D M_{0}+|i|+1 .
\end{aligned}
$$

By Ascoli's theorem we first extract a sequence for which $x_{k}(t), y_{k}^{-}(t), t_{1 k} \leqq t$ $\leqq t_{2 k}$, converges in the metric $\rho$ toward a continuous vector function $x(t), Y^{-}(t)$, $t_{1} \leqq t \leqq t_{2}$. Here $x(t)$ is $A C$ because of the equiabsolute continuity of the vector functions $x_{k}(t)$, and $Y^{-}(t)=-M_{0}\left(t-t_{1}\right), Y^{-}\left(t_{1}\right)=0$. Then we apply Helly's theorem to the sequence $y_{k}^{+}(t)$ and we perform a successive extraction so that the corresponding sequence of the $y_{k}^{+}(t)$ converges for every $t_{1}<t<t_{2}$ toward a function $Y_{0}^{+}(t), t_{1}<t<t_{2}$, which is nonnegative, monotone nondecreasing, but not necessarily continuous. We define $Y_{0}^{+}(t)$ at $t_{1}$ by taking $Y_{0}^{+}\left(t_{1}\right)=0$, and at $t_{2}$ by continuity at $t_{2}$, because of its monotoneity. Thus $0 \leqq Y_{0}^{+}(t) \leqq D M_{0}+i+1, t_{1}$ $\leqq t \leqq t_{2}$.

Finally, $Y_{0}^{+}(t)$ admits of a unique decomposition $Y_{0}^{+}(t)=Y^{+}(t)+Z(t)$, $t_{1} \leqq t \leqq t_{2}$, with $Y^{+}\left(t_{1}\right)=0$, where both $Y^{+}(t), Z(t)$ are nonnegative monotone, nondecreasing, where $Y^{+}(t)$ is $\mathrm{AC}$, and $Z^{\prime}(t)=0$ a.e. in $\left[t_{1}, t_{2}\right]$. Finally, if $Y(t)$ $=Y^{-}(t)+Y^{+}(t)$, we see that $x_{k}(t), t_{1} \leqq t \leqq t_{2}$, converges for all $t_{1}<t<t_{2}$ toward $Y(t)+Z(t)$, where $Y(t)$ is a (scalar) AC function, $-D M_{0} \leqq Y(t) \leqq D M_{0}$ $+|i|+1, Y\left(t_{1}\right)=0$. Let us prove that $Y\left(t_{2}\right) \leqq i$. For the subsequence [k] we have extracted last, we have $t_{2 k} \rightarrow t_{2}, x_{k}^{0}\left(t_{2 k}\right) \rightarrow i, x_{k}^{0}\left(t_{2 k}\right)=y_{k}^{-}\left(t_{2 k}\right)+y_{k}^{+}\left(t_{2 k}\right)$. If $\bar{t}_{2}$ is any point, $t_{1}<\bar{t}_{2}<t_{2}, \bar{t}_{2}$ as close as we want to $t_{2}$, then $\bar{t}_{2}<t_{2 k}$ for all $k$ sufficiently large (of the extracted sequence), since $t_{2 k} \rightarrow t_{2}$. We can assume $k$ so large that $\bar{t}_{2}<t_{2 k},\left|\bar{t}_{2}-t_{2 k}\right|<2\left|\bar{t}_{2}-t_{2}\right|$. Then

$$
\left|\bar{y}_{k}^{-}\left(\bar{t}_{2}\right)-\bar{y}_{k}^{-}\left(t_{2 k}\right)\right|=M_{0}\left|\bar{t}_{2}-t_{2 k}\right| \leqq 2 M_{0}\left|\bar{t}_{2}-t_{2}\right| \text {. }
$$

Since $y_{k}(t)^{+}$is nondecreasing, we have $y_{k}^{+}\left(\bar{t}_{2}\right) \leqq y_{k}^{+}\left(t_{2 k}\right)$, and finally

$$
\begin{aligned}
y_{k}^{-}\left(\bar{t}_{2}\right)+y_{k}^{+}\left(\bar{t}_{2}\right) & \leqq y_{k}^{-}\left(\bar{t}_{2}\right)+y_{k}^{+}\left(t_{2 k}\right) \\
& \leqq y_{k}^{-}\left(t_{2 k}\right)+y_{k}^{+}\left(t_{2 k}\right)+\left|y_{k}^{-}\left(\bar{t}_{2}\right)-y_{k}^{-}\left(t_{2 k}\right)\right| \\
& \leqq x_{k}^{0}(t)_{2 k}+2 M_{0}\left|\bar{t}_{2}-t_{2}\right|
\end{aligned}
$$

where $x^{0}\left(t_{2 k}\right) \rightarrow i$ as $k \rightarrow+\infty$, and $x_{k}^{0}\left(t_{2 k}\right)<i+k^{-1}$. Hence

$$
y_{k}^{-}\left(\bar{t}_{2}\right)+y_{k}^{+}\left(\bar{t}_{2}\right)<i+2 M_{0}\left|\bar{t}_{2}-t_{2}\right|+k^{-1} \text {. }
$$


As $k \rightarrow+\infty$ (along the extracted sequence), we have

or

$$
Y^{-}\left(\bar{t}_{2}\right)+Y_{0}^{+}\left(\bar{t}_{2}\right) \leqq i+2 M_{0}\left|\bar{t}_{2}-t_{2}\right|
$$

$$
Y^{-}\left(\bar{t}_{2}\right)+Y^{+}\left(\bar{t}_{2}\right)+Z\left(\bar{t}_{2}\right) \leqq i+2 M_{0}\left|\bar{t}_{2}-t_{2}\right|
$$

where the third term in the first member is $\geqq 0$. Thus

$$
Y\left(\bar{t}_{2}\right)=Y^{-}\left(\bar{t}_{2}\right)+Y^{+}\left(\bar{t}_{2}\right) \leqq i+2 M_{0}\left|\bar{t}_{2}-t_{2}\right| \text {. }
$$

As $\bar{t}_{2} \rightarrow t_{2}-0$, we obtain $Y\left(t_{2}\right) \leqq i$, since $Y$ is continuous at $t_{2}$.

We will apply below Closure Theorem II to an auxiliary problem we shall now define. Let $\tilde{u}=\left(u^{0}, u\right)=\left(u^{0}, u^{1}, \cdots, u^{m}\right)$, let $\tilde{U}(t, x)$ be the set of all $\tilde{u} \in E_{m+1}$ with $u=\left(u^{1}, \cdots, u^{m}\right) \in U(t, x), u^{0} \geqq f_{0}(t, x, u)$, let $\tilde{x}=\left(x^{0}, x\right)=\left(x^{0}, x^{1}, \cdots, x^{n}\right)$, let $\widetilde{\widetilde{f}}=\tilde{\tilde{f}}(t, x, u)=\left(\tilde{f}_{0}, f\right)=\left(\tilde{f}_{0}, f_{1}, \cdots, f_{n}\right)$ with $\tilde{f}_{0}=u^{0}$. Thus $\tilde{\tilde{f}}$ depends only on $t, x, \tilde{u}$ (instead of $t, \tilde{x}, \tilde{u})$, and $U$ depends only on $t, x$, (instead of $t, \tilde{x})$. Finally we consider the differential system

or

$$
d \tilde{x} / d t=\tilde{\tilde{f}}(t, x, u)
$$

$$
d x^{0} / d t=u^{0}(t), \quad d x^{i} / d t=f_{i}(t, x, u), \quad i=1, \cdots, n,
$$

with constraints

$$
\tilde{u}(t) \in \tilde{U}(t, x(t))
$$

or

$$
u^{0}(t) \geqq f_{0}(t, x(t), u(t)), \quad u(t) \in U(t, x(t)),
$$

a.e. in $\left[t_{1}, t_{2}\right]$, besides $x^{0}\left(t_{1}\right)=0$, and $[x, u] \in \Omega$. We have here the situation discussed in Closure Theorem II where $\tilde{x}$ replaces $x, x$ replaces $y, x^{0}$ replaces $z, n+1$ replaces $n, n$ replaces $s$, hence $(n+1)-n=1$ replaces $n-s$. For the new auxiliary problem the cost functional is

$$
J[\tilde{x}, \tilde{u}]=\int_{t_{1}}^{t_{2}} \tilde{f}_{0} d u=\int_{t_{1}}^{t_{2}} u^{0}(t) d t=x^{0}\left(t_{2}\right)
$$

Note that the set $\tilde{\tilde{Q}}(t, x)=\tilde{\tilde{f}}(t, x, \tilde{U}(t, x))$ of the new problem is the set of all $\tilde{z}=\left(z^{0}, z\right) \in E_{n+1}$ such that $z^{0}=u^{0}$, since $f_{0}=u^{0}, z=f(t, x, u), u^{0} \geqq f_{0}(t, x, u)$, $u \in U(t, x)$. Thus, the sets $\widetilde{U}, \tilde{\tilde{Q}}$ for this auxiliary problem are the sets $\tilde{U}, \tilde{\tilde{Q}}$ considered at the beginning of this proof.

We consider now the sequence of trajectories $\tilde{x}_{k}^{0}(t)=\left[x_{k}^{0}(t), x_{k}(t)\right], t_{1 k} \leqq t$ $\leqq t_{2 k}$, for the problem $J[\tilde{x}, \tilde{u}]$ corresponding to the control function $\tilde{u}(t)=\left[u_{k}^{0}(t)\right.$, $\left.u_{k}(t)\right]$ with $u_{k}^{0}(t)=f_{0}\left(t, x_{k}(t), u_{k}(t)\right), u_{k}(t) \in U\left(t, x_{k}(t)\right)$, and hence $\tilde{u}_{k}(t) \in \widetilde{U}\left(t, x_{k}(t)\right)$, $t_{1 k} \leqq t \leqq t_{2 k}, k=1,2, \cdots$. The sequence $\left[x_{k}(t)\right]$ converges in the metric $\rho$ toward the AC vector function $x(t)$, while $x_{k}^{0}(t) \rightarrow x^{0}(t)$ as $k \rightarrow+\infty$ for all $t \in\left(t_{1}, t_{2}\right)$, and $x^{0}(t)=Y(t)+Z(t)$, where $Y(t)$ is AC in $\left[t_{1}, t_{2}\right]$ and $Z^{\prime}(t)=0$ a.e. in $\left[t_{1}, t_{2}\right]$. 
By Closure Theorem II we conclude that $X(t)=[Y(t), x(t)]$ is a trajectory for the problem. In other words, there is a control function $\tilde{u}(t), t_{1} \leqq t \leqq t_{2}$, $\tilde{u}(t)=\left(u^{0}(t), u(t)\right)$, with

$$
d Y / d t=u^{0}(t) \geqq f_{0}(t, x(t), u(t)), \quad u(t) \in U(t, x(t)),
$$

a.e. in $\left[t_{1}, t_{2}\right]$, and

$$
d x / d t=f(t, x(t), u(t))
$$

$$
i \geqq Y\left(t_{2}\right)=J[\tilde{x}, \tilde{u}]=\int_{t_{1}}^{t_{2}} u^{0}(t) d t .
$$

First of all $[x(t), u(t)]$ is admissible for the original problem and hence belongs to $\Omega$, since by hypothesis $\Omega$ is complete. From this remark, and relations (4) and (5) we deduce

$$
i \leqq I[x, u]=\int_{t_{1}}^{t_{2}} f_{0}(t, x(t), u(t)) d t \leqq \int_{t_{1}}^{t_{2}} u^{0}(t) d t \leqq i,
$$

and hence all $\leqq$ signs can be replaced by $=$ signs, $u^{0}(t)=f_{0}(t, x(t), u(t))$ a.e. in $\left[t_{1}, t_{2}\right]$, and $I[x, u]=i$. This proves that $i$ is attained in $\Omega$. Existence Theorem $I$ is proved in the case $A$ is compact.

Let us assume now that $A$ is not compact but closed, that $A$ is contained in a slab $\left[t_{0} \leqq t \leqq T,-\infty<x^{i}<+\infty, i=1, \cdots, n, t_{0}, T\right.$ finite $]$, and that the additional hypotheses (a) and (b) hold. If $Z(t)$ denotes the scalar function $Z(t)$ $=|x(t)|^{2}+1$, then condition $x^{1} f_{1}+\cdots+x^{n} f_{n} \leqq C\left(|x|^{2}+1\right)$ implies $Z^{\prime} \leqq 2 C Z$, and hence, by integration from $t^{*}$ to $t$, also

$$
1 \leqq Z(t) \leqq Z\left(t^{*}\right) \exp 2 C\left|t-t^{*}\right|
$$

Since $\left[t^{*}, x\left(t^{*}\right)\right] \in P$ where $P$ is a compact subset of $A$, then there is a constant $N_{0}$ such that $|x| \leqq N_{0}$ for every $x \in P$, hence $1 \leqq Z\left(t^{*}\right) \leqq N_{0}^{2}+1$, and $1 \leqq Z(t)$ $\leqq\left(N_{0}^{2}+1\right) \exp 2 C\left(T-t_{0}\right)$. Thus, for $t_{0} \leqq t \leqq T, Z(t)$ remains bounded, and hence $|x(t)| \leqq D$ for some constant $D$. We can now restrict ourselves to the consideration of the compact part $A_{0}$ of all points $(t, x)$ of $A$ with $t_{0} \leqq t \leqq T$, $|x| \leqq D$.

Thus, Theorem $\mathrm{I}$ is proved for $A$ closed and contained in a slab as above and under the additional hypotheses (a), (b).

Let us assume that $A$ is not compact, nor contained in any slab as above but closed, and that hypotheses (a), (b), (c) hold. First, let us take an arbitrary element $\bar{x}(t), \bar{u}(t)$ of $\Omega$ and let $j=I[\bar{x}, \bar{u}]$. Then we consider an interval $(a, b)$ of the $t$-axis containing the entire projection $P_{0}$ of $P$ on the $t$-axis, as well as the interval $[-R, R]$. Now let $l=\mu^{-1}\left[|j|+1+(b-a) M_{0}\right]$, and let $\left[a^{\prime}, b^{\prime}\right]$ denote the interval $[a-l, b+l]$. Then for any admissible pair (if any) $x(t), u(t)$, $t_{1} \leqq t \leqq t_{2}$, of the class $\Omega$, whose interval $\left[t_{1}, t_{2}\right]$ is not contained in $\left[a^{\prime}, b^{\prime}\right]$, 
there is at least one point $t^{*} \in\left[t_{1}, t_{2}\right]$ with $\left(t^{*}, x^{*}(t)\right) \in P, a<t^{*}<b$, and a point $\bar{t} \in\left[t_{1}, t_{2}\right]$ outside $\left[a^{\prime}, b^{\prime}\right]$. Hence $\left[t_{1}, t_{2}\right]$ contains at least one subinterval, say $E$, outside $[a, b]$, of measure $\geqq l$. Then $I[x, u] \geqq l \mu-(b-a) M_{0}=|j|+1 \geqq i+1$. Obviously, we may disregard all pairs $x(t), u(t), t_{1} \leqq t \leqq t_{2}$, whose interval $\left[t_{1}, t_{2}\right]$ is not contained in $\left[a^{\prime}, b^{\prime}\right]$. In other words, we can limit ourselves to the closed part $A^{\prime}$ of all $(t, x) \in A$ with $a^{\prime} \leqq t \leqq b^{\prime}$. We are now in the situation above, and Theorem $\mathrm{I}$ is proved for any closed set $A$ under the additional hypotheses (a), (b), (c). Finally, we have to show that condition (a) can be replaced by condition $\left(\mathrm{a}^{\prime}\right)$. There are numbers $C, D>0$ such that $f_{0}(t, x, u) \geqq C|f(t, x, u)|$ for all $(t, x, u) \in M$ with $|x| \geqq D$. It is enough to prove Theorem I under the hypotheses that $A$ is closed and contained in a slab $t_{0} \leqq t \leqq T, t_{0}, T$ as above, and hypotheses $\left(a^{\prime}\right)$ and (b). First let us take $D$ so large that the projection $P^{*}$ of $P$ on the $x$-space is completely in the interior of the solid sphere $|x|<D$, and also so large that $D \geqq T-t_{0}$. Let $\bar{u}(t), \bar{x}(t)$ be any arbitrary admissible pair contained in $\Omega$, and let $j$ denote the corresponding value of the cost functional. Let $L=C^{-1}\left[D M_{0}+|j|+1\right]$, and let us take $D_{0}=D+L$. If any admissible pair $u(t), x(t), t_{1} \leqq t \leqq t_{2}$, of $\Omega$ possesses a point $\left(t_{0}, x\left(t_{0}\right)\right)$ with $\left|x\left(t_{0}\right)\right| \geqq D_{0}$, then $x(t)$ possesses also a point $\left(t^{*}, x\left(t^{*}\right)\right) \in P$, with $\left|x\left(t^{*}\right)\right| \leqq D$. Thus, there is at least a subarc $\Gamma: x=x(t), t^{\prime} \leqq t \leqq t^{\prime \prime}$ of $x(t)$ along which $|x(t)| \geqq D$ and $|x(t)|$ passes from the value $D$ to the value $D_{0}=D+L$. Such an arc $\Gamma$ has a length $\geqq L$. If $E=\left[t_{1}, t_{2}\right]-\left[t^{\prime}, t^{\prime \prime}\right]$, then

$$
\begin{aligned}
I[x, u] & =\int_{t_{1}}^{t_{2}} f_{0} d t=\left(\int_{E}+\int_{t^{\prime}}^{t^{\prime \prime}}\right) f_{0} d t \geqq-D M_{0}+\int_{t^{\prime}}^{t^{\prime \prime}} C|f| d t \\
& =-D M_{0}+C \int_{t^{\prime}}^{t^{\prime \prime}}|d x / d t| d t=-D M_{0}+C L=|j|+1 \geqq i+1 .
\end{aligned}
$$

As before we can restrict ourselves to the compact part $A_{0}$ of all points $(t, x)$ of $A$ with $t_{0} \leqq t \leqq T,|x| \leqq D$. The case where $A$ is closed, $A$ is not contained in any slab as above, but conditions ( $\left.\mathrm{a}^{\prime}\right)$, (b), (c) hold, can be treated as before. The case where $A$ is not compact and the condition $(\gamma)$ holds, also can be treated as before. Theorem $\mathrm{I}$ is thereby completely proved.

REMARK 1. If the set

$$
\begin{aligned}
\widetilde{Q}(t, x) & =\tilde{f}[t, x, U(t, x)]=\left[\tilde{z}=\left(z^{0}, z\right) \mid \tilde{z}=\tilde{f}(t, x, u), u \in U(t, x)\right] \\
& =\left[\tilde{z}=\left(z^{0}, z\right) \mid z^{0}=f_{0}(t, x, u), z=f(t, x, u), u \in U(t, x)\right] \subset E_{n+1}
\end{aligned}
$$

is convex, then certainly the set $\tilde{\tilde{Q}}(t, x)$ of Theorem $I$ is convex also. On the other hand, trivial examples show that $\tilde{\widetilde{Q}}(t, x)$ may be convex, when $Q(t, x)$ is not. This is actually the usual case in free problems of the calculus of variations (see Remark 3 below). Thus, the requirement in Theorem I that $\tilde{\tilde{Q}}(t, x)$ be convex for every $(t, x)$ is a wide generalization of the analogous hypothesis concerning 
$\widetilde{Q}(t, x)$ which is familiar in Pontryagin's problems. For these problems, Filippov's existence theorem is a particular case of Theorem I.

The theorem of A. F. Filippov [2]. As in Theorem I, if $A=E_{1} \times E_{n}$, if $f(t, x, u)=\left(f_{0}, f\right)=\left(f_{0}, f_{1}, \cdots, f_{n}\right)$ is continuous in $M$, if $U(t, x)$ is compact for every $(t, x)$ in $A$, if $U(t, x)$ is an upper semicontinuous function of $(t, x)$ in $A$, if $\tilde{Q}(t, x)=\tilde{f}(t, x, U(t, x))$ is a convex subset of $E_{n+1}$ for every $(t, x)$ in $A$, if conditions (a) and (c) are satisfied, and the class $\Omega$ of all admissible pairs for which $x\left(t_{1}\right)=x_{1}, x\left(t_{2}\right)=x_{2}, t_{1}, x_{1}, x_{2}$ fixed, $t_{2}$ undetermined, is not empty, then $I[x, u]$ has absolute minimum in $\Omega$.

This statement is a corollary of Theorem I. Indeed, under hypothesis (c) we can restrict $A$ to the closed part $A_{0}$ of all $(t, x) \in A$ with $a^{\prime} \leqq t \leqq b^{\prime}$, and $|x| \leqq N$ for some large $N$. If $M_{0}$ is the part of all $(t, x, u)$ of $M$ with $(t, x) \in A_{0}$, then the hypothesis that $U(t, x)$ is compact and an upper semicontinuous function of $(t, x)$ in $A_{0}$ certainly implies that $U(t, x)$ satisfies condition $(U)$ in $A_{0}$ and that $M_{0}$ is compact ( $\S 4$, (vi) and (vii)). Also, since $\widetilde{Q}(t, x)$ is convex for every $(t, x)$ by hypothesis, we deduce that $Q(t, x)$ is an uppersemicontinuous function of $(t, x)$ and satisfies property $(Q)(\S 4$, (xii) and (xiii)). Also, $\tilde{\widetilde{Q}}(t, x)$ is closed, convex, and satisfies condition $(Q)$ by force of Lemma (xvi) of $\S 4$. Finally, since $M_{0}$ is compact, the growth condition $f_{0} \geqq \Phi$ and the remaining condition $|f| \leqq C+D|u|$ are trivially satisfied. Thus, all conditions of Theorem I are satisfied, and Filippov's theorem is proved to be a particular case of Theorem I.

REMARK 2. The analogous existence theorems of E. Roxin [8] and of L. Markus and E. B. Lee [14] are also essentially contained in Theorem I. For a detail on Roxin's statement see Remark 4 below.

REMARK 3. For free problems of the calculus of variations ( $\$ 11$ below) we have $m=n, U=E_{n}, f=u$, hence

$$
\begin{aligned}
& \tilde{Q}(t, x)=\tilde{f}[t, x, U(t, x)]=\left[\tilde{z}=\left(z^{0}, u\right) \mid z^{0}=f_{0}(t, x, u), u \in E_{n}\right] \subset E_{n+1}, \\
& \tilde{Q}(t, x)=\left[\tilde{z}=\left(t^{0}, u\right) \mid z^{0} \geqq f_{0}(t, x, u), u \in E_{n}\right] \subset E_{n+1} .
\end{aligned}
$$

The set $\widetilde{Q}$ is convex if and only if $f_{0}$ is linear in $u$, while $\widetilde{Q}$ is convex if and only if $f_{0}$ is convex in $u$. Thus condition $\widetilde{\tilde{Q}}$ convex on Theorem I reduces to the requirement $f_{0}$ convex in $u$ which is familiar for free problems in the calculus of variations. We shall prove in $\$ 11$ that the Nagumo-Tonelli existence theorem for free problem is also a particular case of Theorem $I$.

REMARK 4. The condition $f_{0} \geqq \Phi(|u|)$ with $\Phi(z) / z \rightarrow+\infty$ of the theorems above is said to be a growth condition on $f_{0}$. As it is well known such a condition (for $f_{0}$ convex in $u, A$ compact, and $U=E_{m}$ ) is equivalent to the condition that, for every $(t, x) \in A$, we have $f_{0}(t, x, u) /|u| \rightarrow+\infty$ as $|u| \rightarrow+\infty$ (L. Tonelli, [9a]). On the other hand, it is known already for free problems, that if such a 
condition is not satisfied at the points $(t, x) \in A$ of even only one hyperplane $t=\bar{t}$, then the absolute minimum need not exist (see [9b], and $\$ 10$ below, example 2). For free problems, other additional conditions have been devised in such cases [9a].

Condition (a) $x^{1} f_{1}+\cdots+x^{n} f_{n} \leqq C\left(|x|^{2}+1\right)$ of Theorem I can be replaced by $x^{1} f_{1}+\cdots+x^{n} f_{n} \leqq \phi(t)\left(|x|^{2}+1\right)$, where $\phi(t) \geqq 0$ is a fixed function of $t$ which is $L$-integrable in any finite interval. The remark was made by E. O. Roxin [8] in connection with Pontryagin's problems.

Condition (a) could also be replaced by the following general assumption from differential equations theory: there exists a (Lyapunov-like) positive, continuously differentiable function $V(x, t)$ and a positive constant $c$ such that

$$
\left|\operatorname{grad}_{x} V(x, t) \cdot f(t, x, u)+\partial V / \partial t\right| \leqq c V(x, t)
$$

for all $(t, x, u) \in M$, and the set

$$
\{x \mid V(x, t) \leqq \alpha,(t, x) \in A\}
$$

is compact for every $\alpha$.

REMARK 5. For problems of optimal control where $U(t, x)$ is always compact we have given in [1bc] an existence theorem, say $I^{*}$, similar to the Filippov's theorem above, where the condition " $\tilde{Q}$ convex" is replaced by the following requirement: $Q(t, x)$ is a convex subset of $E_{n}, f_{0}(t, x, u), u \in U(t, x)$, is convex in $u$, and "the curvature of $f$ is always small with respect to the convexity of $f_{0}$ ", (see [1b], or [1c] for a precise statement). Whenever this requirement implies the convexity of the set $\tilde{\tilde{Q}}$, then the theorem given in [1bc] becomes a corollary of Theorem I above. Also it should be pointed out that, whenever the relation $z=f(t, x, u)$ between $Q(t, x)$ and $U(t, x)$ can be inverted and $u=f^{-1}(t, x, z)$ is a continuous function of $z$ in $Q(t, x)$, then the set $\tilde{\tilde{Q}}(t, x)$ can be represented by

where

$$
\tilde{\tilde{Q}}(t, x)=\left[\tilde{z}=\left(z^{0}, z\right) \mid z^{0} \geqq F(t, x, z), z \in Q(t, x)\right],
$$

$$
F(t, x, z)=f_{0}\left(t, x, f^{-1}(t, x, z)\right),
$$

and thus the requirement of the convexity of the set $\tilde{\tilde{Q}}$ reduces to the requirement of the convexity of the function $F(t, x, z)$ in $z$. Then the further requirement that $\widetilde{\widetilde{Q}}(t, x)$ satisfies property $(Q)$ is certainly satisfied if, besides, $F$ is quasi normally convex as proved in $[\$ 4,(x v i i)]$. We discussed in [1c] a case where the requirement of Theorem $I^{*}$ implies the convexity of $F$ in $u$, and correspondingly $I^{*}$ becomes a corollary of $\mathrm{I}$. The simpler requirement: $Q(t, x)$ a convex subset of $E_{n}$ and $f_{0}(t, x, u)$ convex in $u$, does not suffice for existence, as we prove in the following number.

8. Example of a problem with no absolute minimum. The condition " $\tilde{\widetilde{Q}}(t, x)$ convex for every $(t, x) \in A$ " of Theorem I cannot be replaced by the simpler 
condition " $Q(t, x)$ convex for every $(t, x) \in A$ and $f_{0}(t, x, u)$ convex in $u$," not even when $A$ and all sets $U(t, x)$ are compact (that is, for Pontryagin problems). This is shown by the following example.

Let us consider the differential system:

$$
\begin{aligned}
& x^{\prime}=u(1-v)+\left[2-2^{-1}(u-1)^{2}\right] v, \\
& y^{\prime}=\left[2-2^{-1}(u-1)^{2}\right](1-v)+u v,
\end{aligned}
$$

with $t_{1}=0$, initial point $(0,0)$, fixed target $(0,1)$, and fixed control space $U=[-1 \leqq u \leqq 1,0 \leqq v \leqq 1]$. If

$$
\begin{aligned}
& z_{1}=f_{1}=u(1-v)+\left[2-2^{-1}(u-1)^{2}\right] v, \\
& z_{2}=f_{2}=\left[2-2^{-1}(u-1)^{2}\right](1-v)+u v,
\end{aligned}
$$

we see that the segment $[v=1,-1 \leqq u \leqq 1]$ is mapped by $f=\left(f_{1}, f_{2}\right)$ onto the arc of parabola $A B C=\left[z_{1}=2-2^{-1}(u-1)^{2}, z_{2}=u,-1 \leqq u \leqq 1\right]$, whose points $A=(0,-1), B=(3 / 2,0), C=(2,1)$ correspond to $u=-1,0,1$ respectively. The segment $[v=0,-1 \leqq u \leqq 1]$ is mapped by $f$ onto the arc $D E F=\left[z_{1}=u, z_{2}=2-2^{-1}(u-1)^{2},-1 \leqq u \leqq 1\right]$, whose points $D=(-1,0)$, $E=(0,3 / 2), F=(1,2)$ correspond to $u=-1,0,1$ respectively. Each segment $[u=c, 0 \leqq v \leqq 1]$ is mapped by $f$ onto the segment joining the points corresponding to $(c, 1)$ and $(c, 0)$ on the two parabolas. Thus, the image $Q=f(U)$ of $U$ is the convex body $Q=(A B C F E D)$ of the $z_{1} z_{2}$-plane. Let us consider the cost functional

$$
I=\int_{t_{1}}^{t_{2}}\left[x^{2}+(y-t)^{2}+v^{2}\right] d t .
$$

For $k=1,2, \cdots$, let $u_{k}(t), v_{k}(t), 0 \leqq t \leqq 1$, be defined by taking $u_{k}(t)=-1$, $v_{k}(t)=0$, or $u_{k}(t)=+1, v_{k}(t)=0$, according as $t$ belongs to the intervals $k^{-1}(i-1)<t<k^{-1}(i-1)+(2 k)^{-1}$, or $k^{-1}(i-1)+(2 k)^{-1}<t<k^{-1} i$, $i=1,2, \cdots, k$. Then the functions $x_{k}(t), y_{k}(t), 0 \leqq t \leqq 1$, satisfy the differential equations $d x_{k} / d t=+1, d y_{k} / d t=2$, or $d x_{k} / d t=-1, d y_{k} / d t=0$, according as $t$ belongs to one or the other of the two sets of intervals above. Then $x_{k}(t) \rightarrow x_{0}(t)=0$, $y_{k}(t) \rightarrow y_{0}(t)=t$ uniformly in $0 \leqq t \leqq 1$ as $k \rightarrow \infty$. If $C_{k}, C_{0}$ denote these trajectories we say that $C_{k} \rightarrow C_{0}$.

The question as to whether $C_{0}$ is actually a trajectory, that is, whether there are admissible control functions $u_{0}(t), v_{0}(t), 0 \leqq t \leqq 1$, whose corresponding trajectory is $C_{0}$ can be answered in the affirmative because of the convexity of $Q$. Actually, the point $\left(\alpha_{0}, \beta_{0}\right) \in U, \alpha_{0}=2-5^{1 / 2}=-0.23607, \beta_{0}=(11)^{-1}\left(4-5^{1 / 2}\right)$ $=0.16036$, is mapped by $f$ into $\left(z_{1}=0, z_{2}=1\right)$, and thus $z_{0}(t)=\alpha_{0}, v_{0}(t)$ $=\beta_{0}, 0 \leqq t \leqq 1$, generate $C_{0}$. Now we have $x_{k}(t) \rightarrow 0, y_{k}(t) \rightarrow t$, uniformly in 
$[0,1]$ as $k \rightarrow \infty$, and $v_{k}(t)=0$, hence $I\left[C_{k}\right] \rightarrow 0$ as $k \rightarrow \infty$. On the other hand $I\left[C_{0}\right]=\int_{0}^{1}\left(0^{2}+0^{2}+\beta_{0}^{2}\right) d t=\beta_{0}^{2}>0$.

Let us prove that $I$ has no absolute minimum in the class $\Omega$ of all trajectories satisfying the differential equations, boundary conditions, and constraints above. Indeed, $I\left[C_{k}\right] \rightarrow 0$ shows that the infimum of $I[C]$ in $\Omega$ is zero, but this value cannot be attained in $\Omega$ by $I$. Indeed, $I[C]=0$ implies $x=0, y=t, v=0$, and the first two relations alone imply $u=\alpha_{0}, v=\beta_{0} \neq 0$ a.e. in $[0,1]$, a contradiction. Thus $I$ cannot attain the value zero in $\Omega$.

In this example $Q$ is a convex set, $f_{0}$ is convex in $(u, v)$, and even satisfies trivially the growth condition $f_{0} \geqq \Phi$, since here $U$ is a bounded set. Now let us prove that $\tilde{\widetilde{Q}}$ is not convex. It is enough to verify this for $t=0, x=0, y=0$. Then $\widetilde{\tilde{Q}}$ is simply the set of all $z=\left(z_{0}, z_{1}, z_{2}\right)$ with $\left(z_{1}, z_{2}\right) \in Q$ satisfying the relation $z_{0} \geqq f_{0}=v^{2}$, when $z_{1}, z_{2}, u, v$ are related by $z_{1}=f_{1}, z_{2}=f_{2},(u, v) \in U$. Now the segment $\tau=[v=0,-1 \leqq u \leqq 1]$ is mapped by $f$ onto the $\operatorname{arc} \Gamma=(D E F) \subset Q$, and we have $f_{0}>0$ in $Q-\Gamma, f_{0}=0$ in $\Gamma$, and hence $\tilde{\tilde{Q}}$ convex would imply that $\Gamma$ is a segment, and this is not the case. This proves that $\widetilde{\widetilde{Q}}$ is not a convex set.

\section{Another existence theorem for Lagrange problems with unilateral constraints.}

EXISTENCE THEOREM II. Let $A$ be a compact subset of the tx-space $E_{1} \times E_{n}$, and, for every $(t, x) \in A$, let $U(t, x)$ be a closed subset of the u-space $E_{m}$. Let $f(t, x, u)=\left(f_{0}, f_{1}, \cdots, f_{n}\right)=\left(f_{0}, f\right)$ be a continuous vector function on the set $M$ of all $(t, x, u)$ with $(t, x) \in A, u \in U(t, x)$. Assume that, for every $(t, x) \in A$, the set

$$
\tilde{\tilde{Q}}(t, x)=\left[\tilde{z}=\left(z^{0}, z\right) \in E_{n+1} \mid z^{0} \geqq f_{0}(t, x, u), z=f(t, x, u), u \in U(t, x)\right]
$$

is convex, and that $U(t, x)$ satisfies property $(U)$ and $\widetilde{\tilde{Q}}(t, x)$ satisfies property $(Q)$ in $A$. Let $\phi(t)$ be a given function which is L-integrable in any finite interval such that $f_{0}(t, x, u) \geqq \phi(t)$ for all $(t, x, u) \in M$. Let $\Omega$ be a nonempty complete class of admissible pairs $x(t), u(t)$ such that

$$
\left.\int_{t_{1}}^{t_{2}}\left|d x^{i}\right| d t\right|^{p} d t \leqq N_{i}, \quad i=1, \cdots, n,
$$

for some constants $N_{i} \geqq 0, p>1$. Then the cost functional $I[x, u]$ has an absolute minimum in $\Omega$.

If $A$ is not compact, but closed and contained in a slab $\left[t_{0} \leqq t \leqq T,-\infty\right.$ $<x^{i}<+\infty, i=1, \cdots, n, t_{0}, T$ finite], then Theorem II still holds under the additional hypothesis (b) after Theorem $\mathrm{I}$. If $A$ is not compact, nor contained in a slab as above, but $A$ is closed, then Theorem II still holds under the additional hypotheses (b) and ( $\left.\mathrm{c}^{*}\right): f_{0}(t, x, u) \geqq \phi(t)$ for all $(t, x, u) \in M$ where $\phi(t)$ is a given function which is $L$-integrable in any finite interval and 
$\int_{0}^{+\infty} \phi(t) d t=+\infty, \int_{-\infty}^{0} \phi(t) d t=+\infty$. Finally, if for some $i=1, \cdots, n$, and any $N>0$, there is some $N_{i}>0$ such that $(x, u) \in \Omega, I[x, u] \leqq N$ implies $\int_{t_{1}}^{t_{2}}\left|d x^{i} / d t\right|^{p} d t \leqq N_{i}$, then the corresponding requirement (24) can be disregarded.

Proof of Existence Theorem II. We suppose $A$ compact, hence necessarily contained in a slab $\left[t_{0} \leqq t \leqq T, t_{0}, T\right.$ finite, $\left.-\infty<x^{i}<+\infty, i=1, \cdots, n\right]$, and then $I[x, u]=\int_{t_{1}}^{t_{2}} f_{0} d t \geqq-\int_{t_{0}}^{T}|\phi(t)| d t$. This proves that the infimum $i$ of $I[x, u]$ in $\Omega$ is necessarily finite. Let $u_{k}(t), x_{k}(t), t_{1 k} \leqq t \leqq t_{2 k}, k=1,2, \cdots$, be a sequence of admissible pairs all in $\Omega$ with $I\left[x_{k}, u_{k}\right] \rightarrow i$. We may assume

$$
i \leqq I\left[x_{k}, u_{k}\right]=\int_{t_{1 k}}^{t_{2 k}} f_{0}\left(t, x_{k}(t), u_{k}(t)\right) d t \leqq i+1 / k \leqq i+1 .
$$

Then

$$
\int_{t_{1 k}}^{t_{2 k}}\left|d x_{k}^{i} / d t\right|^{p} d t \leqq N_{i}, \quad i=1, \cdots, n, \quad k=1,2, \cdots
$$

By the weak compactness of $L_{p}$ we conclude that there is some subsequence and some AC vector function $x(t)=\left(x^{1}, \cdots, x^{n}\right), t_{1} \leqq t \leqq t_{2}$, such that $t_{1 k} \rightarrow t_{1}, t_{2 k} \rightarrow t_{2}$, $d x_{k}^{i} / d t \rightarrow d x^{i} / d t$ weakly in $L_{p}, x_{k}(t) \rightarrow x(t)$ in the $\rho$-metric. The proof is now exactly the same as for Existence Theorem I.

If $A$ is not compact, but closed and contained in a slab as above, and condition (b) holds, then for every admissible pair $u(t), x(t)$ of $\Omega$ we have

$$
\begin{aligned}
\left|x(t)-x\left(t^{*}\right)\right| & =\left|\int_{t^{*}}^{t}(d x / d t) d t\right| \leqq\left|\int_{t^{*}}^{t} d t\right|^{1 / q}\left|\int_{t^{*}}^{t}\right| d x /\left.\left.d t\right|^{p} d t\right|^{1 / p} \\
& \leqq\left|t-t^{*}\right|^{1 / q}\left(N_{1}+\cdots+N_{n}\right),
\end{aligned}
$$

where $\left(t^{*}, x\left(t^{*}\right)\right)$ belongs to a fixed compact subset $P$ of $A$. Then $\left|x\left(t^{*}\right)\right| \leqq N^{\prime}$, $\left|t-t^{*}\right| \leqq T-t_{0}$, and $|x(t)| \leqq N^{\prime \prime}$ for some constants $N^{\prime}, N^{\prime \prime}>0$. Thus, we can limit ourselves to the compact part $A_{0}$ of all points $(t, x)$ of $A$ with $t_{0} \leqq t \leqq T$, $|x| \leqq N^{\prime \prime}$. If $A$ is not compact, nor contained in a slab as above, but $A$ is closed and conditions (b), (c) hold, then we can use the same argument as for Existence Theorem I.

Finally, we see that assumption (24) has been used only in (26) for a minimizing sequence $u_{k}, x_{k}$. Since for a minimizing sequence we see already in (25) that $I\left[u_{k}, x_{k}\right] \leqq i+1$, it is obvious that any relation (24) which is a consequence of a relation of the form $I \leqq N$ need not be required among the assumptions of Theorem II. Theorem II is thereby proved.

10. Examples.

1. Let us consider the (free) problem

$$
I[x]=\int_{t_{1}}^{t_{2}}\left(1+\left|x^{\prime}\right|^{2}\right) d t=\text { minimum }
$$


with $x=\left(x^{1}, \cdots, x^{n}\right)$, in the class $\Omega$ of all absolutely continuous functions $x(t)=\left(x^{1}, \cdots, x^{n}\right), 0 \leqq t \leqq t_{2}$, whose graphs $(t, x(t))$ join the point $\left(t_{1}=0, x\left(t_{1}\right)\right.$ $=(0, \cdots, 0))$ to a nonempty closed set $B$ of the half-space $t_{2} \geqq 0, x \in E_{n}$. This problem can be written as a Lagrange problem:

$$
\begin{aligned}
& J[x, u]=\int_{t_{1}}^{t_{2}}\left(1+|u(t)|^{2}\right) d t=\text { minimum, } \\
& d x / d t=u^{t}, \quad i=1, \cdots, n,
\end{aligned}
$$

where $x(t)=\left(x^{1}, \cdots, x^{n}\right), \quad u(t)=\left(u^{1}, \cdots, u^{n}\right), \quad m=n, \quad f_{0}=1+|u|^{2}, \quad f_{i}=u^{i}$, $i=1, \cdots, n$, and the control space $U(t, x)$ is fixed and coincides with the whole space $E_{n}$. Here $\tilde{\tilde{Q}}(t, x)=\left[\left.(z, u)|z \geqq 1+| u\right|^{2}, u \in E_{n}\right]$ is a fixed and convex subset of $E_{n+1}$. The conditions of Theorem I are satisfied with $\Phi(|u|)=\left|u^{2}\right|$, or $\Phi(z)=z^{2}, 0 \leqq z \leqq+\infty, A$ is the half-space $A=\left[(t, x) \mid t \geqq 0, x \in E_{n}\right] \subset E_{n+1}$. Thus the problem above has an optimal solution.

2. The free problem

$$
I[x]=\int_{0}^{1} t x^{\prime 2} d t=\text { minimum }, \quad x(0)=1, x(1)=0,
$$

is known to have no optimal solution [9b]. The same problem can be written as a Lagrange problem with $m=n=1$ in the form

$$
\begin{gathered}
J_{1}[x, u]=\int_{0}^{1} t u^{2} d t=\text { minimum, } \quad x(0)=1, \quad x(1)=0 . \\
d x / d t=u, \quad u \in E_{1},
\end{gathered}
$$

as well as in the form

$$
\begin{gathered}
J_{2}[x, u]=\int_{0}^{1} t^{3} u^{2} d t=\text { minimum }, \quad x(0)=1, x(1)=0, \\
d x / d t=t u, \quad u \in E_{1} .
\end{gathered}
$$

The relative sets $\tilde{\tilde{Q}}(t, x)$ are here subsets of the $z^{0} z$-plane $E_{2}$. For the problem $J_{1}$ the sets $\tilde{\tilde{Q}}$ satisfy condition $(Q)$, but $f_{0}=t u^{2}$ does not satisfy the growth condition of Theorem I. For the problem $J_{2}$ the sets $\tilde{\tilde{Q}}$ do not satisfy condition $(Q)$. (We shall take into consideration the same sets under examples 4 and 5 of $\S 12$ below.)

The same free problem with an additional constraint

$$
\int_{0}^{1} x^{\prime 2} d t \leqq N_{0}
$$

where $N_{0} \geqq 1$ is any constant, has an optimal solution by force of Theorem II and subsequent remark. The optimal solution will depend on $N_{0}$. Note that 
$N_{0} \geqq 1$ assures that the class $\Omega$ relative to the problem is not empty. Indeed for $x(t)=1-t$, we have $\int_{0}^{1} x^{\prime 2} d t=1$.

11. The free problems. If we assume $m=n, f_{i}=u_{i}, i=1, \ldots, n, U(t, x)=E_{m}$, then the differential system reduces to $d x / d t=u$, and the cost functional to

$$
I[x, u]=\int_{t_{1}}^{t_{2}} f_{0}(t, x(t), u(t)) d t=\int_{t_{1}}^{t_{2}} f_{0}\left(t, x(t), x^{\prime}(t)\right) d t .
$$

Then the problem under consideration (no. 6) reduces to a free problem (no differential system) where the integral is written in the form

$$
I[x]=\int_{t_{1}}^{t_{2}} f_{0}\left(t, x(t), x^{\prime}(t)\right) d t,
$$

and the only constraint is now $(t, x(t)) \in A$ for all $t_{1} \leqq t \leqq t_{2}$. Again, complete classes $\Omega$ of vector functions $x(t)$ can be defined by means of boundary conditions of the type $\left(t_{1}, x\left(t_{1}\right), t_{2}, x\left(t_{2}\right)\right) \in B$, where $B$ is a closed subset of $E_{2 n+2}$ as in $\S 6$.

The Nagumo-Tonelli Theorem [9 ac, 5]. If $A$ is a compact subset of the tx-space $E_{1} \times E_{n}$, if $f_{0}(t, x, u)$ is a continuous function on the set $M=A \times E_{n}$, if for every $(t, x) \in A, f_{0}(t, x, u)$ is convex as a function of $u$ in $E_{n}$, if there is a continuous scalar function $\Phi(\zeta), 0 \leqq \zeta<+\infty$, with $\Phi(\zeta) / \zeta+\rightarrow \infty$ as $\zeta+\rightarrow \infty$, such that $f_{0}(t, x, u) \geqq \Phi(|u|)$ for all $(t, x, u) \in M$, then the cost functional (1) has an absolute minimum in any nonempty complete class $\Omega$ of absolutely continuous vector functions $x(t), t_{1} \leqq t \leqq t_{2}$, for which $f_{0}\left(t, x(t), x^{\prime}(t)\right)$ is L-integrable in $\left[t_{1}, t_{2}\right]$.

If $A$ is not compact, but closed and contained in a slab $\left[t_{0} \leqq t \leqq T, x \in E_{n}\right]$, $t_{0}, T$ finite, then the statement still holds under the additional hypotheses $\left(\tau_{1}\right) f_{0} \geqq C|u|$ for all $(t, x, u) \in M$ with $|x| \geqq D$ and convenient constants $C>0, D \geqq 0 ;\left(\tau_{2}\right)$ every trajectory $x(t)$ of $\Omega$ possesses at least one point $\left(t^{*}, x\left(t^{*}\right)\right)$ on a given compact subset $P$ of $A$. If $A$ is not compact, nor contained in a slab as above, but $A$ is closed, then the statement still holds under the additional hypotheses $\left(\tau_{1}\right),\left(\tau_{2}\right)$, and $\left(\tau_{3}\right) f_{0}(t, x, u) \geqq \mu>0$ for all $(t, x, u) \in M$ with $|t| \geqq R$, and convenient constants $\mu>0$ and $R \geqq 0$.

Proof. First assume $A$ to be compact. Then the set $\tilde{\tilde{Q}}(t, x)$ reduces here to the set of all $\tilde{z}=\left(z^{0}, z\right) \in E_{n+1}$ with $z^{0} \geqq f_{0}(t, x, z), z \in E_{n}$, where $f_{0}$ is convex in $z$, and satisfies the growth condition $f_{0} \geqq \Phi(|u|)$ with $\Phi(\zeta) / \zeta \rightarrow+\infty$ as $\zeta \rightarrow+\infty$. By the remark after Lemma (xv) of $\S 4, f_{0}$ is normally convex in $u$, hence quasi normally convex, and, by Lemma (xvi), part $(\beta)$, of $\S 4, \tilde{\widetilde{Q}}$ satisfies condition $(Q)$ in $A$. Thus, all hypotheses of Theorem I of $\S 7$ are satisfied. If $A$ is closed but contained in a slab as above then the condition (a) of Theorem I reduces to $u \cdot x \leqq C\left(|x|^{2}+1\right)$ which cannot be satisfied since we have no bound on $u$. On the other hand, the condition ( $\left.\mathrm{a}^{\prime}\right) f_{0} \geqq C|f|$ for some $C>0$ reduces here to requirement $\left(\tau_{1}\right)$ and condition (b) to requirement $\left(\tau_{2}\right)$. Finally, if $A$ is 
not compact, nor contained in a slab as above, but $A$ is closed, then requirement (c) of Theorem I reduces to requirement $\left(\tau_{3}\right)$. All conditions of Theorem I are satisfied, and the cost functional (1) has an absolute minimum in $\Omega$.

12. Lagrange problems with $f$ linear in $u$. We shall consider now the case where all functions $f_{i}(t, x, u), i=1, \cdots, n$, are linear in $u$, and the control space $U(t, x)$ is fixed and coincides with the total space $E_{m}$. Precisely, we shall consider the Lagrange problem

$$
\begin{aligned}
& I[x, u]=\int_{t_{1}}^{t_{2}}\left[g(t, x) \phi(u)+g_{0}(t, x)\right] d t=\text { minimum }, \\
& d x^{i} / d t=\sum_{j=1}^{m} g_{i j}(t, x) u^{j}+g_{i}(t, x), \quad i=1, \cdots, n,
\end{aligned}
$$

where $x=\left(x^{1}, \cdots, x^{n}\right) \in E_{m}$, and $\phi(u), u \in U=E_{m}$, is a convex function of $u$ satisfying a growth condition as in Nagumo-Tonelli Theorem. If $H(t, x)$ denotes the $n \times m$ matrix $\left(g_{i j}(t, x)\right)$, and $h(t, x)$ the $n$-vector $\left(g_{i}(t, x)\right)$, then the differential system (2) takes the form $d x / d t=H(t, x) u+h(t, x)$.

The sets $Q(t, x), \tilde{\widetilde{Q}}(t, x)$ relative to the problem above are

$$
Q(t, x)=\left[z \mid z=H(t, x) u+h(t, x), u \in E_{m}\right] \subset E_{n},
$$

$\tilde{\tilde{Q}}(t, x)=\left[\tilde{z}=\left(z^{0}, z\right) \mid z^{0} \geqq g(t, x) \phi(u)+g_{0}(t, x), z=H(t, x) u+h(t, x), u \in E_{n}\right] \subset E_{n+1}$.

Obviously, $Q(t, x)$ is a $r$-dimensional linear manifold in $E_{n}$ where $r$ is the rank of $H(t, x)$. We shall need a few lemmas concerning the sets $Q(t, x)$.

(i) If $g$ is nonnegative, and $\phi$ is nonnegative and convex, then both sets $Q(t, x), \widetilde{Q}(t, x)$ defined in (3) are convex for every $(t, x) \in A$.

Proof. We give the proof for $\tilde{\tilde{Q}}(t, x)$. Let $\tilde{\xi}=\left(\xi^{0}, \xi\right), \tilde{\eta}=\left(\eta^{0}, \eta\right)$ be any two points of $\tilde{\tilde{Q}}(t, x)$, let $0 \leqq \alpha \leqq 1$, and $\tilde{z}=\left(z^{0}, z\right)=\alpha \tilde{\xi}+(1-\alpha) \tilde{\eta}$. Then for some vectors $u, v \in E_{m}$ we have

$$
\begin{aligned}
& \xi^{0} \geqq g \phi(u)+g_{0}, \quad \xi=H u+h, \\
& \eta^{0} \geqq g \phi(v)+g_{0}, \quad \eta=H v+h, \\
& \tilde{z}=\alpha \tilde{\xi}+(1-\alpha) \tilde{\eta}, \quad z^{0}=\alpha \xi^{0}+(1-\alpha) \eta^{0}, \quad z=\alpha \xi+(1-\alpha) \eta .
\end{aligned}
$$

If $w \in E_{m}$ denotes the vector $w=\alpha u+(1-\alpha) v$, we have

$$
\begin{aligned}
z & =\alpha \xi+(1-\alpha) \eta=\alpha(H u+h)+(1-\alpha)(H v+h) \\
& =H(\alpha u+(1-\alpha) v)+h=H w+h, \\
z^{0} & =\alpha \xi^{0}+(1-\alpha) \eta^{0} \geqq \alpha\left(g \phi(u)+g_{0}\right)+(1-\alpha)\left(g \phi(v)+g_{0}\right) \\
& =g(\alpha \phi(u)+(1-\alpha) \phi(v))+g_{0} \\
& \geqq g \phi(\alpha u+(1-\alpha) v)+g_{0}=g \phi(w)+g_{0} .
\end{aligned}
$$


Thus, $\tilde{z}=\left(z^{0}, z\right) \in \tilde{\tilde{Q}}(t, x)$ and $\tilde{\tilde{Q}}(t, x)$ is convex.

(ii) If all functions $\phi, g, g_{0}, g_{i j}, g_{i}$ are continuous, if $\phi(u)$ is nonnegative and convex, and there is a function $\Phi(\zeta), 0 \leqq \zeta<+\infty$, such that $\Phi(\zeta) \rightarrow+\infty$ as $\zeta \rightarrow+\infty$, and $\phi(u) \geqq \Phi(|u|)$ for all $u \in E_{m}$, if there is a neighborhood $N_{\delta}(\bar{t}, \bar{x})$ of $(\bar{t}, \bar{x})$ where $g \geqq \mu$ for some constant $\mu>0$, then the set $\tilde{\tilde{Q}}(t, x)$ defined in (3) satisfies property $(Q)$ at $(\bar{t}, \bar{x})$.

Proof. We have to prove that $\underset{\tilde{Q}}{\tilde{Q}}(\bar{t}, \bar{x})=\bigcap_{\delta} \mathrm{cl} \operatorname{co} \tilde{\tilde{Q}}(\bar{t}, \bar{x}, \delta)$. It is enough to prove that $\bigcap_{\delta} \operatorname{cl} \operatorname{co} \tilde{\tilde{Q}}(\bar{t}, \bar{x}, \delta) \subset \tilde{\tilde{Q}}(\tilde{t}, \bar{x})$ since the opposite inclusion is trivial. Let us assume that a given point $\tilde{z}=\left(\bar{z}^{0}, \bar{z}\right) \in \bigcap_{\delta} \operatorname{cl} \operatorname{co} \widetilde{Q}(\bar{t}, \bar{x}, \delta)$ and let us prove that $\tilde{z}=\left(\bar{z}^{0}, \bar{z}\right) \in \tilde{\tilde{Q}}(\bar{i}, \bar{x})$. For every $\delta>0$ we have $\tilde{z}=\left(\bar{z}_{\tilde{Q}}^{0}, \bar{z}\right) \in \operatorname{cl}$ co $\tilde{\widetilde{Q}}(\bar{t}, \bar{x}, \delta)$, and thus, for every $\delta>0$, there are points $\tilde{z}=\left(z^{0}, z\right) \in \operatorname{co} \tilde{\widetilde{Q}}(\bar{t}, \bar{x}, \delta)$ at a distance as small as we want from $\tilde{z}=\left(\bar{z}^{0}, \bar{z}\right)$. Thus, there is a sequence of points $\tilde{z}_{k}=\left(z_{k}^{0}, z_{k}\right) \in \operatorname{co} Q\left(\bar{t}, \bar{x}, \delta_{k}\right)$ and a sequence of numbers $\delta_{k}>0$ such that $\delta_{k} \rightarrow 0$, $\tilde{z}_{k} \rightarrow \tilde{z}$. In other words, for every integer $k$, there are some pair $\left(t_{k}^{\prime}, x_{k}^{\prime}\right),\left(t_{k}^{\prime \prime}, x_{k}^{\prime \prime}\right)$, corresponding points $\tilde{z}_{k}^{\prime}=\left(z_{k}^{0 \prime}, z_{k}^{\prime}\right) \in Q_{k}\left(t_{k}^{\prime}, x_{k}^{\prime}\right), \quad \tilde{z}_{k}^{\prime \prime}=\left(z_{k}^{0 \prime}, z_{k}^{\prime \prime}\right) \in Q_{k}\left(t_{k}^{\prime \prime}, x_{k}^{\prime \prime}\right)$, points $u_{k}^{\prime}, u_{k}^{\prime \prime} \in E_{m}$, and numbers $\alpha_{k}, 0 \leqq \alpha_{k} \leqq 1$, such that

$$
\begin{array}{ll}
\tilde{z}_{k}=\alpha_{k} \tilde{z}_{k}^{\prime}+\left(1-\alpha_{k}\right) \tilde{z}_{k}^{\prime \prime}, \\
z_{k}^{0}=\alpha_{k} z_{k}^{0 \prime}+\left(1-\alpha_{k}\right) z_{k}^{0 \prime \prime}, & z_{k}=\alpha_{k} z_{k}^{\prime}+\left(1-\alpha_{k}\right) z_{k}^{\prime \prime}, \\
z_{k}^{0^{\prime}} \geqq g\left(t_{k}^{\prime}, x_{k}^{\prime}\right) \phi\left(u_{k}^{\prime}\right)+g_{0}\left(t_{k}^{\prime}, x_{k}^{\prime}\right), & z_{k}^{\prime}=H\left(t_{k}^{\prime}, x_{k}^{\prime}\right) u_{k}^{\prime}+h\left(t_{k}^{\prime}, x_{k}^{\prime}\right), \\
z_{k}^{0 \prime} \geqq g\left(t_{k}^{\prime \prime}, x_{k}^{\prime \prime}\right) \phi\left(u_{k}^{\prime \prime}\right)+g_{0}\left(t_{k}^{\prime \prime}, x_{k}^{\prime \prime}\right), & z_{k}^{\prime \prime}=H\left(t_{k}^{\prime \prime}, x_{k}^{\prime \prime}\right) u_{k}^{\prime \prime}+h\left(t_{k}^{\prime \prime}, x_{k}^{\prime \prime}\right),
\end{array}
$$

and such that $t_{k}^{\prime} \rightarrow \bar{t}, x_{k}^{\prime} \rightarrow \bar{x}, t_{k}^{\prime \prime} \rightarrow \bar{t}, x_{k}^{\prime \prime} \rightarrow \bar{x}, \tilde{z}_{k} \rightarrow \tilde{z}, z_{k}^{0} \rightarrow \bar{z}^{0}, z_{k} \rightarrow \bar{z}$ as $k \rightarrow \infty$. Obviously $g_{0}(t, x)$ is bounded in $N_{\delta}(t, x)$, say $g_{0}(t, x) \geqq-G$ for $G \geqq 0$.

The second relation (4) shows that of the two numbers $z_{k}^{0 \prime}, z_{k}^{0 \prime \prime}$ one must be $\leqq z_{k}^{0}$. It is not restrictive to assume that $z_{k}^{0 \prime} \leqq z_{k}^{0}$ for all $k$. Then the fourth relation (4) yields

$$
z_{k}^{0} \geqq z_{k}^{0 \prime} \geqq g\left(t_{k}^{\prime}, x_{k}^{\prime}\right) \phi\left(u_{k}^{\prime}\right)+g_{0}\left(t_{k}^{\prime}, x_{k}^{\prime}\right) \geqq \mu \phi\left(u_{k}^{\prime}\right)-G,
$$

where $z_{k}^{0} \rightarrow z^{0}$, and hence $\left[z_{k}^{0}\right]$ is a bounded sequence. This shows that $\phi\left(u_{k}^{\prime}\right)$ $\leqq \mu^{-1}\left(G+z_{k}^{0}\right)$, hence $\left[\phi\left(u_{k}\right)\right]$ is a bounded sequence, and finally $\left[u_{k}^{\prime}\right]$ is a bounded sequence because of the property of growth of $\phi$. We can select a subsequence, say still $\left[u_{k}^{\prime}\right]$, which is convergent, say $u_{k}^{\prime} \rightarrow \bar{u}^{\prime} \in E_{m}^{\prime}$ as $k \rightarrow \infty$. The sequence $\left[\alpha_{k}\right]$ is also bounded, hence we can further select a subsequence, say still $\left[\alpha_{k}\right]$, for which $\left[\alpha_{k}\right]$ is also convergent. Thus $u_{k}^{\prime} \rightarrow u^{\prime}, \alpha_{k} \rightarrow \bar{\alpha}$ as $k \rightarrow \infty$. Let $u_{k} \in E_{m}$ be the point $u_{k}=\alpha_{k} u_{k}^{\prime}+\left(1-\alpha_{k}\right) u_{k}^{\prime \prime}$. Then

$$
\begin{aligned}
z_{k}= & \alpha_{k} z_{k}^{\prime}+\left(1-\alpha_{k}\right) z_{k}^{\prime \prime} \\
= & \alpha_{k}\left[H\left(t_{k}^{\prime}, x_{k}^{\prime}\right) u_{k}^{\prime}+h\left(t_{k}^{\prime}, x_{k}^{\prime}\right)\right]+\left(1-\alpha_{k}\right)\left[H\left(t_{k}^{\prime \prime}, x_{k}^{\prime \prime}\right) u_{k}^{\prime \prime}+h\left(t_{k}^{\prime \prime}, x_{k}^{\prime \prime}\right)\right] \\
= & H\left(t_{k}^{\prime \prime}, x_{k}^{\prime \prime}\right)\left[\alpha_{k} u_{k}^{\prime}+\left(1-\alpha_{k}\right) u_{k}^{\prime \prime}\right]+h\left(t_{k}^{\prime \prime}, x_{k}^{\prime \prime}\right) \\
& +\alpha_{k}\left\{\left[H\left(t_{k}^{\prime}, x_{k}^{\prime}\right)-H\left(t_{k}^{\prime \prime}, x_{k}^{\prime \prime}\right)\right] u_{k}^{\prime}+\left[h\left(t_{k}^{\prime}, x_{k}^{\prime}\right)-h\left(t_{k}^{\prime \prime}, x_{k}^{\prime \prime}\right)\right]\right\} \\
= & H\left(t_{k}^{\prime \prime}, x_{k}^{\prime \prime}\right) u_{k}+h\left(t_{k}^{\prime \prime}, x_{k}^{\prime \prime}\right)+\Delta_{k},
\end{aligned}
$$




$$
\begin{aligned}
z_{k}^{0} \geqq & \alpha_{k} z_{k}^{0 \prime}+\left(1-\alpha_{k}\right) z_{k}^{0 \prime \prime} \\
= & \alpha_{k}\left[g\left(t_{k}^{\prime}, x_{k}^{\prime}\right) \phi\left(u_{k}^{\prime}\right)+g_{0}\left(t_{k}^{\prime}, x_{k}^{\prime}\right)\right]+\left(1-\alpha_{k}\right)\left[g\left(t_{k}^{\prime \prime}, x_{k}^{\prime \prime}\right) \phi\left(u_{k}^{\prime \prime}\right)+g_{0}\left(t_{k}^{\prime \prime}, x_{k}^{\prime \prime}\right)\right] \\
= & g\left(t_{k}^{\prime \prime}, x_{k}^{\prime \prime}\right)\left[\alpha_{k} \phi\left(u_{k}^{\prime}\right)+\left(1-\alpha_{k}\right) \phi\left(u_{k}^{\prime \prime}\right)\right]+g_{0}\left(t_{k}^{\prime \prime}, x_{k}^{\prime \prime}\right) \\
& +\alpha_{k}\left\{\left[g\left(t_{k}^{\prime}, x_{k}^{\prime}\right)-g\left(t_{k}^{\prime \prime}, x_{k}^{\prime \prime}\right)\right] \phi\left(u_{k}^{\prime}\right)+\left[g_{0}\left(t_{k}^{\prime}, x_{k}^{\prime}\right)-g_{0}\left(t_{k}^{\prime \prime}, x_{k}^{\prime \prime}\right)\right]\right\} \\
\geqq & g\left(t_{k}^{\prime \prime}, x_{k}^{\prime \prime}\right) \phi\left(u_{k}\right)+g_{0}\left(t_{k}^{\prime \prime}, x_{k}^{\prime \prime}\right)+\Delta_{k}^{0} .
\end{aligned}
$$

Obviously $\Delta_{k} \rightarrow 0, \Delta_{k}^{0} \rightarrow 0, h\left(t_{k}^{\prime \prime}, x_{k}^{\prime \prime}\right) \rightarrow h(\bar{t}, \bar{x}), g_{0}\left(t_{k}^{\prime \prime}, x_{k}^{\prime \prime}\right) \rightarrow g_{0}(\bar{t}, \bar{x})$. Since $g\left(t_{k}^{\prime \prime}, x_{k}^{\prime \prime}\right)$ $\geqq \mu$, we conclude as before that $\left[\phi\left(u_{k}\right)\right]$ is a bounded sequence, and so is $\left[u_{k}\right]$, hence we can further select a convergent subsequence, say still $\left[u_{k}\right]$, with $u_{k} \rightarrow \bar{u}$. Relations (5) yield now as $k \rightarrow \infty$,

$$
\bar{z}=H(\bar{t}, \bar{x}) \bar{u}+h(\bar{t}, \bar{x}), \quad z^{0} \geqq g(\bar{t}, \bar{x}) \phi(\bar{u})+g_{0}(\bar{t}, \bar{x}) .
$$

Thus, $\tilde{z}=\left(z^{0}, z\right) \in \tilde{\tilde{Q}}(\bar{t}, \bar{x})$, and statement (ii) is proved.

REMARK. Here are a few examples of linear problems and corresponding sets $Q(t, x)$ and $\tilde{Q}(t, x)$.

1. Take $m=1, n=2, U=E_{1}$, let $u \in E_{1}$ be the control variable, and take $\phi(u)=1, g=1, g_{0}=0, g_{11}=1, g_{1}=g_{2}=0, g_{21}=t$. Then the sets $Q$ and $\tilde{\tilde{Q}}$ depend on $t,-1 \leqq t \leqq+1$, and

$$
\begin{aligned}
Q(t) & \left.=[z]=\left(z^{1}, z^{2}\right) \mid z^{1}=u, z^{2}=t u,-\infty<u<+\infty\right] \\
& =\left[z=\left(z^{1}, z^{2}\right) \mid z^{2}=t z^{1},-\infty<z^{1}<+\infty\right] \subset E_{2}, \\
\tilde{Q}(t) & =\left[\tilde{z}=\left(z^{0}, z^{1}, z^{2}\right) \mid z^{0} \geqq 1, z^{2}=t z^{1},-\infty<z^{1}<+\infty\right] \subset E_{3} .
\end{aligned}
$$

Each set $Q(t)$ is a straight line in $E_{2}$ of slope $t$, and for each $\delta>0$, the set $Q(0, \delta)$ contains both lines $z^{2}= \pm \delta z^{1}$, and the convex hull of $Q(0, \delta)$ coincides with the whole plane $E_{2}$. Thus $Q(0)$ is the $z^{1}$-axis and $\bigcap_{\delta} \operatorname{clco} Q(0, \delta)$ is the whole $z^{1} z^{2}$ plane. The set $Q(t)$ does not satisfy property $(Q)$ at $t=0$, and the same holds for $\widetilde{\widetilde{Q}}(t)$. Here $\Phi=1$ does not satisfy the growth condition requested in (ii).

2. Take $m=1, n=2, U=E_{1}$, let $u \in E_{1}$, be the control variable, and take $\phi(u)=|u|, \quad g=|t|, g_{0}=0, \quad g_{11}=1, \quad g_{1}=g_{2}=0, \quad g_{21}=t$. Then again the sets $Q$ and $Q \tilde{Q}$ depend on $t$ only, $g \phi=|t u|=\left|z^{2}\right|,-1 \leqq t \leqq 1$.

$$
\begin{aligned}
& Q(t)=\left[z=\left(z^{1}, z^{2}\right) \mid z^{2}=t z^{1},-\infty<z^{1}<+\infty\right] \subset E_{2}, \\
& \tilde{Q}(t)=\left[\tilde{z}=\left(z^{0}, z^{1}, z^{2}\right)\left|z^{0} \geqq\right| z^{2} \mid, z^{2}=t z^{1},-\infty<z^{1}<+\infty\right] \subset E_{3} .
\end{aligned}
$$

As before, the set $Q(t)$ does not satisfy property $(Q)$ at $t=0$. Analogously, for any $\delta>0$, and $-\delta \leqq t \leqq \delta$, we see that

$$
\begin{aligned}
& \tilde{z}^{\prime}=\left(z^{0 \prime}, z^{1 \prime}, z^{2 \prime}\right)=\left(1, \delta^{-1}, 1\right) \in \tilde{\widetilde{Q}}(\delta), \\
& \tilde{z}^{\prime \prime}=\left(z^{0 \prime \prime}, z^{1 \prime \prime}, z^{2 \prime \prime}\right)=\left(1,-\delta^{-1}, 1\right) \in \tilde{\widetilde{Q}}(-\delta),
\end{aligned}
$$


and, for $\alpha=1 / 2$, also

$$
\tilde{z}=\alpha \tilde{z}^{\prime}+(1-\alpha) \tilde{z}^{\prime \prime}=\left(z^{0}, z^{1}, z^{2}\right)=(1,0,1) \in \operatorname{co} \tilde{\tilde{Q}}(0, \delta)
$$

Hence,

$$
\tilde{z}=(1,0,1) \in \cap_{\delta} \operatorname{cl} \operatorname{co} \tilde{\tilde{Q}}(0, \delta), \quad \bar{z}=(1,0,1) \notin \tilde{\tilde{Q}}(0),
$$

and $\tilde{\tilde{Q}}(t)$ does not satisfy property $(Q)$ at $t=0$. Here $g$ does not satisfy the condition $g \geqq \mu>0$ requested in (ii).

3. Take $m=1, n=2, U=E_{1}$, let $u \in E_{1}$ be the control variable, and take $\phi(u)=|\underset{\widetilde{Q}}{u}|, g=1, g_{0}=0, g_{11}=1, g_{1}=g_{2}=0, g_{21}=t$. Then again the sets $Q$ and $\tilde{Q}$ depend on $t$ only, $-1 \leqq t \leqq 1$, and

$$
\begin{aligned}
& Q(t)=\left[z=\left(z^{1}, z^{2}\right) \mid z^{2}=t z^{1},-\infty<z^{1}<+\infty\right] \subset E_{2}, \\
& \tilde{Q}(t)=\left[\tilde{z}=\left(z^{0}, z^{1}, z^{2}\right)\left|z^{0} \geqq\right| z^{1} \mid, z^{2}=t z^{1},-\infty<z^{1}<+\infty\right] \subset E_{3} .
\end{aligned}
$$

As before, $Q(t)$ does not satisfy property $(Q)$, while $\tilde{\tilde{Q}}(t)$ does satisfy property $(Q)$ at every $t$ because of statement (ii).

4. Take $m=n=1, U=E_{1}$, let $u \in E_{1}$ be the control variable, and take $\phi(u)=u^{2}, g=t, g_{0}=0, g_{11}=1, g_{1}=0,0 \leqq t \leqq 1$. Then

$$
\begin{aligned}
& Q(t)=[z \mid z=u,-\infty<u<+\infty] \subset E_{1}, \\
& \tilde{Q}(t)=\left[\tilde{z}=\left(z^{0}, z\right) \mid z^{0} \geqq t u^{2}, z=u,-\infty<u<+\infty\right] \subset E_{2} .
\end{aligned}
$$

Here $Q(t)=U=E_{1}$ for every $t, 0 \leqq t \leqq 1$, and obviously $Q(t)$ satisfies property $(Q)$. On the other hand $\widetilde{\widetilde{Q}}(0)$ is the half plane, $z^{0} \geqq 0,-\infty<z<+\infty$, while $\widetilde{\widetilde{Q}}(t)$ for $t>0$ is the set $Q(t)=\left[z^{0} \geqq t z^{2},-\infty<z<+\infty\right]$. Obviously, $\tilde{\widetilde{Q}}$ satisfies property $(Q)$ at $t=0$ (and at every $t$ as well).

5. Take $m=n=1, U=E_{1}$, let $u \in E_{1}$ be the control variable, and take $\phi(u)=u^{2}, g=t^{3}, g_{0}=0, g_{11}=t, g_{1}=0,0 \leqq t \leqq 1$. Then

$$
\begin{aligned}
& Q(t)=[z \mid z=t u,-\infty<u<+\infty] \subset E_{1}, \\
& \tilde{\tilde{Q}}(t)=\left[\tilde{z}=\left(z^{0}, z\right) \mid z^{0} \geqq t^{3} u^{2}, z=t u,-\infty<u<+\infty\right] \subset E_{2} .
\end{aligned}
$$

Here $Q(0)$ is reduced to the single point $z=0$, while $Q(t)$ for every $t>0$ coincides with $E_{1}$. Thus $Q(t)$ does not satisfy property $(Q)$ at $t=0$. Also $\widetilde{Q}(0)$ $=\left[z^{0} \geqq 0, z=0\right]$ while $\tilde{\widetilde{Q}}(t)$ for $t \neq 0$ is the set $\tilde{\widetilde{Q}}(t)=\left[z^{0} \geqq t z^{2},-\infty<z<+\infty\right]$, and clco $\widetilde{\tilde{Q}}(0, \delta)$ is the entire half plane $\left[z^{0} \geqq 0,-\infty<z<+\infty\right]$. Thus, neither $Q$ nor $\tilde{\tilde{Q}}$ satisfy property $(Q)$ at $t=0$.

We shall denote by $r(t, x)$ the rank of the $n \times m$ matrix $\left(g_{i j}(t, x)\right)$. Then $0 \leqq r(t, x) \leqq \min [m, n]$.

(iii) If all functions $g_{i j}(t, x)$ are continuous and $U=E_{m}$, then $r(\bar{t}, \bar{x}) \leqq$ $\lim \inf r(t, x)$ as $(t, x) \rightarrow(\bar{t}, \bar{x})$. 
The proof is a straightforward consequence of the continuity hypotheses. The statement below shows that a necessary condition for $Q(t, x)$ to satisfy $(Q)$ at $(\bar{t}, \bar{x})$ is that $r(t, x)$ is constant in a neighborhood of $(\bar{t}, \bar{x})$, and this explains why the set $Q$ of Example 4 does not satisfy property $(Q)$. On the other hand, the condition is not sufficient, as the sets $Q$ of Examples 1, 2, 3 show since in these examples $r=1$ is constant.

(iv) If all functions $g_{i j}, g_{i}$ are continuous in $A$ and $U=E_{m}$, then a necessary condition in order that the set $Q(t, x)$ satisfies condition $(Q)$ at $(\bar{t}, \bar{x})$ is that $r(\bar{t}, \bar{x})=\lim r(t, x)$ as $(t, x) \rightarrow(\bar{t}, \bar{x})$ (thus, there is a neighborhood $N_{\delta}(\bar{t}, \bar{x})$ of $(\bar{t}, \bar{x})$ with $r(t, x)=r(\bar{t}, \bar{x})$ for every $(t, x) \in N_{\delta}(\bar{t}, \bar{x})$. If $Q(t, x)$ satisfies condition $(Q)$ in $A$ and $A$ is connected then $r(t, x)$ is a constant.

Proof. Suppose that $r(\bar{t}, \bar{x})=r$ is not the limit of the (integral-valued) function $r(t, x)$ as $(t, x) \rightarrow(\bar{t}, \bar{x})$. Since $r(\bar{t}, \bar{x})=r \leqq \lim$ inf $r(t, x)$ we must have $r(\bar{t}, \bar{x})=r<r+1 \leqq \lim \sup r(t, x)$. There is, therefore, a sequence $\left(t_{k}, x_{k}\right)$, $k=1,2, \cdots$, with $t_{k} \rightarrow \bar{t}, x_{k} \rightarrow \bar{x}$, and $r+1 \leqq r_{k}=r\left(t_{k}, x_{k}\right) \leqq \min [m, n]$. The image of $U=E_{m}$ under the mappings $H\left(t_{k}, x_{k}\right) u+h\left(t_{k}, x_{k}\right)$ and $H(\bar{t}, \bar{x}) u+h(\bar{t}, \bar{x})$ are, therefore, linear manifolds of $E_{n}$, say $Q\left(t_{k}, x_{k}\right)$ of dimensions $r_{k} \geqq r+1$, and $Q(\bar{t}, \bar{x})$ of dimension $r$. The images of $u=0$ on $Q\left(t_{k}, x_{k}\right)$ and $Q(\bar{t}, \bar{x})$ are the points $z_{k}=h\left(t_{k}, x_{k}\right), \bar{z}=h(\bar{t}, \bar{x})$. Let $\eta_{1}, \cdots, \eta_{r}$ be $r$ orthonormal vectors in $E_{n}$ such that

$$
Q(\bar{t}, \bar{x})=\left[z \in E_{n} \mid z=\bar{z}+\xi_{1} \eta_{1}+\cdots+\xi_{r} \eta_{r}, \xi_{1}, \cdots, \xi_{r} \text { real }\right],
$$

and let us complete $\eta_{1}, \cdots, \eta_{r}$ into a system of $n$ orthonormal vectors $\eta_{1}, \cdots, \eta_{r}$, $\eta_{r+1}, \cdots, \eta_{n}$. For every $k$, there are systems of $r_{k}$ orthonormal vectors $\eta_{1 k}^{\prime}, \cdots, \eta_{r_{k}, k}^{\prime}$ of $E_{n}$ such that

$$
Q\left(t_{k}, x_{k}\right)=\left[z \in E \mid z=z_{k}+\xi_{1} \eta_{1 k}^{\prime}+\cdots+\xi_{r_{k}} \eta_{r_{k} k}^{\prime}, \xi_{1}, \cdots, \xi_{r_{k}} \text { real }\right] .
$$

Since $h\left(t_{k}, x_{k}\right) \rightarrow h(\bar{t}, \bar{x}), H\left(t_{k}, x_{k}\right) \rightarrow H(\bar{t}, \bar{x})$, we can select $\eta_{1 k}^{\prime}, \cdots, \eta_{r_{k} k}^{\prime}$ so that, together with $z_{k} \rightarrow \bar{z}$, we have also

$$
\begin{aligned}
\eta_{i k}^{\prime} \cdot \eta_{i} \rightarrow 1, & i=1, \cdots, r, \\
\eta_{j k}^{\prime} \cdot \eta_{i} \rightarrow 0, & j \neq i, j=1, \cdots, r_{k}, \text { as } k \rightarrow \infty .
\end{aligned}
$$

If we take $\xi_{1}=\cdots=\xi_{r}=0, \xi_{r+1}=1, \xi_{r+2}=\cdots=\xi_{r_{k}}=0$, then the point $z_{k}^{\prime}=z_{k}+\eta_{r+1, k} \in Q\left(t_{k}, x_{k}\right)$. It is not restrictive to assume that for all $k$ we have Then

$$
\left|z_{k}-\bar{z}\right|<1 / 4,\left|\eta_{j k} \cdot \eta_{i}\right|<1 / 4 n, \quad j \neq i, i=1, \cdots, r, j=1, \cdots, r_{k} .
$$

$$
\begin{aligned}
\eta_{r+1, k} & =\sum_{i=1}^{n}\left(\eta_{r+1, k} \cdot \eta_{i}\right) \eta_{i}=\left(\sum_{i=1}^{r}+\sum_{i=r+1}^{n}\right)\left(\eta_{r+1, k} \cdot \eta_{i}\right) \eta_{i}=\eta^{\prime}+\eta^{\prime \prime} \\
\left|\eta^{\prime}\right| & =\left|\sum_{i=1}^{r}\left(\eta_{r+1, k} \cdot \eta_{i}\right) \eta_{i}\right| \leqq \sum_{i=1}^{r}\left|\eta_{r+1, k} \cdot \eta_{i}\right| \leqq r(1 / 4 n) \leqq 1 / 4 \\
\left|\eta^{\prime \prime}\right| & =\left|\eta_{r+1, k}-\eta^{\prime}\right| \geqq\left|\eta_{r+1, k}\right|-\left|\eta^{\prime}\right| \geqq 1-1 / 4=3 / 4
\end{aligned}
$$


Finally,

$$
\left|z_{k}^{\prime}-\bar{z}\right|=\left|\left(z_{k}+\eta_{r+1, k}\right)-\bar{z}\right| \leqq\left|\eta_{r+1, k}\right|+\left|z_{k}-\bar{z}\right| \leqq 1+1 / 2=3 / 2,
$$

and, for every $z \in Q(\bar{t}, \bar{x})$, also

$$
\begin{aligned}
\left|z_{k}^{\prime}-z\right| & =\left|\left(z_{k}+\eta_{r+1, k}\right)-\left(\bar{z}+\xi_{1} \eta_{1}+\cdots+\xi_{r} \eta_{r}\right)\right| \\
& =\left|\sum_{i=1}^{n}\left(\eta_{r+1, k} \cdot \eta_{i}\right) \eta_{i}-\sum_{i=1}^{r} \xi_{i} \eta_{i}+\left(z_{k}-\bar{z}\right)\right| \\
& \geqq\left|\sum_{i=1}^{r}\left(\eta_{r+1, k} \cdot \eta_{i}-\xi_{i}\right) \eta_{i}+\sum_{i=r+1}^{n}\left(\eta_{r+1, k} \cdot \eta_{i}\right) \eta_{i}\right|-\left|z_{k}-\bar{z}\right| \\
& \geqq\left|\sum_{i=r+1}^{n}\left(\eta_{r+1, k} \cdot \eta_{i}\right) \eta_{i}\right|-\left|z_{k}-\bar{z}\right|=\left|\eta^{\prime \prime}\right|-\left|z_{k}-\bar{z}\right| \\
& \geqq 3 / 4-1 / 4=1 / 2 .
\end{aligned}
$$

Thus,

$$
\left|z_{k}^{\prime}-\bar{z}\right| \leqq 3 / 2, \quad \operatorname{dist}\left(z_{k}^{\prime}, Q(\bar{t}, \bar{x})\right) \geqq 1 / 2 .
$$

The sequence $\left[z_{k}^{\prime}\right]$ is bounded, hence, it contains a convergent sequence, say still $\left[z_{k}\right]$, with $z_{k}^{\prime} \rightarrow z^{\prime} \in E_{n}$, and

$$
\left|z^{\prime}-\bar{z}\right| \leqq 3 / 2, \quad \operatorname{dist}\left(z^{\prime}, Q(\bar{t}, \bar{x})\right) \geqq 1 / 2 .
$$

Finally, for every $k$ there is a $u_{k} \in U=E_{m}$ such that $z_{k}^{\prime}=H\left(t_{k}, x_{k}\right) u_{k}+h\left(t_{k}, x_{k}\right)$, or $z_{k}^{\prime} \in Q\left(t_{k}, x_{k}\right)$, with $z_{k} \rightarrow z^{\prime}$. Then $z^{\prime} \in \operatorname{cl}$ co $Q(\bar{t}, \bar{x}, \delta)$ for every $\delta>0$, and hence

$$
z^{\prime} \in \bigcap_{\delta} \operatorname{clco} Q(\bar{t}, \bar{x}, \delta), z^{\prime} \notin Q(\bar{t}, \bar{x}) .
$$

We have proved that $Q(t, x)$ does not satisfy property $(Q)$ at $(\bar{t}, \bar{x})$, a contradiction. This proves that, if $Q(t, x)$ satisfies property $(Q)$ at $(\bar{t}, \bar{x})$, then $r(\bar{t}, \bar{x})=\lim r(t, x)$ as $(t, x) \rightarrow(\bar{t}, \bar{x})$. The necessity of the condition is thereby proved.

13. Existence theorems for Lagrange problems with $f$ linear in $u$. We give here a few examples of statements which can be deduced from Existence Theorems I and II when $f$ is linear in $u$.

EXISTENCE THEOREM III. Let us consider the Lagrange problem

$$
\begin{aligned}
& I[x, u]=\int_{t_{1}}^{t_{2}}\left[g(t, x) \phi(u)+g_{0}(t, x)\right] d t=\text { minimum, } \\
& d x^{i} / d t=\sum_{j=1}^{m} g_{i j}(t, x) u^{j}+g_{i}(t, x), \quad i=1, \cdots, n,
\end{aligned}
$$

where $x=\left(x^{1}, \cdots, x^{n}\right) \in E_{n}, u=\left(u^{1}, \cdots, u^{m}\right) \in E_{m}$, and $\phi(u)$ is a continuous nonnegative convex function of $u$. Assume that there is some continuous function 
$\Phi(\zeta), 0 \leqq \zeta<+\infty$, with $\Phi(\zeta) / \zeta \rightarrow+\infty$ as $\zeta \rightarrow+\infty$ and $\phi(u) \geqq \Phi(|u|)$ for every $u \in E_{m}$. Assume that all functions $g(t, x), g_{0}(t, x), g_{i j}(t, x), g_{i}(t, x)$ are continuous in $A=E_{1} \times E_{n}$, and that

$$
g \geqq \mu>0, g_{0} \geqq \mu>0, \quad \sum_{i j}\left|g_{i j}\right| \leqq C g, \sum_{i j}\left|g_{i j}\right|+\sum_{i}\left|g_{i}\right| \leqq C g_{0},
$$

for some constants $\mu>0, C>0$, and all $(t, x) \in A$. Let $\Omega$ be the class of all pairs $x(t), u(t), t_{1} \leqq t \leqq t_{2}, x(t)$ absolutely continuous, $u(t)$ measurable, satisfying (2) a.e., such that $g \phi+g_{0}$ is L-integrable in $\left[t_{1}, t_{2}\right]$, and such that the graph $(t, x(t))$ joins the fixed point $\left(t_{1}=0, x\left(t_{1}\right)=(0, \cdots, 0)\right) \in A$ to a given closed subset $B$ of the half-space $t \geqq 0, x \in E_{n}$ in $A$. If $\Omega$ is not empty, then the Lagrange problem (1), (2) has an optimal solution in $\Omega$.

The functions $\Phi(u)=\phi(|u|)=|u|^{p}, u \in E_{m}, p>1$, as well as $\phi_{c}(u)=\Phi(|u|)$ $=0$ for $|u| \leqq C, \Phi_{c}(u)=\phi(|u|)=|u|^{p}-C^{p}$ for $|u| \geqq C$, certainly satisfy the requirement for $\phi$.

Proof. By Lemmas (i) and (ii) of $\S 12$ the set $\tilde{Q}(t, x)$ is convex for every $(t, x)$ and satisfies condition $(Q)$ in $A$. The set $U=E_{m}$ is fixed, closed, and obviously satisfies condition $(U)$. Also $f_{0}(t, x, u)=g(t, x) \Phi(u)+g_{0}(t, x)$ and hence

$$
f_{0} \geqq \mu \phi(u) \geqq \mu \Phi(|u|), f_{0} \geqq g_{0} \geqq \mu,
$$

where $\mu>0$, and hence both the growth condition for $f_{0}$ and condition (c), of Existence Theorem I of $\S 7$ is satisfied. Now if $A_{0}$ is any compact subset of $A=E_{1} \times E_{n}$, then the continuous functions $g_{i j}, g_{i}$ are bounded in $A_{0}$, say $\left|g_{i j}\right| \leqq C_{0},\left|g_{i}\right| \leqq C_{0}$ (where $C_{0}$ depends on $A_{0}$ ) and

$$
|f|=|H u+h| \leqq|H||u|+|h| \leqq n^{2} C_{0}|u|+n C_{0}
$$

for all $(t, x) \in A_{0}$. Thus condition $(\gamma)$ of Theorem I is also satisfied. Condition (b) is satisfied since the initial point $\left(t_{1}, x\left(t_{1}\right)\right)$ is fixed. Let us prove that condition $\left(\mathrm{a}^{\prime}\right)$ is satisfied. Indeed $\Phi(\zeta) / \zeta \rightarrow+\infty$ as $\zeta \rightarrow+\infty$, hence $\Phi(\zeta) / \zeta \geqq 1$ for all $|\zeta| \geqq D$ and some constant $D \geqq 0$. Then for $|u| \geqq D$ we have $|u| \leqq \Phi(|u|)$, and hence $|u| \leqq D+\Phi(|u|)$ for all $u \in E_{m}$. Now for all $(t, x) \in A=E_{1} \times E_{n}$ and $u \in E_{m}$ we have

$$
\begin{aligned}
|f| & =|H u+h| \leqq|H||u|+|h| \leqq|H|(D+\Phi(|u|))+|h| \\
& =|H| \Phi(|u|)+(D|H|+|h|) \\
& \leqq C g \phi(u)+(D+1) C g_{0} \\
& \leqq C(D+1)\left(g \phi(u)+g_{0}\right)=C(D+1) f_{0} .
\end{aligned}
$$

Thus $f_{0} \geqq C^{-1}(D+1)^{-1}|f|$ for all $(t, x, u) \in E_{1} \times E_{n} \times E_{m}$. All conditions of Theorem I are satisfied, and the Lagrange problem (1), (2) has an optimal solution. 
EXISTENCE THEOREM IV. Let us consider the Lagrange problem

$$
I[x, u]=\int_{t_{1}}^{t_{2}}\left[g(t, x) \phi(u)+g_{0}(t, x)\right] d t=\text { minimum }
$$

with differential equations

$$
d x^{i} / d t=\sum_{j} g_{i j}(t, x) u^{j}+g_{i}(t, x), \quad i=1, \cdots, n,
$$

or

$$
d x / d t=H(t, x) u+h(t, x),
$$

where $x=\left(x^{1}, \cdots, x^{n}\right) \in E_{n}, u=\left(u^{1}, \cdots, u^{m}\right) \in U=E_{m}$, where $H$ is the $n \times m$ matrix $\left(g_{i j}\right)$, where $h$ is the n-vector $\left(g_{i}\right)$, and where $\phi(u)$ is a continuous nonnegative convex function of $u$. Assume that all functions $g(t, x), g_{0}(t, x), g_{i j}(t, x)$, $g_{i}(t, x)$ are continuous in $A=E_{1} \times E_{n}$, and that

$$
\begin{gathered}
g(t, x) \geqq 0, g_{0}(t, x) \geqq-G_{0} \text { for all }(t, x) \in A=E_{1} \times E_{n}, \\
g_{0}(t, x) \geqq \mu>0 \text { for all }(t, x) \in A=E_{1} \times E_{n} \text { with }|t| \geqq D_{0},
\end{gathered}
$$

for some constants $\mu>0, G_{0} \geqq 0, D_{0} \geqq 0$. Assume that the (convex) set

$$
\tilde{\tilde{Q}}(t, x)=\left[\tilde{z}=\left(z^{0}, z\right) \mid z^{0} \geqq g \phi(u)+g_{0}, z=H u+h, u \in U=E_{m}\right] \subset E_{n+1}
$$

satisfies condition $(Q)$ in $A$. Let $\Omega$ be the class of all pairs $x(t), u(t)$, $t_{1} \leqq t \leqq t_{2}, x(t)$ absolutely continuous, $u(t)$ measurable, satisfying (4) a.e., such that $g \phi+g_{0}$ is L-integrable in $\left[t_{1}, t_{2}\right]$, and such that the graph $(t, x(t))$ joins the fixed point $\left(t_{1}=0, x\left(t_{1}\right)=(0, \cdots, 0)\right) \in E_{1} \times E_{n}$ to a given closed subset $B$ of the half-space $t \geqq 0, x \in E_{n}$ in $E_{1} \times E_{n}$ and such that

$$
\int_{t_{1}}^{t_{2}}\left|d x^{i} / d t\right|^{p} d t \leqq N_{i}, \quad i=1, \cdots, n,
$$

for some constants $p>1, N_{i} \geqq 0$. If $\Omega$ is not empty then the Lagrange problem above has an optimal solution in $\Omega$.

The functions $\phi(u)=|u|^{p}, p \geqq 1$, as well as $\phi_{c}(u)=0$ for $|u| \leqq c, \phi(u)$ $=|u|^{p}-c^{p}$ for $|u| \geqq c, p \geqq 1$, all satisfy the requirements above for $\phi$. The requirement $g_{0} \geqq \mu>0$ can be disregarded if $B$ is contained in a slab $\left[0 \leqq t<T, x \in E_{n}\right], T$ finite. Any requirement (6) which is a consequence of a relation $\int_{t_{1}}^{t_{2}}\left(g \Phi+g_{0}\right) d t \leqq N_{0}$ can be disregarded.

Proof. By (i) of $\S 12$ the set $\tilde{Q}(t, x)$ is convex for every $(t, x)$ in $A$. All conditions of Theorem II of $\S 9$ are satisfied, and thus IV is a corollary of II.

REMARK. The requirement concerning $\tilde{\tilde{Q}}(t, x)$ of Theorem IV is certainly satisfied if we assume that

( $\alpha) g(t, x)>0$ for all $(t, x) \in A=E_{1} \times E_{n}$, 
$(\beta)$ there exists a nonnegative convex function $\Phi(\zeta), 0 \leqq \zeta<+\infty$, with $\Phi(\zeta) \rightarrow+\infty$ as $\zeta \rightarrow+\infty$ and $\phi(u) \geqq \Phi(|u|)$ for all $u \in U=E_{m}$.

Indeed, by statements, (i), (ii) of $\S 12$, the convex set $\widetilde{Q}(t, x)$ satisfies property $(Q)$ in $A$.

REMARK. Theorems III and IV can be stated in an analogous form when the special integrand $g \phi+g_{0}$ is replaced by the more general integrand $f_{0}(t, x, u)$ of $\S \S 7$ and 9 .

\section{REFERENCES}

1. L. Cesari, (a) Semicontinuitá e convessitá nel calcolo delle variazioni, Ann. Scuola Norm. Sup. Pisa (3) 18 (1964), 389-423.

(b) Un teorema di esistenza in problemi di controlli ottimi, ibid. (3) 19 (1965), 35-78.

(c) An existence theorem in problems of optimal control, J. Soc. Indust. Appl. Math. Ser. A Control 3 (1965), 7-22.

(d) Existence theorems for optimal solutions in Pontryagin and Lagrange problems, J. Soc. Indust. Appl. Math. Ser. A Control 13 (1965), 475-498.

2. A. F. Filippov, On certain questions in the theory of optimal control, Vestnik Moskov. Univ. Ser. Math. Mech. Astronom. 2 (1959), 25-32 = J. Soc. Indust. Appl. Math. Ser A Control 1 (1962), 76-84.

3. R. V. Gamkrelidze, On sliding optimal states, Dokl. Akad. Nauk SSSR 143 (1962), 1243-1245 = Soviet Math. Dokl. 3, (1962), 559-561.

4. L. Markus and E. B. Lee, Optimal control for nonlinear processes, Arch. Rational Mech. Anal. 8 (1961), 36-58.

5. M. Nagumo, Über die gleichmässige Summierbarkeit und ihre Anwendung auf ein Variations-problem, Japan. J. Math. 6 (1929) 178-182.

6. L. S. Pontryagin, Optimal control processes, Uspehi Mat. Nauk 14 (1959), 3-20; Amer. Math. Soc. Transl. (2) 18 (1961), 321-339.

7. L. S. Pontryagin, V. G. Boltyanskii, R. V. Gamkrelidze, and E. F. Mishchenko, The mathematical theory of optimal processes, Gosudarst, Moscow, 1961 ; English transl., Interscience, New York, 1962; Pergamon Press, New York, 1964.

8. E. O. Roxin, The existence of optimal controls, Michigan Math. J. 9 (1962), 109-119.

9. L. Tonelli, (a) Sugli integrali del calcolo delle variazioni in forma ordinaria, Ann. Scuola Norm. Sup. Pisa (2) 3 (1934), 401-450; Opere Scelte, Vol. 3, Edizioni Cremonese, Rome, 1962, 192-254;

(b) Un teorema di calcolo delle variazioni, Rend. Accad. Naz. Lincei 15 (1932), 417-423; Opere scelte, Vol. 3, 84-91.

(c) Fondamenti di calcolo delle variazioni, Zanichelli, Bologna, 1921-1923, 2 volumes.

10. L. Turner, The direct method in the calculus of variations, Ph.D. Thesis, PurdueUniv., Lafayette, Indiana, 1957.

UNIVERSITY OF MICHIGAN, ANN ARbor, Michigan 